\title{
Coassociative 4-folds with conical singularities
}

\author{
JASON D. LOTAY
}

\begin{abstract}
This article studies the deformation theory of coassociative 4-folds $N$ with conical singularites in a $\mathrm{G}_{2}$ manifold. We describe three moduli spaces: first we consider deformations with the same singularities as $N$, then allow for changes in the singularities and, finally, include variations of the ambient $G_{2}$ structure. We show that the moduli space, in each case, is locally homeomorphic to the kernel of a smooth map between smooth manifolds and determine a lower bound for its expected dimension. Further, by relaxing the condition on the $\mathrm{G}_{2}$ structure, we prove a generic smoothness result for the second and third moduli spaces.
\end{abstract}

\section{Introduction}

Coassociative 4-folds were introduced by Harvey and Lawson in [3] as examples of calibrated 4-dimensional submanifolds of $\mathbb{R}^{7}$ which are linked to the exceptional Lie group $\mathrm{G}_{2}$. Moreover, the coassociative condition is shown to be equivalent to the vanishing of a certain differential 3-form on the submanifold. The definition of coassociative submanifolds, both in $\mathbb{R}^{7}$ and in more general 7-manifolds, is given in $\S 2$.

Manifolds with singularities modelled on cones have been studied by a number of authors, but most relevantly by Lockhart and McOwen [15], in their work on analysis on noncompact manifolds, and by Joyce [6-10], who considered special Lagrangian submanifolds with conical singularities. The latter's work will be discussed in $\S 1.1$. Coassociative 4-folds with conical singularities specifically appear in the work of Kovalev [11] on coassociative fibrations of $\mathrm{G}_{2}$ manifolds. The definition of this class of coassociative 4-folds can be found in $\S 3$.

\subsection{Motivation}

McLean [17] showed that a compact coassociative 4-fold admits a smooth moduli space of deformations with dimension $b_{+}^{2}$. This result was extended by the author [16] to the situation where the coassociative 4-fold is asymptotic to a cone at infinity - a natural progression from the compact case. 
The moduli space is shown to be smooth if the rate at which the 4 -fold converges to the cone at infinity is generic and above a critical value. These studies motivate the work presented here, as a submanifold with conical singularities can be considered as another generalization from the compact to the noncompact category.

Another primary impetus for our research is the work on special Lagrangian $m$-folds with conical singularities by Joyce in the series of papers [6-10]. Special Lagrangian $m$-folds are calibrated submanifolds of CalabiYau manifolds, and can be characterized by the vanishing of differential forms on them. Joyce shows that the moduli space of deformations, for a fixed Calabi-Yau structure, is locally homeomorphic to the kernel of a smooth map between smooth manifolds. Further, he proves a generic smoothness result for the moduli space of deformations in almost CalabiYau manifolds. These results are mimicked in this article, but the proofs are made more difficult essentially because Joyce is able to restrict his attention to analysis using functions, whereas we are forced to deal with differential forms. This may appear to be a minor detail, but it proves to be surprisingly challenging. Another major difference is that we make a detailed calculation of the expected dimension of the moduli space, which requires analytic and topological considerations specific to the coassociative scenario.

We also study coassociative 4 -folds with conical singularities because understanding their deformations should be a useful step towards attempting to prove a 7-dimensional analogue of the SYZ conjecture - a proposed geometric explanation of Mirror Symmetry in String Theory.

\subsection{Summary}

We begin, in $\S 2$, by discussing the notions of $\mathrm{G}_{2}$ structures on 7-manifolds, $\mathrm{G}_{2}$ manifolds and coassociative 4-folds. In $\S 3$, we define a distinguished class of singular manifolds which we call CS manifolds. CS manifolds have conical singularities and their nonsingular part is a noncompact Riemannian manifold. We also define what we mean by tangent cones at conical singularities and CS coassociative 4-folds, that is, coassociative 4-folds with conical singularities.

In order that we may employ various analytic techniques in the course of our study, we choose to use weighted Banach spaces of forms on the nonsingular part of a CS manifold. These spaces are described in $\S 4$. We then focus, in $\S 5$, on a linear, elliptic, first-order differential operator acting between weighted Banach spaces on a 4-dimensional CS manifold. The Fredholm 
and index theory of this operator is discussed using the theory developed in [15].

In $\S 6$, we stratify the types of deformations allowed into three problems, each with an associated nonlinear first-order differential operator whose kernel gives a local description of the moduli space. For Problem 1, we force deformations of the CS coassociative 4 -fold $N$ to have the singularities at the same points as $N$ with the same tangent cones. In Problem 2, we allow the singular points of the deformations, and the tangent cones at these points, to differ from those of $N$. Finally, in Problem 3, we consider deformations of $N$ as in Problem 2 but which now can be coassociative under a deformation of the $G_{2}$ structure within a prescribed family. The main result for each problem, given in $\S 7$, is that the moduli space of deformations near $N$ is homeomorphic to the kernel of a smooth map between smooth manifolds near zero. In each case, the map in question can be considered as a projection from the infinitesimal deformation space onto the obstruction space. Thus, when there are no obstructions, the moduli space is a smooth manifold. Furthermore, using the material in $\S 5$ helps to provide a lower bound on the expected dimension of the moduli space in $\S 8$.

Finally, in $\S 9$, we show that, for a suitable larger category of $\mathrm{G}_{2}$ structures on the ambient 7 -manifold, there is always a family of nearby $\mathrm{G}_{2}$ structures such that the third moduli space is smooth and that, for generic fixed elements of this family, the second moduli space of deformations is smooth as well.

\section{Notes.}

(a) Manifolds are taken to be nonsingular and submanifolds to be embedded, for convenience, unless stated otherwise.

(b) We use the convention that the natural numbers $\mathbb{N}=\{0,1,2, \ldots\}$.

\section{Coassociative 4 -folds}

In this section, we present the basic definitions we need for our study.

The key to defining coassociative 4-folds lies with the introduction of a distinguished 3-form on $\mathbb{R}^{7}$.

Definition 2.1. Let $\left(x_{1}, \ldots, x_{7}\right)$ be coordinates on $\mathbb{R}^{7}$ and write $d \mathbf{x}_{i j \ldots k}$ for the form $d x_{i} \wedge d x_{j} \wedge \cdots \wedge d x_{k}$. Define a 3 -form $\varphi_{0}$ by:

$$
\varphi_{0}=d \mathbf{x}_{123}+d \mathbf{x}_{145}+d \mathbf{x}_{167}+d \mathbf{x}_{246}-d \mathbf{x}_{257}-d \mathbf{x}_{347}-d \mathbf{x}_{356} .
$$


The 4 -form $* \varphi_{0}$, where $\varphi_{0}$ and $* \varphi_{0}$ are related by the Hodge star, is given by:

$$
* \varphi_{0}=d \mathbf{x}_{4567}+d \mathbf{x}_{2367}+d \mathbf{x}_{2345}+d \mathbf{x}_{1357}-d \mathbf{x}_{1346}-d \mathbf{x}_{1256}-d \mathbf{x}_{1247} .
$$

Our choice of expression for $\varphi_{0}$ follows that of [5, Chapter 10]. This form is sometimes known as the $\mathrm{G}_{2} 3$-form because the Lie group $\mathrm{G}_{2}$ is the subgroup of $\mathrm{GL}(7, \mathbb{R})$ preserving $\varphi_{0}$.

Definition 2.2. A 4-dimensional submanifold $N$ of $\mathbb{R}^{7}$ is coassociative if and only if $\left.\varphi_{0}\right|_{N} \equiv 0$ and $\left.* \varphi_{0}\right|_{N}>0$.

This definition is not standard but is equivalent to the usual definition in the language of calibrated geometry by [3, Proposition IV.4.5 and Theorem IV.4.6].

Remark. The condition $\left.\varphi_{0}\right|_{N} \equiv 0$ forces $* \varphi_{0}$ to be a nonvanishing 4-form on $N$. Thus, the positivity of $\left.* \varphi_{0}\right|_{N}$ is equivalent to a choice of orientation on $N$.

So that we may describe coassociative submanifolds of more general 7-manifolds, we make two definitions following [2, p. 7] and [5, p. 243].

Definition 2.3. Let $M$ be an oriented 7-manifold. For each $x \in M$, there exists an orientation preserving isomorphism $\iota_{x}: T_{x} M \rightarrow \mathbb{R}^{7}$. Since dim $\mathrm{G}_{2}=14, \operatorname{dim} \mathrm{GL}_{+}\left(T_{x} M\right)=49$ and $\operatorname{dim} \Lambda^{3} T_{x}^{*} M=35$, the $\mathrm{GL}_{+}\left(T_{x} M\right)$ orbit of $\iota_{x}^{*}\left(\varphi_{0}\right)$ in $\Lambda^{3} T_{x}^{*} M$, denoted by $\Lambda_{+}^{3} T_{x}^{*} M$, is open. A 3 -form $\varphi$ on $M$ is definite if $\left.\varphi\right|_{T_{x} M} \in \Lambda_{+}^{3} T_{x}^{*} M$ for all $x \in M$. Denote the bundle of definite 3 -forms by $\Lambda_{+}^{3} T^{*} M$. It is a bundle with fibre $\mathrm{GL}_{+}(7, \mathbb{R}) / \mathrm{G}_{2}$ which is not a vector subbundle of $\Lambda^{3} T^{*} M$.

Essentially, a definite 3 -form is identified with the $\mathrm{G}_{2} 3$-form on $\mathbb{R}^{7}$ at each point in $M$. Therefore, to each definite 3 -form $\varphi$, we can uniquely associate a 4 -form $* \varphi$ and a metric $g$ on $M$ such that the triple $(\varphi, * \varphi, g)$ corresponds to $\left(\varphi_{0}, * \varphi_{0}, g_{0}\right)$ at each point. This leads us to our next definition.

Definition 2.4. Let $M$ be an oriented 7-manifold, let $\varphi$ be a definite 3 -form on $M$ and let $g$ be the metric associated with $\varphi$. We call $(\varphi, g)$ a $\mathrm{G}_{2}$ structure on $M$. If $\varphi$ is closed (or coclosed) then $(\varphi, g)$ is a closed (or coclosed) $\mathrm{G}_{2}$ structure. A closed and coclosed $\mathrm{G}_{2}$ structure is called torsion-free.

Our choice of notation here agrees with [2]. 


\section{Remarks.}

(a) There is a 1-1 correspondence between pairs $(\varphi, g)$ and principal $\mathrm{G}_{2}$ subbundles of the frame bundle.

(b) By [21, Lemma 11.5], $(\varphi, g)$ is a torsion-free $\mathrm{G}_{2}$ structure if and only if the holonomy of $g$ is contained in $\mathrm{G}_{2}$.

Definition 2.5. Let $M$ be an oriented 7-manifold endowed with a $\mathrm{G}_{2}$ structure $(\varphi, g)$, denoted $(M, \varphi, g)$. We say that $(M, \varphi, g)$ is a $\varphi$-closed, or $\varphi$ coclosed, 7-manifold if $(\varphi, g)$ is a closed, respectively coclosed, $\mathrm{G}_{2}$ structure. If $(\varphi, g)$ is torsion-free, we call $(M, \varphi, g)$ a $\mathrm{G}_{2}$ manifold.

We are now able to extend our definition of coassociative 4-folds.

Definition 2.6. A 4-dimensional submanifold $N$ of $(M, \varphi, g)$ is coassociative if and only if $\left.\varphi\right|_{N} \equiv 0$ and $\left.* \varphi\right|_{N}>0$.

We end this section with a result, [17, cf. Proposition 4.2], that is invaluable in describing the deformation theory of coassociative 4-folds.

Proposition 2.7. Let $N$ be a coassociative 4-fold in $(M, \varphi, g)$. There is an isometric isomorphism between the normal bundle $\nu(N)$ of $N$ in $M$ and $\Lambda_{+}^{2} T^{*} N$ given by $\jmath_{N}:\left.v \mapsto(v \cdot \varphi)\right|_{T N}$.

\section{Conical singularities}

Here we first give the general definition of a manifold with conical singularities, then specialise to coassociative 4-folds. The second definition relies on the introduction of a suitable local coordinate system on the ambient 7-manifold.

\subsection{CS manifolds}

Definition 3.1. Let $N$ be a connected Hausdorff topological space and let $z_{1}, \ldots, z_{s} \in N$ be distinct points. Suppose that $\hat{N}=N \backslash\left\{z_{1}, \ldots, z_{s}\right\}$ has the structure of a (nonsingular) $n$-dimensional Riemannian manifold, with Riemannian metric $g$, compatible with its topology. Then $N$ is a manifold with conical singularities (at $z_{1}, \ldots, z_{s}$ with rate $\lambda$ ) if there exist constants $\epsilon>0$ and $\lambda>1$, a compact $(n-1)$-dimensional Riemannian manifold 
$\left(\Sigma_{i}, h_{i}\right)$, an open set $U_{i} \ni z_{i}$ in $N$ with $U_{i} \cap U_{j}=\emptyset$ for $j \neq i$ and a diffeomorphism $\Psi_{i}:(0, \epsilon) \times \Sigma_{i} \rightarrow U_{i} \backslash\left\{z_{i}\right\} \subseteq \hat{N}$, for $i=1, \ldots, s$, such that

$$
\left|\nabla_{i}^{j}\left(\Psi_{i}^{*}(g)-g_{i}\right)\right|=O\left(r_{i}^{\lambda-1-j}\right) \text { for } j \in \mathbb{N} \text { as } r_{i} \longrightarrow 0,
$$

where $r_{i}$ is the coordinate on $(0, \infty)$ on the cone $C_{i}=(0, \infty) \times \Sigma_{i}, g_{i}=$ $d r_{i}^{2}+r_{i}^{2} h_{i}$ is the conical metric on $C_{i}, \nabla_{i}$ is the Levi-Civita connection derived from $g_{i}$ and $|\cdot|$ is calculated using $g_{i}$.

We call $C_{i}$ the cone at the singularity $z_{i}$ and let the ends $\hat{N}_{\infty}$ of $\hat{N}$ be the disjoint union $\hat{N}_{\infty}=\bigsqcup_{i=1}^{s} U_{i} \backslash\left\{z_{i}\right\}$.

We say that $N$ is $C S$ or a $C S$ manifold (with rate $\lambda$ ) if it is a manifold with conical singularities which have rate $\lambda$ and it is compact as a topological space. In these circumstances it may be written as the disjoint union $N=$ $K \sqcup \bigsqcup_{i=1}^{s} U_{i}$, where $K$ is compact as it is closed in $N$.

The condition $\lambda>1$ guarantees that the metric on $\hat{N}$ genuinely tends to the conical metric on $C_{i}$ near the singularities, as is evident from (3.1). Since $N$ is Hausdorff, the set $U_{i} \backslash\left\{z_{i}\right\}$ is open in $\hat{N}$ for all $i$. Moreover, the condition that the $U_{i}$ are disjoint is easily satisfied since, if $i \neq j, z_{i}$ and $z_{j}$ may be separated by disjoint open sets, and there are only a finite number of singularities.

Remark. If $N$ is a CS manifold, $\hat{N}$ is a noncompact manifold.

Definition 3.2. Let $N$ be a CS manifold. Using the notation of Definition 3.1 , a radius function on $\hat{N}$ is a smooth map $\rho: \hat{N} \rightarrow(0,1]$ such that there exist constants $0<c_{1}<1<c_{2}$ with $c_{1} r_{i}<\Psi_{i}^{*}(\rho)<c_{2} r_{i}$ on $(0, \epsilon) \times \Sigma_{i}$, for all $i$.

If $N$ is CS, we may construct a radius function on $\hat{N}$ as follows. Let $\rho(x)=1$ for all $x \in \hat{N} \backslash \hat{N}_{\infty}$. Define $\rho_{i}: \Psi_{i}\left((0, \epsilon / 2) \times \Sigma_{i}\right) \rightarrow(0,1)$ to be equal to $r_{i} / \epsilon$ for $i=1, \ldots, s$ and then define $\rho$ by interpolating smoothly between its definition on $\hat{N} \backslash \hat{N}_{\infty}$ and $\rho_{i}$ on each of the disjoint sets $\Psi_{i}\left((\epsilon / 2, \epsilon) \times \Sigma_{i}\right)$.

\subsection{CS coassociative 4 -folds}

Let $B(0 ; \eta)$ denote the open ball about 0 in $\mathbb{R}^{7}$ with radius $\eta>0$. We define a preferred choice of local coordinates on a $G_{2}$ manifold near a finite set of points, which is an analogue of the local coordinate system for almost Calabi-Yau manifolds used by Joyce [6, Definition 3.6]. 
Definition 3.3. Let $(M, \varphi, g)$ be a $\mathrm{G}_{2}$ manifold and let $z_{1}, \ldots, z_{s}$ be distinct points in $M$. There exist a constant $\eta \in(0,1)$, an open set $V_{i} \ni z_{i}$ in $M$ with $V_{i} \cap V_{j}=\emptyset$ for $j \neq i$, and a diffeomorphism $\chi_{i}: B(0 ; \eta) \subseteq \mathbb{R}^{7} \rightarrow V_{i}$ with $\chi_{i}(0)=z_{i}$, for $i=1, \ldots, s$, such that $\zeta_{i}=\left.d \chi_{i}\right|_{0}: \mathbb{R}^{7} \rightarrow T_{z_{i}} M$ is an isomorphism identifying the standard $\mathrm{G}_{2}$ structure $\left(\varphi_{0}, g_{0}\right)$ on $\mathbb{R}^{7}$ with the pair $\left(\left.\varphi\right|_{T_{z_{i}} M},\left.g\right|_{T_{z_{i}} M}\right)$. We call the set $\left\{\chi_{i}: B(0 ; \eta) \rightarrow V_{i}: i=1, \ldots, s\right\}$ a $\mathrm{G}_{2}$ coordinate system near $z_{1}, \ldots, z_{s}$.

We say that two $\mathrm{G}_{2}$ coordinate systems near $z_{1}, \ldots, z_{s}$, with maps $\chi_{i}$ and $\tilde{\chi}_{i}$ for $i=1, \ldots, s$ respectively, are equivalent if $\left.d \tilde{\chi}_{i}\right|_{0}=\left.d \chi_{i}\right|_{0}=\zeta_{i}$ for all $i$.

Although the family of $\mathrm{G}_{2}$ coordinate systems near $z_{1}, \ldots, z_{s}$ is clearly infinite-dimensional, there are only finitely many equivalence classes, given by the number of possible sets $\left\{\zeta_{1}, \ldots, \zeta_{s}\right\}$. Moreover, the family of choices for each $\zeta_{i}$ is isomorphic to $\mathrm{G}_{2}$.

Note. Definition 3.3 does not require the $\mathrm{G}_{2}$ structure $(\varphi, g)$ to be torsionfree.

Definition 3.4. Let $(M, \varphi, g)$ be a $\mathrm{G}_{2}$ manifold, let $N \subseteq M$ be compact and connected and let $z_{1}, \ldots, z_{s} \in N$ be distinct points. Let $\left\{\chi_{i}: B(0 ; \eta) \rightarrow\right.$ $\left.V_{i}: i=1, \ldots, s\right\}$ be a $\mathrm{G}_{2}$ coordinate system near $z_{1}, \ldots, z_{s}$ as in Definition 3.3. We say that $N$ is a 4 -fold in $M$ with conical singularities at $z_{1}, \ldots, z_{s}$ with rate $\lambda$, denoted a $C S$ 4-fold, if $\hat{N}=N \backslash\left\{z_{1}, \ldots, z_{s}\right\}$ is a (nonsingular) 4-dimensional submanifold of $M$ and there exist constants $0<\epsilon<\eta$ and $\lambda>1$, a compact 3-dimensional Riemannian submanifold $\left(\Sigma_{i}, h_{i}\right)$ of $\mathcal{S}^{6} \subseteq$ $\mathbb{R}^{7}$, where $h_{i}$ is the restriction of the round metric on $\mathcal{S}^{6}$ to $\Sigma_{i}$, an open set $U_{i} \ni z_{i}$ in $N$ with $U_{i} \subseteq V_{i}$ and a smooth map $\Phi_{i}:(0, \epsilon) \times \Sigma_{i} \rightarrow B(0 ; \eta) \subseteq \mathbb{R}^{7}$, for $i=1, \ldots, s$, such that $\Psi_{i}=\chi_{i} \circ \Phi_{i}:(0, \epsilon) \times \Sigma_{i} \rightarrow U_{i} \backslash\left\{z_{i}\right\}$ is a diffeomorphism, and $\Phi_{i}$ satisfies

$$
\left|\nabla_{i}^{j}\left(\Phi_{i}\left(r_{i}, \sigma_{i}\right)-\iota_{i}\left(r_{i}, \sigma_{i}\right)\right)\right|=O\left(r_{i}^{\lambda-j}\right) \text { for } j \in \mathbb{N} \quad \text { as } r_{i} \longrightarrow 0,
$$

where $\iota_{i}\left(r_{i}, \sigma_{i}\right)=r_{i} \sigma_{i} \in B(0 ; \eta), \nabla_{i}$ is the Levi-Civita connection of the cone metric $g_{i}=d r_{i}^{2}+r_{i}^{2} h_{i}$ on $C_{i}=(0, \infty) \times \Sigma_{i}$ coupled with partial differentiation on $\mathbb{R}^{7}$ and $|\cdot|$ is calculated with respect to $g_{i}$.

We call $C_{i}$ the cone at the singularity $z_{i}$ and $\Sigma_{i}$ the link of the cone $C_{i}$. We may write $N$ as the disjoint union $N=K \sqcup \bigsqcup_{i=1}^{s} U_{i}$, where $K$ is compact.

If $\hat{N}$ is coassociative in $M$, we say that $N$ is a $C S$ coassociative 4 -fold. 
Suppose $N$ is a CS 4 -fold with rate $\lambda$ in $M$ and use the notation of Definition 3.4. The induced metric on $\hat{N},\left.g\right|_{\hat{N}}$, makes $\hat{N}$ into a Riemannian manifold. Moreover, it is clear from (3.2) that the maps $\Psi_{i}$ satisfy (3.1) in Definition 3.1 with the same constant $\lambda$. Thus, $N$ may be considered as a CS manifold with rate $\lambda$.

We now show that, if $\lambda \in(1,2)$, Definition 3.4 is independent of the choice of $\mathrm{G}_{2}$ coordinate system near the singularities, up to equivalence. Suppose we have two equivalent coordinate systems defined using maps $\chi_{i}$ and $\tilde{\chi}_{i}$. These maps must agree up to second order since the zero and firstorder behaviour of each is prescribed, as stated in Definition 3.3. Therefore, the transformed maps $\tilde{\Phi}_{i}$ corresponding to $\tilde{\chi}_{i}$, such that $\tilde{\Psi}_{i}=\tilde{\chi}_{i} \circ \tilde{\Phi}_{i}=\chi_{i}$ ○ $\Phi_{i}=\Psi_{i}$, are defined by $\tilde{\Phi}_{i}=\left(\tilde{\chi}_{i}^{-1} \circ \chi_{i}\right) \circ \Phi_{i}$. Hence

$$
\left|\nabla_{i}^{j}\left(\tilde{\Phi}_{i}\left(r_{i}, \sigma_{i}\right)-\Phi_{i}\left(r_{i}, \sigma_{i}\right)\right)\right|=O\left(r_{i}^{2-j}\right) \quad \text { for } j \in \mathbb{N} \quad \text { as } r_{i} \longrightarrow 0
$$

where $\nabla_{i}$ and $|\cdot|$ are calculated as in Definition 3.4. Thus, if $\lambda<2$, the terms generated by the transformation of the $\mathrm{G}_{2}$ coordinate system neither dominate nor are of equal magnitude to the $O\left(r_{i}^{\lambda-j}\right)$ terms given in (3.2).

We now make a definition which also depends only on equivalence classes of $\mathrm{G}_{2}$ coordinate systems near the singularities.

Definition 3.5. Let $N$ be a CS 4 -fold at $z_{1}, \ldots, z_{s}$ in a $\mathrm{G}_{2}$ manifold $(M, \varphi, g)$. Use the notation of Definitions 3.3 and 3.4. For $i=1, \ldots, s$ define a cone $\hat{C}_{i}$ in $T_{z_{i}} M$ by $\hat{C}_{i}=\left(\zeta_{i} \circ \iota_{i}\right)\left(C_{i}\right)$. We call $\hat{C}_{i}$ the tangent cone at $z_{i}$.

One can show that $\hat{C}_{i}$ is a tangent cone to $N$ at $z_{i}$ in the sense of geometric measure theory (see, for example, [4, p.233]). We also have a straightforward result related to the tangent cones at singular points of CS coassociative 4-folds.

Proposition 3.6. Let $N$ be a $C S$ coassociative 4-fold at $z_{1}, \ldots, z_{s}$ in a $\mathrm{G}_{2}$ manifold $(M, \varphi, g)$. The tangent cones at $z_{1}, \ldots, z_{s}$ are coassociative.

Proof. Use the notation of Definitions 3.3 and 3.4. It is enough to show that $\iota_{i}\left(C_{i}\right)$ is coassociative in $\mathbb{R}^{7}$ for all $i$, since $\zeta_{i}: \mathbb{R}^{7} \rightarrow T_{z_{i}} M$ is an isomorphism identifying $\left(\varphi_{0}, g_{0}\right)$ with $\left(\left.\varphi\right|_{T_{z_{i}} M},\left.g\right|_{T_{z_{i}} M}\right)$. This is equivalent to the condition $\iota_{i}^{*}\left(\varphi_{0}\right) \equiv 0$ for $i=1, \ldots, s$. 
Note that $\left.\varphi\right|_{\hat{N}} \equiv 0$ implies that, for all $i,\left.\varphi\right|_{U_{i} \backslash\left\{z_{i}\right\}} \equiv 0$. Hence, $\Psi_{i}^{*}(\varphi)=$ $\Phi_{i}^{*}\left(\chi_{i}^{*}(\varphi)\right)$ vanishes on $C_{i}$ for all $i$. Using (3.2),

$$
\left|\Phi_{i}^{*}\left(\chi_{i}^{*}(\varphi)\right)-\iota_{i}^{*}\left(\chi_{i}^{*}(\varphi)\right)\right|=O\left(r_{i}^{\lambda-1}\right) \text { as } r_{i} \longrightarrow 0
$$

for all $i$. Moreover,

$$
\left|\iota_{i}^{*}\left(\chi_{i}^{*}(\varphi)\right)-\iota_{i}^{*}\left(\varphi_{0}\right)\right|=O\left(r_{i}\right) \text { as } r_{i} \longrightarrow 0
$$

since

$$
\chi_{i}^{*}(\varphi)=\varphi_{0}+O\left(r_{i}\right) \quad \text { and } \quad\left|\nabla \iota_{i}\right|=O(1) \text { as } r_{i} \longrightarrow 0 .
$$

Therefore, because $\lambda>1$,

$$
\left|\iota_{i}^{*}\left(\varphi_{0}\right)\right| \longrightarrow 0 \text { as } r_{i} \longrightarrow 0
$$

for all $i$. As $T_{r_{i} \sigma_{i}} \iota_{i}\left(C_{i}\right)=T_{\sigma_{i}} \iota_{i}\left(C_{i}\right)$ for all $\left(r_{i}, \sigma_{i}\right) \in C_{i},\left|\iota_{i}^{*}\left(\varphi_{0}\right)\right|$ is independent of $r_{i}$ and thus vanishes for all $i$ as required.

\section{Weighted Banach spaces}

For this section, let $N$ be an $n$-dimensional CS manifold and let $\hat{N}$ be its nonsingular part, as in Definition 3.1. Let $g$ be the metric and let $\nabla$ be the Levi-Civita connection on $\hat{N}$. Moreover, let $\rho$ be a radius function on $\hat{N}$ as in Definition 3.2. We define weighted Banach spaces of forms on $\hat{N}$ as in $[1, \S 1]$, as well as the usual "unweighted" spaces.

We begin with Sobolev spaces.

Definition 4.1. Let $p \geq 1$ and let $k, m \in \mathbb{N}$ with $m \leq n$. The Sobolev space $L_{k}^{p}\left(\Lambda^{m} T^{*} \hat{N}\right)$ is the set of $m$-forms $\xi$ on $\hat{N}$ which are $k$ times weakly differentiable and such that the norm

$$
\|\xi\|_{L_{k}^{p}}=\left(\sum_{j=0}^{k} \int_{\hat{N}}\left|\nabla^{j} \xi\right|^{p} d V_{g}\right)^{1 / p}
$$

is finite. The normed vector space $L_{k}^{p}\left(\Lambda^{m} T^{*} \hat{N}\right)$ is a Banach space for all $p \geq 1$ and $L_{k}^{2}\left(\Lambda^{m} T^{*} \hat{N}\right)$ is a Hilbert space.

We introduce the space of $m$-forms

$$
L_{k, \text { loc }}^{p}\left(\Lambda^{m} T^{*} \hat{N}\right)=\left\{\xi: f \xi \in L_{k}^{p}\left(\Lambda^{m} T^{*} \hat{N}\right) \text { for all } f \in C_{\mathrm{cS}}^{\infty}(\hat{N})\right\}
$$

where $C_{\mathrm{cs}}^{\infty}(\hat{N})$ is the space of smooth functions on $\hat{N}$ with compact support. 
Let $\mu \in \mathbb{R}$. The weighted Sobolev space $L_{k, \mu}^{p}\left(\Lambda^{m} T^{*} \hat{N}\right)$ is the subspace of $L_{k, \text { loc }}^{p}\left(\Lambda^{m} T^{*} \hat{N}\right)$ such that the following norm is finite:

$$
\|\xi\|_{L_{k, \mu}^{p}}=\left(\sum_{j=0}^{k} \int_{\hat{N}}\left|\rho^{j-\mu} \nabla^{j} \xi\right|^{p} \rho^{-n} d V_{g}\right)^{1 / p}
$$

Then $L_{k, \mu}^{p}\left(\Lambda^{m} T^{*} \hat{N}\right)$ is a Banach space and $L_{k, \mu}^{2}\left(\Lambda^{m} T^{*} \hat{N}\right)$ is a Hilbert space.

Note. By comparing the respective norms, it is clear that $L^{p}=L_{0,-n / p}^{p}$. In particular,

$$
L^{2}\left(\Lambda^{m} T^{*} \hat{N}\right)=L_{0,-\frac{n}{2}}^{2}\left(\Lambda^{m} T^{*} \hat{N}\right)
$$

For the following two definitions, of weighted $C^{k}$ and Hölder spaces, we take $C_{\text {loc }}^{k}\left(\Lambda^{m} T^{*} \hat{N}\right)$ to be the vector space of $k$ times continuously differentiable $m$-forms. In the definition of Hölder spaces, we refer to the usual normed vector space $C^{k}\left(\Lambda^{m} T^{*} \hat{N}\right)$ of $k$ times continuously differentiable $m$-forms such that the following norm is finite:

$$
\|\xi\|_{C^{k}}=\sum_{j=0}^{k} \sup _{\hat{N}}\left|\nabla^{j} \xi\right|
$$

Definition 4.2. Let $\mu \in \mathbb{R}$ and let $k, m \in \mathbb{N}$ with $m \leq n$. The weighted $C^{k}$-space $C_{\mu}^{k}\left(\Lambda^{m} T^{*} \hat{N}\right)$ is the subspace of $C_{\text {loc }}^{k}\left(\Lambda^{m} T^{*} \hat{N}\right)$ such that the norm

$$
\|\xi\|_{C_{\mu}^{k}}=\sum_{j=0}^{k} \sup _{\hat{N}}\left|\rho^{j-\mu} \nabla^{j} \xi\right|
$$

is finite. We also define $C_{\mu}^{\infty}\left(\Lambda^{m} T^{*} \hat{N}\right)=\bigcap_{k \geq 0} C_{\mu}^{k}\left(\Lambda^{m} T^{*} \hat{N}\right)$. Then $C_{\mu}^{k}\left(\Lambda^{m}\right.$ $\left.T^{*} \hat{N}\right)$ is a Banach space, but in general $C_{\mu}^{\infty}\left(\Lambda^{m} T^{*} \hat{N}\right)$ is not.

Definition 4.3. Let $V$ be a vector bundle on $\hat{N}$ endowed with Euclidean metrics on its fibres and a connection preserving these metrics. Let $d(x, y)$ be the geodesic distance between points $x, y \in \hat{N}$, let $a \in(0,1)$ and let $k, m \in \mathbb{N}$ 
with $m \leq n$. Let

$$
\begin{aligned}
& H=\left\{(x, y) \in \hat{N} \times \hat{N}: x \neq y, c_{1} \rho(x) \leq \rho(y) \leq c_{2} \rho(x)\right. \text { and } \\
&\text { there exists a geodesic in } \hat{N} \text { of length } d(x, y) \text { from } x \text { to } y\},
\end{aligned}
$$

where $0<c_{1}<1<c_{2}$ are constant. A section $s$ of $V$ is Hölder continuous (with exponent $a$ ) if

$$
[s]^{a}=\sup _{(x, y) \in H} \frac{|s(x)-s(y)|_{V}}{d(x, y)^{a}}<\infty .
$$

We understand the quantity $|s(x)-s(y)|_{V}$ as follows. Given $(x, y) \in H$, there exists a geodesic $\gamma$ of length $d(x, y)$ connecting $x$ and $y$. Parallel translation along $\gamma$ using the connection on $V$ identifies the fibres over $x$ and $y$ and the metrics on them. Thus, with this identification, $|s(x)-s(y)|_{V}$ is well defined.

The Hölder space $C^{k, a}\left(\Lambda^{m} T^{*} \hat{N}\right)$ is the set of $\xi \in C^{k}\left(\Lambda^{m} T^{*} \hat{N}\right)$ such that $\nabla^{k} \xi$ is Hölder continuous (with exponent $a$ ) and the norm

$$
\|\xi\|_{C^{k, a}}=\|\xi\|_{C^{k}}+\left[\nabla^{k} \xi\right]^{a}
$$

is finite. The normed vector space $C^{k, a}\left(\Lambda^{m} T^{*} \hat{N}\right)$ is a Banach space.

We also introduce the notation

$$
\begin{aligned}
& C_{\mathrm{loc}}^{k, a}\left(\Lambda^{m} T^{*} \hat{N}\right) \\
& \quad=\left\{\xi \in C_{\mathrm{loc}}^{k}\left(\Lambda^{m} T^{*} \hat{N}\right): f \xi \in C^{k, a}\left(\Lambda^{m} T^{*} \hat{N}\right) \text { for all } f \in C_{\mathrm{cs}}^{\infty}(\hat{N})\right\} .
\end{aligned}
$$

Let $\mu \in \mathbb{R}$. The weighted Hölder space $C_{\mu}^{k, a}\left(\Lambda^{m} T^{*} \hat{N}\right)$ is the subspace of $C_{\mathrm{loc}}^{k, a}\left(\Lambda^{m} T^{*} \hat{N}\right)$ such that the norm

$$
\|\xi\|_{C_{\mu}^{k, a}}=\|\xi\|_{C_{\mu}^{k}}+[\xi]_{\mu}^{k, a}
$$

is finite, where

$$
[\xi]_{\mu}^{k, a}=\left[\rho^{k+a-\mu} \nabla^{k} \xi\right]^{a} .
$$

Then $C_{\mu}^{k, a}\left(\Lambda^{m} T^{*} \hat{N}\right)$ is a Banach space. It is clear that we have an embedding $C_{\mu}^{k, a}\left(\Lambda^{m} T^{*} \hat{N}\right) \hookrightarrow C_{\mu}^{l}\left(\Lambda^{m} T^{*} \hat{N}\right)$ whenever $l \leq k$.

Remark. The set $H$ in Definition 4.3 is introduced so that $[\xi]_{\mu}^{k, a}$ is well defined. 
We shall need the analogue of the Sobolev embedding theorem for weighted spaces, which is adapted from [15, Lemma 7.2] and [1, Theorem 1.2].

Theorem 4.4 (Weighted Sobolev Embedding Theorem). Let $p, q \geq 1$, $a \in(0,1), \mu, \nu \in \mathbb{R}$ and $k, l, m \in \mathbb{N}$ with $m \leq n$.

(a) If $k \geq l, k-\frac{n}{p} \geq l-\frac{n}{q}$ and either $p \leq q$ and $\mu \geq \nu$ or $p>q$ and $\mu>$ $\nu$, there is a continuous embedding $L_{k, \mu}^{p}\left(\Lambda^{m} T^{*} \hat{N}\right) \hookrightarrow L_{l, \nu}^{q}\left(\Lambda^{m} T^{*} \hat{N}\right)$.

(b) If $k-\frac{n}{p} \geq l+a$, there is a continuous embedding $L_{k, \mu}^{p}\left(\Lambda^{m} T^{*} \hat{N}\right) \hookrightarrow$ $C_{\mu}^{l, a}\left(\Lambda^{m} T^{*} \hat{N}\right)$.

Finally, we shall also require an implicit function theorem for Banach spaces, which follows immediately from [13, Chapter 6, Theorem 2.1].

Theorem 4.5 (Implicit Function Theorem). Let $X$ and $Y$ be Banach spaces and let $W \subseteq X$ be an open neighbourhood of 0 . Let $\mathcal{G}: W \rightarrow Y$ be $a C^{k} \operatorname{map}(k \geq 1)$ such that $\mathcal{G}(0)=0$. Suppose further that $\left.d \mathcal{G}\right|_{0}: X \rightarrow Y$ is surjective with kernel $K$ such that $X=K \oplus A$ for some closed subspace $A$ of $X$. There exist open sets $V \subseteq K$ and $V^{\prime} \subseteq A$, both containing 0 , with $V \times V^{\prime} \subseteq W$, and a unique $C^{k}$ map $\mathcal{V}: V \rightarrow V^{\prime}$ such that

$$
\operatorname{Ker} \mathcal{G} \cap\left(V \times V^{\prime}\right)=\{(x, \mathcal{V}(x)): x \in V\}
$$

in $X=K \oplus A$.

\section{The operator $d+d^{*}$}

In this section we let $N$ be a 4 -dimensional CS manifold and let $\hat{N}$ be as in Definition 3.1. We also let $\rho$ be a radius function on $\hat{N}$ as in Definition 3.2. An essential part of our study is the use of the Fredholm and index theory for the elliptic operator $d+d^{*}$ acting from $\Lambda_{+}^{2} T^{*} \hat{N} \oplus \Lambda^{4} T^{*} \hat{N}$ to $\Lambda^{3} T^{*} \hat{N}$, where we identify the image of $d$ and $d^{*}$ acting on self-dual 2 -forms. We therefore consider

$$
d+d^{*}: L_{k+1, \mu}^{p}\left(\Lambda_{+}^{2} T^{*} \hat{N} \oplus \Lambda^{4} T^{*} \hat{M}\right) \longrightarrow L_{k, \mu-1}^{p}\left(\Lambda^{3} T^{*} \hat{N}\right),
$$

where $p \geq 2, k \in \mathbb{N}$ and $\mu \in \mathbb{R}$.

\subsection{Fredholm theory}

Our first result follows from [15, Theorems 1.1 and 6.1]. 
Proposition 5.1. There exists a countable discrete set $\mathcal{D} \subseteq \mathbb{R}$ such that (5.1) is Fredholm if and only if $\mu \notin \mathcal{D}$.

Moreover, we can give an explicit description of $\mathcal{D}$ by similar arguments to [18, Lemma 6.1.2] and [16, pp. 13-14], which are both for asymptotically conical (AC) manifolds. The argument is long and technical, but the idea is that to use the formalism of [15] one needs to consider manifolds with cylindrical ends, so we associate to our operator (5.1) another which acts on cylinders. We then push down to a map acting on forms on the crosssections of the cylinders and it is the Fredholm theory of this map which determines the set $\mathcal{D}$.

Recall the notation of Definition 3.1. If $\left(r_{i}, \sigma_{i}\right)$ are the coordinates on $(0, \epsilon) \times \Sigma_{i}$, let $t_{i}=-\log r_{i}$, recalling that $\epsilon<\eta<1$. Thus $\left(t_{i}, \sigma_{i}\right)$ are cylindrical coordinates on $(T, \infty) \times \Sigma_{i}$, where $T=-\log \epsilon>0$. Transform the metric on $\hat{N}$ to a conformally equivalent metric which is asymptotically cylindrical on the ends $\hat{N}_{\infty} \cong \bigsqcup_{i=1}^{s}(T, \infty) \times \Sigma_{i}$ of $\hat{N}$; that is, the metric on $\hat{N}$ is asymptotic to $d t_{i}^{2}+h_{i}$ on the $i$ th end. With respect to this new metric, the asymptotically cylindrical operator corresponding to $d+d^{*}$ acts as

$$
\rho^{-m} d \rho^{m}+\rho^{-m+2} d^{*} \rho^{m}
$$

on $m$-forms on $\hat{N}$. We refer the interested reader to $[14$, Proposition and Definition 4.4] to explain the introduction of these powers of $\rho$. Since $\rho$ is asymptotic to $r_{i}=e^{-t_{i}}$, we have a cylindrical operator $\left(d+d^{*}\right)_{\infty}$ associated to $d+d^{*}$ which acts on $m$-forms on each component of $\hat{N}_{\infty} \cong \bigsqcup_{i=1}^{s}(T, \infty) \times$ $\Sigma_{i}$ by

$$
\left(d+d^{*}\right)_{\infty}=e^{m t_{i}}\left(d+e^{-2 t_{i}} d^{*}\right) e^{-m t_{i}}
$$

Notice that an $m$-form $\xi$ on $\hat{N}_{\infty}$ can be written on the $i$ th end as

$$
\xi\left(t_{i}, \sigma_{i}\right)=\Omega_{i}\left(t_{i}, \sigma_{i}\right)+d t \wedge \omega_{i}\left(t_{i}, \sigma_{i}\right)
$$

where for each $t_{i} \in(T, \infty), \Omega_{i}\left(t_{i}, \sigma_{i}\right)$ and $\omega_{i}\left(t_{i}, \sigma_{i}\right)$ are $m$ - and $(m-1)$-forms on $\Sigma_{i}$ respectively. Thus, if $p_{i}:(0, \infty) \times \Sigma_{i} \rightarrow \Sigma_{i}$ is the natural projection map,

$$
\Lambda^{m} T^{*} \hat{N}_{\infty} \cong \bigsqcup_{i=1}^{s}\left(p_{i}^{*}\left(\Lambda^{m} T^{*} \Sigma_{i}\right) \oplus p_{i}^{*}\left(\Lambda^{m-1} T^{*} \Sigma_{i}\right)\right)
$$


Hence, $\left(d+d^{*}\right)_{\infty}$ maps sections of $p_{i}^{*}\left(\Lambda^{2} T^{*} \Sigma_{i}\right) \oplus p_{i}^{*}\left(\Lambda^{\text {odd }} T^{*} \Sigma_{i}\right)$ to sections of $p_{i}^{*}\left(\Lambda^{\text {odd }} T^{*} \Sigma_{i}\right) \oplus p_{i}^{*}\left(\Lambda^{\text {even }} T^{*} \Sigma_{i}\right)$. Moreover, this action is given by:

$$
\begin{aligned}
& \left(d+d^{*}\right)_{\infty}\left(\begin{array}{c}
\alpha_{i}\left(t_{i}, \sigma_{i}\right) \\
\gamma_{i}\left(t_{i}, \sigma_{i}\right)+\beta_{i}\left(t_{i}, \sigma_{i}\right)
\end{array}\right) \\
& =\left(\begin{array}{cc}
d_{i}+d_{i}^{*} & -\frac{\partial}{\partial t_{i}}+3-m \\
\frac{\partial}{\partial t_{i}}-m & -\left(d_{i}+d_{i}^{*}\right)
\end{array}\right)\left(\begin{array}{c}
\alpha_{i}\left(t_{i}, \sigma_{i}\right) \\
\gamma_{i}\left(t_{i}, \sigma_{i}\right)+\beta_{i}\left(t_{i}, \sigma_{i}\right)
\end{array}\right)
\end{aligned}
$$

where $d_{i}$ and $d_{i}^{*}$ are the exterior derivative and its adjoint on $\Sigma_{i}$, and $m$ denotes the operator which multiplies $m$-forms by a factor $m$.

However, we wish to restrict attention to forms on $\Sigma_{i}$ which correspond, via $p_{i}^{*}$, to self-dual 2 -forms on $\hat{N}_{\infty}$ rather than general 2 -forms. So, we define $S_{i} \subseteq \Lambda^{2} T^{*} \Sigma_{i} \oplus \Lambda^{\text {odd }} T^{*} \Sigma_{i}$ by

$$
S_{i}=\left\{\left(\alpha_{i}, *_{i} \alpha_{i}+\beta_{i}\right): \alpha_{i} \in \Lambda^{2} T^{*} \Sigma_{i}, \beta_{i} \in \Lambda^{3} T^{*} \Sigma_{i}\right\}
$$

where $*_{i}$ is the Hodge star on $\Sigma_{i}$. Then $\bigsqcup_{i=1}^{s} p_{i}^{*}\left(S_{i}\right) \cong \Lambda_{+}^{2} T^{*} \hat{N}_{\infty} \oplus \Lambda^{4} T^{*} \hat{N}_{\infty}$.

For $w \in \mathbb{C}$, define a map $\left(d+d^{*}\right)_{\infty}(w)$ which acts on sections of $S_{i} \otimes \mathbb{C}$ by:

$$
\left(d+d^{*}\right)_{\infty}(w)=\left(\begin{array}{cc}
d_{i}+d_{i}^{*} & w+3-m \\
-w-m & -\left(d_{i}+d_{i}^{*}\right)
\end{array}\right)
$$

Notice that we have formally substituted $w$ for $-\frac{\partial}{\partial t_{i}}$ in $(5.2)$.

Let

$$
T_{i}=\left\{\left(*_{i} \alpha_{i}+\beta_{i}, \alpha_{i}\right): \alpha_{i} \in \Lambda^{2} T^{*} \Sigma_{i}, \beta_{i} \in \Lambda^{3} T^{*} \Sigma_{i}\right\},
$$

so that $\bigsqcup_{i=1}^{s} p_{i}^{*}\left(T_{i}\right) \cong \Lambda^{3} T^{*} \hat{N}_{\infty}$. Define $\mathcal{C} \subseteq \mathbb{C}$ as the set of $w$ for which

$$
\left(d+d^{*}\right)_{\infty}(w): L_{k+1}^{p}\left(S_{i} \otimes \mathbb{C}\right) \rightarrow L_{k}^{p}\left(T_{i} \otimes \mathbb{C}\right)
$$

where we include a projection to $T_{i}$, is not an isomorphism for any $i$. By the proof of [15, Theorem 1.1], D $=\{\operatorname{Re} w: w \in \mathcal{C}\}$. By [18, Lemma 6.1.13], the corresponding sets $\mathcal{C}\left(\Delta^{m}\right)$, where $\Delta^{m}$ is the Laplacian on $m$-forms, are all real for an AC manifold. Since the same will be true for the CS case, we deduce that $\mathcal{C} \subseteq \mathbb{R}$. Hence $\mathcal{C}=\mathcal{D}$.

The symbol, hence the index ind ${ }_{w}$, of $\left(d+d^{*}\right)_{\infty}(w)$ is independent of $w$. Furthermore, $\left(d+d^{*}\right)_{\infty}(w)$ is an isomorphism for generic values of $w$ since 
$\mathcal{D}$ is countable and discrete. Therefore $\operatorname{ind}_{w}=0$ for all $w \in \mathbb{C}$; that is,

$$
\operatorname{dim} \operatorname{Ker}\left(d+d^{*}\right)_{\infty}(w)=\operatorname{dim} \operatorname{Coker}\left(d+d^{*}\right)_{\infty}(w)
$$

so that (5.3) is not an isomorphism precisely when it is not injective.

The condition $\left(d+d^{*}\right)_{\infty}(w)=0$, using (5.3), corresponds to the existence of $\alpha_{i} \in C^{\infty}\left(\Lambda^{2} T^{*} \Sigma_{i}\right)$ and $\beta_{i} \in C^{\infty}\left(\Lambda^{3} T^{*} \Sigma_{i}\right)$, for some $i$, satisfying

$$
d_{i} \alpha_{i}=-w \beta_{i} \quad \text { and } \quad d_{i} *_{i} \alpha_{i}+d_{i}^{*} \beta_{i}=-(w+2) \alpha_{i}
$$

Before we state our proposition, we make a few observations concerning $\mathcal{D}$.

\section{Notes.}

(a) Clearly, (5.4) implies that $d_{i} d_{i}^{*} \beta_{i}=\Delta_{i} \beta_{i}=w(w+2) \beta_{i}$. Since eigenvalues of the Laplacian on $\Sigma_{i}$ must necessarily be positive, $\beta_{i}=0$ if $w \in(-2,0)$.

(b) If $w=0$ and we take $\alpha_{i}=0,(5.4)$ forces $\beta_{i}$ to be coclosed. As there are nontrivial coclosed 3 -forms on $\Sigma_{i},\left(d+d^{*}\right)_{\infty}(0)$ is not injective, so $0 \in \mathcal{D}$.

(c) Suppose that $w=-2 \in \mathcal{D}$. Then (5.4) gives $\left[\beta_{i}\right]=0$ in $H_{\mathrm{dR}}^{3}\left(\Sigma_{i}\right)$. Using (a), $\beta_{i}$ is harmonic so, by Hodge theory, $\beta_{i}=0$. Therefore $-2 \in \mathcal{D}$ if and only if there exists a nonzero closed and coclosed 2 -form on $\Sigma_{i}$ for some $i$.

Proposition 5.2. Use the notation of Definition 3.1 and the discussion above. For $i=1, \ldots, s$ let

$$
\begin{gathered}
D(\mu, i)=\left\{\left(\alpha_{i}, \beta_{i}\right) \in C^{\infty}\left(\Lambda^{2} T^{*} \Sigma_{i} \oplus \Lambda^{3} T^{*} \Sigma_{i}\right): d_{i} \alpha_{i}=-\mu \beta_{i},\right. \\
\left.d_{i} *_{i} \alpha_{i}+d_{i}^{*} \beta_{i}=-(\mu+2) \alpha_{i}\right\} .
\end{gathered}
$$

The set $\mathcal{D}$ of real numbers $\mu$ such that (5.1) is not Fredholm is given by:

$$
\mathcal{D}=\bigcup_{i=1}^{s}\{\mu \in \mathbb{R}: D(\mu, i) \neq 0\}
$$


Remark. A perhaps more illuminating way to characterise $D(\mu, i)$ is by:

$$
\begin{aligned}
\left(\alpha_{i}, \beta_{i}\right) \in D(\mu, i) & \Longleftrightarrow \xi_{i}=\left(r_{i}^{\mu+2} \alpha_{i}+r_{i}^{\mu+1} d r_{i} \wedge * \alpha_{i}, r_{i}^{\mu+3} d r_{i} \wedge \beta_{i}\right) \\
& \text { is an } O\left(r_{i}^{\mu}\right) \text { solution of }\left(d+d^{*}\right) \xi_{i}=0 \text { on } C_{i} \text { as } r_{i} \longrightarrow 0,
\end{aligned}
$$

using the notation of Definition 3.1.

Lockhart and McOwen [15, §10] study the Laplacian on $m$-forms on a manifold with a conical singularity. From this work, which can easily be extended to manifolds with more than one singularity, we can make an important observation about the set $\mathcal{D}$.

Proposition 5.3. In the notation of Proposition 5.2, $\mathcal{D} \cap(-2,-1]=\emptyset$.

Proof. Let $\mu \in(-2,-1]$ and let

$$
\Delta^{m}: L_{k+1, \mu}^{p}\left(\Lambda^{m} T^{*} \hat{N}\right) \longrightarrow L_{k-1, \mu-2}^{p}\left(\Lambda^{m} T^{*} \hat{N}\right)
$$

be the Laplacian on $m$-forms and denote the set of $\mu$ such that it is not Fredholm by $\mathcal{D}\left(\Delta^{m}\right)$. Since $\mu>-2$ and $p \geq 2$, we see that $L_{k+1, \mu}^{p} \hookrightarrow L_{0,-2}^{2}=L^{2}$ by Theorem 4.4 and (4.1).

Applying [15, Theorem 10.2] to $\Delta^{2}$ and $\Delta^{4}$, we see that

$$
\mathcal{D}\left(\Delta^{2}\right) \cap(-2,-1]=\mathcal{D}\left(\Delta^{4}\right) \cap(-2,-1]=\emptyset .
$$

Note that our rate $\mu$ is related to the weighting factor $\nu$ in $[15, \S 10]$ by $\mu=$ $-\nu-2$. Since it is clear that $\mathcal{D} \subseteq\left(\mathcal{D}\left(\Delta^{2}\right) \cup \mathcal{D}\left(\Delta^{4}\right)\right)$, the result follows.

\subsection{Index theory}

We begin with some definitions following [15].

Definition 5.4. Use the notation of $\S 5.1$. Let $\mu \in \mathcal{D}$. Define $d(\mu)$ to be the dimension of the vector space of solutions of $\left(d+d^{*}\right)_{\infty} \xi=0$ of the form

$$
\xi\left(t_{i}, \sigma_{i}\right)=e^{-\mu t_{i}} q_{i}\left(t_{i}, \sigma_{i}\right)
$$

on $(T, \infty) \times \Sigma_{i}$, for $i=1, \ldots, s$, where $q_{i}\left(t_{i}, \sigma_{i}\right)$ is a polynomial in $t_{i}$ with coefficients in $C^{\infty}\left(S_{i} \otimes \mathbb{C}\right)$.

The next result is immediate from [15, Theorem 1.2]. 
Theorem 5.5. Use the notation of Proposition 5.1 and Definition 5.4. Let $\lambda, \lambda^{\prime} \notin \mathcal{D}$ with $\lambda^{\prime} \leq \lambda$. If $\operatorname{ind}_{\mu}\left(d+d^{*}\right)$ denotes the index of (5.1) for $\mu \notin \mathcal{D}$,

$$
\operatorname{ind}_{\lambda^{\prime}}\left(d+d^{*}\right)-\operatorname{ind}_{\lambda}\left(d+d^{*}\right)=\sum_{\mu \in \mathcal{D} \cap\left(\lambda^{\prime}, \lambda\right)} d(\mu) .
$$

We make an observation which shall be useful in later sections.

Proposition 5.6. Let $\lambda, \lambda^{\prime} \in \mathbb{R}$ such that $\lambda^{\prime} \leq \lambda$ and $\left[\lambda^{\prime}, \lambda\right] \cap \mathcal{D}=\emptyset$. The kernels, and cokernels, of (5.1) when $\mu=\lambda$ and $\mu=\lambda^{\prime}$ are equal.

Proof. Denote the dimensions of the kernel and cokernel of (5.1), for $\mu \notin \mathcal{D}$, by $k(\mu)$ and $c(\mu)$ respectively. Since $\left[\lambda^{\prime}, \lambda\right] \cap \mathcal{D}=\emptyset, k(\lambda)-c(\lambda)=k\left(\lambda^{\prime}\right)-$ $c\left(\lambda^{\prime}\right)$, so

$$
k(\lambda)-k\left(\lambda^{\prime}\right)=c(\lambda)-c\left(\lambda^{\prime}\right) .
$$

We know that $k(\lambda) \leq k\left(\lambda^{\prime}\right)$ because $L_{k+1, \lambda}^{p} \hookrightarrow L_{k+1, \lambda^{\prime}}^{p}$ by Theorem 4.4 as $\lambda \geq \lambda^{\prime}$. Similarly, since $c(\mu)$ is equal to the dimension of the kernel of the formal adjoint operator acting on a Sobolev space with weight $-3-\mu=$ $-4-(\mu-1), c(\lambda) \geq c\left(\lambda^{\prime}\right)$. Noting that the right-hand side of (5.5) is nonnegative and the left-hand side is less than or equal to zero, we conclude that both must be zero. The result follows from the fact that the kernel of $d+d^{*}$ in $L_{k+1, \lambda}^{p}$ is contained in the kernel of $d+d^{*}$ in $L_{k+1, \lambda^{\prime}}^{p}$, and vice versa for the cokernels.

We conclude by relating $d(\mu)$ and $D(\mu, i)$ in a result analogous to [16, Proposition 5.4], and which can be proved in exactly the same manner.

Proposition 5.7. Using the notation of Proposition 5.2 and Definition 5.4, $d(\mu)=\sum_{i=1}^{s} \operatorname{dim} D(\mu, i)$ for $\mu \in \mathcal{D}$.

\section{The deformation problems}

We have a common notation for the next three sections. Let $N$ be a CS coassociative 4 -fold at $z_{1}, \ldots, z_{s}$ with rate $\lambda$ in a $\mathrm{G}_{2}$ manifold $(M, \varphi, g)$. Suppose $\lambda \in(1,2) \backslash \mathcal{D}$, where $\mathcal{D}$ is given by Proposition 5.2, and the cone at $z_{i}$ is $C_{i}$ with link $\Sigma_{i}$. We shall then use the notation of Definitions 3.4 and 3.5. In particular, we let $\left\{\chi_{i}: B(0 ; \eta) \rightarrow V_{i}: i=1, \ldots, s\right\}$, with $\left.d \chi_{i}\right|_{0}=\zeta_{i}$ for all $i$, be the $\mathrm{G}_{2}$ coordinate system near $z_{1}, \ldots, z_{s}$ used to define $N$ and we let $\hat{C}_{i}$ be the tangent cone at $z_{i}$. We also write $N=K \sqcup \bigsqcup_{i=1}^{s} U_{i}$, where 
$K$ is compact and $U_{i}$ is an open set in $N$ containing $z_{i}$. Moreover, we let $\rho$ be a radius function on $\hat{N}$ as in Definition 3.2 and choose the smooth maps $\Phi_{i}:(0, \epsilon) \times \Sigma_{i} \rightarrow B(0 ; \eta)$ uniquely by imposing the condition

$$
\Phi_{i}\left(r_{i}, \sigma_{i}\right)-\iota_{i}\left(r_{i}, \sigma_{i}\right) \in\left(T_{r_{i} \sigma_{i}} \iota_{i}\left(C_{i}\right)\right)^{\perp} \text { for all }\left(r_{i}, \sigma_{i}\right) \in(0, \epsilon) \times \Sigma_{i},
$$

where $\iota_{i}\left(r_{i}, \sigma_{i}\right)=r_{i} \sigma_{i}$ is the inclusion map on $C_{i}$. The condition on $\Phi_{i}$ can always be achieved by making $\epsilon$ and the sets $U_{i}$ smaller. We shall endeavour to remind the reader when we use specific elements of this notation.

In this section, we consider deformations of $N$ which are CS coassociative 4 -folds at $s$ points with rate $\lambda$ in $(M, \varphi, g)$ with the same cones at the singularities as $N$, but the singularities need not be at the same points, nor have identical tangent cone. We also, eventually, allow variations in the $\mathrm{G}_{2}$ structure on the ambient 7-manifold $M$, and include deformations of $N$ which may be coassociative with respect to a nearby $\mathrm{G}_{2}$ structure.

We tackle this deformation theory by splitting it into three moduli space problems, each one allowing for more possible deformations of $N$. We do this to help the reader because each time the notation becomes more cluttered and the analysis more complicated, but most of the important ideas are clear from the solution of the first problem.

\subsection{Problem 1: fixed singularities and $\mathrm{G}_{2}$ structure}

The first problem we study is where the deformations of $N$ have identical singular points to $N$ with the same rate, cones and tangent cones, and the $\mathrm{G}_{2}$ structure on $M$ is fixed. We give a formal definition for this moduli space.

Definition 6.1. The moduli space of deformations $\mathcal{M}_{1}(N, \lambda)$ for Problem 1 is the set of $N^{\prime}$ in $(M, \varphi, g)$ which are CS coassociative 4-folds at $z_{1}, \ldots, z_{s}$ with rate $\lambda$, having cone $C_{i}$ and tangent cone $\hat{C}_{i}$ at $z_{i}$ for all $i$, such that there exists a diffeomorphism $h: M \rightarrow M$, isotopic to the identity, such that $h\left(z_{i}\right)=z_{i}$ for $i=1, \ldots, s,\left.h\right|_{N}: N \rightarrow N^{\prime}$ is a homeomorphism and $\left.h\right|_{\hat{N}}: \hat{N} \rightarrow$ $N^{\prime} \backslash\left\{z_{1}, \ldots, z_{s}\right\}$ is a diffeomorphism.

We begin our formulation of a local description of $\mathcal{M}_{1}(N, \lambda)$ with a result which is immediate from the proof of [12, Chapter IV, Theorem 9] since $M$ is a Riemannian manifold.

Theorem 6.2. Let $P$ be a closed embedded submanifold of $M$. There exist an open subset $V$ of the normal bundle $\nu(P)$ of $P$ in $M$, containing the zero 
section, and an open set $S$ in $M$ containing $P$, such that the exponential map $\left.\exp \right|_{V}: V \rightarrow S$ is a diffeomorphism.

Note. The proof of this result relies entirely on the observation that $\left.\exp \right|_{\nu(P)}$ is a local isomorphism upon the zero section.

This information provides us with a useful corollary to Theorem 6.2.

Corollary 6.3. Let $P_{i}=\iota_{i}\left((0, \epsilon) \times \Sigma_{i}\right), Q_{i}=\Phi_{i}\left((0, \epsilon) \times \Sigma_{i}\right)$ and define $n_{i}: \nu\left(P_{i}\right) \rightarrow \mathbb{R}^{7}$ by $n_{i}\left(r_{i} \sigma_{i}, v\right)=v+\Phi_{i}\left(r_{i}, \sigma_{i}\right)$. For all $i$, there exist an open subset $\hat{V}_{i}$ of $\nu\left(P_{i}\right)$ in $\mathbb{R}^{7}$, containing the zero section, and an open set $\hat{S}_{i}$ in $B(0 ; \eta) \subseteq \mathbb{R}^{7}$ containing $Q_{i}$ such that $\left.n_{i}\right|_{\hat{V}_{i}}: \hat{V}_{i} \rightarrow \hat{S}_{i}$ is a diffeomorphism. Moreover, $\hat{V}_{i}$ and $\hat{S}_{i}$ can be chosen such that $P_{i} \subseteq \hat{S}_{i}$ and $\hat{V}_{i}$ is an open set in $C_{1}^{1}$.

Proof. Note that $n_{i}$ takes the zero section of $\nu\left(P_{i}\right)$ to $Q_{i}$. By the definition of $\Phi_{i}, n_{i}$ is a local isomorphism upon the zero section. Thus, the proof of Theorem 6.2 gives open sets $\hat{V}_{i}$ and $\hat{S}_{i}$ such that $\left.n_{i}\right|_{\hat{V}_{i}}: \hat{V}_{i} \rightarrow \hat{S}_{i}$ is a diffeomorphism. We can ensure that $\hat{S}_{i}$ lies in $B(0 ; \eta)$ by making $\hat{V}_{i}$ smaller if necessary.

Furthermore, since $\Phi_{i}-\iota_{i}$ is orthogonal to $P_{i}$ by $(6.1)$, it can be identified with a small section of $\nu\left(P_{i}\right)$. Hence $P_{i}$ lies in $\hat{S}_{i}$ as long as $\hat{S}_{i}$ grows at $O\left(r_{i}\right)$ as $r_{i} \rightarrow 0$. As we can form $\hat{S}_{i}$ and $\hat{V}_{i}$ in a translation equivariant way because we are working on a portion of the cone $C_{i}$, we can construct our sets with this decay rate as $r_{i} \rightarrow 0$ and such that they do not collapse as $r_{i} \rightarrow \epsilon$. Thus, we can ensure that $\hat{V}_{i}$ is an open set in $C_{1}^{1}$ as claimed.

The reason for using Corollary 6.3, rather than simply considering exponential normal deformations as given by Theorem 6.2 , is that it gives us control of the behaviour of the sets $\hat{V}_{i}$ and $\hat{S}_{i}$ near 0 . This is essential in making our analysis valid.

By Proposition 2.7 and Theorem 6.2, we can think of nearby deformations of $N$ as graphs of small self-dual 2-forms. The next proposition gives a formal backing to this concept.

Proposition 6.4. There exist an open set $\hat{U} \subseteq \Lambda_{+}^{2} T^{*} \hat{N}$ containing the zero section, an open set $\hat{T} \subseteq M$ containing $\hat{N}$ and a diffeomorphism $\delta: \hat{U} \rightarrow \hat{T}$ which takes the zero section to $\hat{N}$. Moreover, $\hat{U}$ can be chosen to be an open set in $C_{1}^{1}$ and $\delta$ is compatible with the identifications $(0, \epsilon) \times \Sigma_{i} \stackrel{\Psi_{i}}{\cong} U_{i} \backslash\left\{z_{i}\right\}$ and the isomorphism $\nu(\hat{N}) \stackrel{\jmath}{\cong} \Lambda_{+}^{2} T^{*} \hat{N}$ given by Proposition 2.7 . 
Proof. Use the notation from the start of this section and of Corollary 6.3. Define $\hat{T}_{i}=\chi_{i}\left(\hat{S}_{i}\right)$. Then $\hat{T}_{i}$ is an open set in $M$ such that $U_{i} \backslash\left\{z_{i}\right\} \subseteq \hat{T}_{i} \subseteq$ $V_{i}$, since $Q_{i} \subseteq \hat{S}_{i}$ by Corollary 6.3 and $\chi_{i}\left(Q_{i}\right)=U_{i} \backslash\left\{z_{i}\right\}$.

Consider the bundle $\left(\Lambda_{+}^{2}\right)_{\chi_{i}^{*}(g)} T^{*}\left((0, \epsilon) \times \Sigma_{i}\right)$, where the notation $\left(\Lambda_{+}^{2}\right)_{h}$ indicates that the Hodge star is calculated using the metric $h$, and we identify $(0, \epsilon) \times \Sigma_{i}$ with $P_{i} \subseteq \mathbb{R}^{7}$ using the inclusion map $\iota_{i}$. Then

$$
\begin{aligned}
\jmath_{i}: \nu\left(P_{i}\right) & \longrightarrow\left(\Lambda_{+}^{2}\right)_{\chi_{i}^{*}(g)} T^{*} P_{i} \\
\left.v\right|_{r_{i} \sigma_{i}} & \left.\longmapsto\left(\left.\left.v\right|_{r_{i} \sigma_{i}} \cdot \chi_{i}^{*}(\varphi)\right|_{\Phi_{i}\left(r_{i}, \sigma_{i}\right)}\right)\right|_{T_{r_{i} \sigma_{i}} P_{i}}
\end{aligned}
$$

is an isomorphism because $U_{i} \backslash\left\{z_{i}\right\}$ is coassociative and thus $P_{i}$ is, with respect to $\left(\chi_{i}^{*}(\varphi), \chi_{i}^{*}(g)\right)$, and hence we may apply Proposition 2.7. Note also that

$$
\Psi_{i}^{*}:\left(\Lambda_{+}^{2}\right)_{g} T^{*}\left(U_{i} \backslash\left\{z_{i}\right\}\right) \longrightarrow\left(\Lambda_{+}^{2}\right)_{\chi_{i}^{*}(g)} T^{*}\left((0, \epsilon) \times \Sigma_{i}\right)
$$

is clearly a diffeomorphism. Therefore, let $\hat{U}_{i} \subseteq\left(\Lambda_{+}^{2}\right)_{g} T^{*}\left(U_{i} \backslash\left\{z_{i}\right\}\right)$ be such that $\Psi_{i}^{*}\left(\hat{U}_{i}\right)=\jmath_{i}\left(\hat{V}_{i}\right)$. Note that $\hat{U}_{i}$ is an open set in $C_{1}^{1}$ since $\hat{V}_{i}$ is by Corollary 6.3.

Define a diffeomorphism $\delta_{i}: \hat{U}_{i} \rightarrow \hat{T}_{i}$ such that the following commutes:

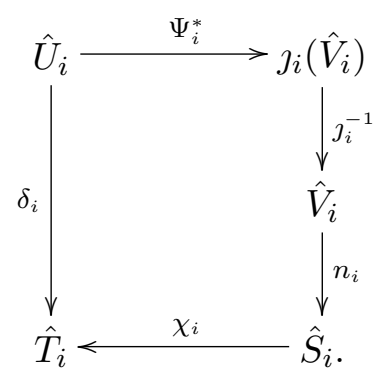

Interpolating smoothly over $K$, we extend $\bigcup_{i=1}^{s} \hat{U}_{i}$ and $\bigcup_{i=1}^{s} \hat{T}_{i}$ to $\hat{U}$ and $\hat{T}$ as required and extend the diffeomorphisms $\delta_{i}$ smoothly to a diffeomorphism $\delta: \hat{U} \rightarrow \hat{T}$ such that $\delta$ acts as the identity on $\hat{N}$, which is identified with the zero section in $\Lambda_{+}^{2} T^{*} \hat{N}$.

Note that we have a splitting $\left.T \hat{U}\right|_{(x, 0)}=T_{x} \hat{N} \oplus \Lambda_{+}^{2} T_{x}^{*} \hat{N}$ for all $x \in \hat{N}$. Thus we can consider $d \delta$ at $\hat{N}$ as a map from $T \hat{N} \oplus \Lambda_{+}^{2} T^{*} \hat{N}$ to $T \hat{N} \oplus \nu(\hat{N}) \cong$ $\left.T M\right|_{\hat{N}}$. Hence, we require in our extension of $\delta$ from $\delta_{i}$ to ensure that, in matrix notation,

$$
\left.d \delta\right|_{\hat{N}}=\left(\begin{array}{cc}
I & A \\
0 & J^{-1}
\end{array}\right),
$$


where $I$ is the identity and $A$ is arbitrary. This can be achieved because of the definition of $\delta_{i}$.

The compatibility of $\delta$ with $\jmath$ and $\Psi_{i}$ mentioned in the statement of the proposition, is given by (6.2) and the behaviour of $\left.d \delta\right|_{\hat{N}}$ stipulated in $(6.3)$.

Note. The open set $\hat{U}$ will be used frequently throughout the rest of the article.

We now define our deformation map for Problem 1. Let

$$
C_{\mathrm{loc}}^{k}(\hat{U})=\left\{\alpha \in C_{\mathrm{loc}}^{k}\left(\Lambda_{+}^{2} T^{*} \hat{N}\right): \Gamma_{\alpha} \subseteq \hat{U}\right\}
$$

where $\hat{U}$ is given in Proposition 6.4 and $\Gamma_{\alpha}$ is the graph of $\alpha$. We also adopt similar notation to define subsets of the spaces of forms described in $\S 4$, though we must be careful that the forms are continuous so that their graphs are well-defined. Moreover, we notice that the subset will be open whenever the Banach space embeds continuously in $C_{1}^{1}$.

Definition 6.5. Use the notation of Proposition 6.4. Let $\Gamma_{\alpha}$ be the graph of $\alpha \in C_{\text {loc }}^{1}(\hat{U})$ and let $\pi_{\alpha}: \hat{N} \rightarrow \Gamma_{\alpha}$ be given by $\pi_{\alpha}(x)=(x, \alpha(x))$. Let $f_{\alpha}=\delta \circ \pi_{\alpha}$ and let $\hat{N}_{\alpha}=f_{\alpha}(\hat{N}) \subseteq \hat{T}$. Define a map $F_{1}$ from $C_{\text {loc }}^{1}(\hat{U})$ to $C_{\text {loc }}^{0}\left(\Lambda^{3} T^{*} \hat{N}\right)$ by:

$$
F_{1}(\alpha)=f_{\alpha}^{*}\left(\left.\varphi\right|_{\hat{N}_{\alpha}}\right) .
$$

By Proposition 2.6, Ker $F_{1}$ is the set of $\alpha \in C_{\text {loc }}^{1}(\hat{U})$ such that $\hat{N}_{\alpha}$ is coassociative. Using [17, p. 731], which we are allowed to do by our choice of $\delta$, the linearization of $F_{1}$ at 0 acts on $\alpha \in C_{\mathrm{loc}}^{1}\left(\Lambda_{+}^{2} T^{*} \hat{N}\right)$ as

$$
\left.d F_{1}\right|_{0}(\alpha)=L_{1}(\alpha)=d \alpha
$$

Remark. The operator $L_{1}$ is not elliptic.

We want to study CS coassociative deformations $N_{\alpha}=\hat{N}_{\alpha} \sqcup\left\{z_{1}, \ldots, z_{s}\right\}$ with singularities at the same points as $N$ with the same tangent cones, so we restrict our choices of forms $\alpha \in C_{\text {loc }}^{1}(\hat{U})$. Since $\hat{N}_{\alpha}$ is supposed to be smooth and nonsingular, $\alpha \in C^{\infty}(\hat{U})$.

As $N_{\alpha}$ is a CS coassociative 4 -fold, there exist smooth maps $\left(\Phi_{\alpha}\right)_{i}$ : $(0, \epsilon) \times \Sigma_{i} \rightarrow B(0 ; \eta)$ satisfying (3.2) such that $\left(\Psi_{\alpha}\right)_{i}=\chi_{i} \circ\left(\Phi_{\alpha}\right)_{i}$ is a diffeomorphism onto an open subset of $\hat{N}_{\alpha}$ for all $i$ as in Definition 3.4. We are free to use $\chi_{i}$ because the tangent cones at the singularities of $N_{\alpha}$ must be 
the same as for $N$, so any $\mathrm{G}_{2}$ coordinate system near the singularities used to define $N_{\alpha}$ must be equivalent to the one given by $\chi_{i}$ for $i=1, \ldots, s$. Choose $\left(\Phi_{\alpha}\right)_{i}$ uniquely so as to satisfy a similar orthogonality condition to (6.1).

Use the notation of Corollary 6.3 and the proof of Proposition 6.4. Recall that we identify $(0, \epsilon) \times \Sigma_{i}$ with $P_{i}$ using $\iota_{i}$. By $(6.1), \Phi_{i}-\iota_{i}$ can be identified using $J_{i}$ with the graph of $\beta_{i} \in C^{\infty}\left(\left(\Lambda_{+}^{2}\right)_{\chi_{i}^{*}(g)} T^{*}\left((0, \epsilon) \times \Sigma_{i}\right)\right)$. Thus, if $\nabla_{i}$ is the Levi-Civita connection of the cone metric on $C_{i}$,

$$
\left|\nabla_{i}^{j} \beta_{i}\right|=O\left(r_{i}^{\lambda-j}\right) \quad \text { for } j \in \mathbb{N} \quad \text { as } r_{i} \longrightarrow 0
$$

by $(3.2)$, and therefore $\beta_{i} \in C_{\lambda}^{\infty}\left(\left(\Lambda_{+}^{2}\right)_{\chi_{i}^{*}(g)} T^{*}\left((0, \epsilon) \times \Sigma_{i}\right)\right)$.

We may similarly deduce, by the definition of $\delta, \Phi_{i}$ and $\left(\Phi_{\alpha}\right)_{i}$, that $\left(\Phi_{\alpha}\right)_{i}-\iota_{i}=\left(\left(\Phi_{\alpha}\right)_{i}-\Phi_{i}\right)+\left(\Phi_{i}-\iota_{i}\right)$ corresponds to the graph of $\Psi_{i}^{*}(\alpha)+\beta_{i}$ on $(0, \epsilon) \times \Sigma_{i}$, recalling that

$$
\Psi_{i}^{*}: \Lambda_{+}^{2} T^{*}\left(U_{i} \backslash\left\{z_{i}\right\}\right) \rightarrow\left(\Lambda_{+}^{2}\right)_{\chi_{i}^{*}(g)} T^{*}\left((0, \epsilon) \times \Sigma_{i}\right)
$$

is a diffeomorphism for all $i$. Since $N_{\alpha}$ has the same types of singularities as $N$, both $\beta_{i}$ and $\Psi_{i}^{*}(\alpha)+\beta_{i}$ lie in $C_{\lambda}^{\infty}\left(\left(\Lambda_{+}^{2}\right)_{\chi_{i}^{*}(g)} T^{*}\left((0, \epsilon) \times \Sigma_{i}\right)\right)$ for each $i$. Thus $\alpha$ must lie in $C_{\lambda}^{\infty}\left(\Lambda_{+}^{2} T^{*} \hat{N}\right)$.

We conclude that $\hat{N}_{\alpha}$ is a deformation of $\hat{N}$ in $\hat{T}$ with the same conical singularities if and only if $\alpha \in C_{\lambda}^{\infty}(\hat{U})$. We state this as a proposition.

Proposition 6.6. In the notation of Definitions 6.1 and $6.5, \mathcal{M}_{1}(N, \lambda)$ is locally homeomorphic to Ker $F_{1}=\left\{\alpha \in C_{\lambda}^{\infty}(\hat{U}): F_{1}(\alpha)=0\right\}$.

We define an associated map $G_{1}$ to $F_{1}$ which is a nonlinear elliptic operator at zero. This will be useful to prove regularity results.

Definition 6.7. Define $G_{1}: C_{\text {loc }}^{1}(\hat{U}) \times C_{\text {loc }}^{1}\left(\Lambda^{4} T^{*} \hat{N}\right) \rightarrow C_{\mathrm{loc}}^{0}\left(\Lambda^{3} T^{*} \hat{N}\right)$ by:

$$
G_{1}(\alpha, \beta)=F_{1}(\alpha)+d^{*} \beta
$$

This is a nonlinear first-order elliptic operator at $(0,0)$ since its linearization there acts on $(\alpha, \beta) \in C_{\text {loc }}^{1}\left(\Lambda_{+}^{2} T^{*} \hat{N} \oplus \Lambda^{4} T^{*} \hat{N}\right)$ as

$$
\left.d G_{1}\right|_{(0,0)}(\alpha, \beta)=d \alpha+d^{*} \beta .
$$

Note. If $G_{1}(\alpha, \beta)=0$ and $\beta \in C_{\lambda}^{\infty}\left(\Lambda^{4} T^{*} \hat{N}\right), * \beta$ is a harmonic function which decays with order $O\left(\rho^{\lambda}\right)$ as $\rho \rightarrow 0$. Since $\lambda>1$, * $\rightarrow \rightarrow 0$ as $\rho \rightarrow 0$ and hence, by the maximum principle for harmonic functions, it must be 0 . 
From the note, we deduce the following.

Proposition 6.8. $\operatorname{Ker} F_{1} \cong\left\{(\alpha, \beta) \in C_{\lambda}^{\infty}(\hat{U}) \times C_{\lambda}^{\infty}\left(\Lambda^{4} T^{*} \hat{N}\right): G_{1}(\alpha, \beta)=0\right\}$.

We conclude this section by stating and proving two results on regularity which are analogous to [16, Proposition 4.3] and [16, Proposition 4.14], respectively. The proof of the first is long and technical, but works by relating $F_{1}$ to functions on the cones $C_{i}$, then using scale equivariance properties of these new functions to prove regularity results using their restrictions to the links $\Sigma_{i}$.

Proposition 6.9. The map $F_{1}$ given in Definition 6.5 can be written as

$$
F_{1}(\alpha)(x)=d \alpha(x)+P_{F_{1}}(x, \alpha(x), \nabla \alpha(x))
$$

for $x \in \hat{N}$, where $P_{F_{1}}:\left\{(x, y, z):(x, y) \in \hat{U}, z \in T_{x}^{*} \hat{N} \otimes \Lambda_{+}^{2} T_{x}^{*} \hat{N}\right\} \rightarrow \Lambda^{3} T^{*} \hat{N}$ is a smooth map such that $P_{F_{1}}(x, y, z) \in \Lambda^{3} T_{x}^{*} \hat{N}$. Denote $P_{F_{1}}(x, \alpha(x)$, $\nabla \alpha(x))$ by $P_{F_{1}}(\alpha)(x)$ for convenience.

Let $\alpha \in C_{\text {loc }}^{1}(\hat{U})$ with $\|\alpha\|_{C_{1}^{1}}$ sufficiently small. If $\alpha \in C_{\lambda}^{\infty}(\hat{U}), P_{F_{1}}(\alpha) \in$ $C_{2 \lambda-2}^{\infty}\left(\Lambda^{3} T^{*} \hat{N}\right)$. Moreover, for each $k \in \mathbb{N}$, if $\alpha \in C_{\lambda}^{k+1}(\hat{U})$, then $P_{F_{1}}(\alpha) \in$ $C_{2 \lambda-2}^{k}\left(\Lambda^{3} T^{*} \hat{N}\right)$ and there exists a constant $c_{k}>0$ such that

$$
\left\|P_{F_{1}}(\alpha)\right\|_{C_{2 \lambda-2}^{k}} \leq c_{k}\|\alpha\|_{C_{\lambda}^{k+1}}^{2}
$$

Note. For each $k \in \mathbb{N}, C_{2 \lambda-2}^{k} \hookrightarrow C_{\lambda-1}^{k}$ as $\lambda>1$.

Proof. First, by the definition of $F_{1}, F_{1}(\alpha)(x)$ relates to the tangent space to $\Gamma_{\alpha}$ at $\pi_{\alpha}(x)$. Note that $T_{\pi_{\alpha}(x)} \Gamma_{\alpha}$ depends on both $\alpha(x)$ and $\nabla \alpha(x)$ and hence so must $F_{1}(\alpha)(x)$. We may then define $P_{F_{1}}$ by $(6.5)$ such that it is a smooth function of its arguments as claimed.

Recall the notation from the start of this section and that $(0, \epsilon) \times \Sigma_{i} \stackrel{\iota_{i}}{\cong} P_{i}$ $\subseteq \mathbb{R}^{7}$, where $\iota_{i}$ is the inclusion map. We argued before Proposition 6.6 that we may identify $\Phi_{i}-\iota_{i}$ on $(0, \epsilon) \times \Sigma_{i}$ with

$$
\beta_{i} \in C_{\lambda}^{\infty}\left(\left(\Lambda_{+}^{2}\right)_{\chi_{i}^{*}(g)} T^{*}\left((0, \epsilon) \times \Sigma_{i}\right)\right)
$$

for $i=1, \ldots, s$. Recall that

$$
\Psi_{i}^{*}: \Lambda_{+}^{2} T^{*}\left(U_{i} \backslash\left\{z_{i}\right\}\right) \longrightarrow\left(\Lambda_{+}^{2}\right)_{\chi_{i}^{*}(g)} T^{*}\left((0, \epsilon) \times \Sigma_{i}\right)
$$

is a diffeomorphism. Let $\alpha \in C_{\lambda}^{1}(\hat{U}), \alpha_{i}=\left.\alpha\right|_{U_{i} \backslash\left\{z_{i}\right\}}$ and $\gamma_{i}=\Psi_{i}^{*}\left(\alpha_{i}\right)$. 
For each $i$, define a function $F_{C_{i}}\left(\gamma_{i}+\beta_{i}\right)$ on $(0, \epsilon) \times \Sigma_{i}$ by

$$
F_{C_{i}}\left(\gamma_{i}+\beta_{i}\right)\left(r_{i}, \sigma_{i}\right)=F_{1}\left(\alpha_{i}\right)\left(\Psi_{i}\left(r_{i}, \sigma_{i}\right)\right)
$$

Further, define a smooth function $P_{C_{i}}$ by an equation analogous to (6.5):

$$
F_{C_{i}}\left(\gamma_{i}+\beta_{i}\right)\left(r_{i}, \sigma_{i}\right)=d\left(\gamma_{i}+\beta_{i}\right)\left(r_{i}, \sigma_{i}\right)
$$

$$
+P_{C_{i}}\left(\left(r_{i}, \sigma_{i}\right),\left(\gamma_{i}+\beta_{i}\right)\left(r_{i}, \sigma_{i}\right), \nabla\left(\gamma_{i}+\beta_{i}\right)\left(r_{i}, \sigma_{i}\right)\right) \text {. }
$$

We notice that $F_{C_{i}}$ and $P_{C_{i}}$ are only dependent on the cone $C_{i}$ and, rather trivially, on $\epsilon$. Therefore, because of this fact and our choice of $\delta$ in Proposition 6.4, these functions have scale equivariance properties. We may therefore derive equations and inequalities on $\{\epsilon\} \times \Sigma_{i}$ and deduce the result on all of $(0, \epsilon) \times \Sigma_{i}$ by introducing appropriate scaling factors of $r_{i}$.

Now, since $\alpha=0$ corresponds to our coassociative 4 -fold $\hat{N}, F_{1}(0)=0$. Adopting similar notation for $P_{C_{i}}\left(\beta_{i}\right)$ as for $P_{F_{1}}\left(\alpha_{i}\right)$, we see that

$$
F_{C_{i}}\left(\beta_{i}\right)=d \beta_{i}+P_{C_{i}}\left(\beta_{i}\right)=0 \text {, }
$$

by (6.7). Using (6.5) to (6.9), we deduce that

$$
\begin{aligned}
P_{F_{1}}\left(\alpha_{i}\right) & =d \beta_{i}+P_{C_{i}}\left(\gamma_{i}+\beta_{i}\right)=d \beta_{i}+P_{C_{i}}\left(\gamma_{i}+\beta_{i}\right)-\left(d \beta_{i}+P_{C_{i}}\left(\beta_{i}\right)\right) \\
& =P_{C_{i}}\left(\gamma_{i}+\beta_{i}\right)-P_{C_{i}}\left(\beta_{i}\right)
\end{aligned}
$$

We then calculate

$$
\begin{aligned}
P_{C_{i}}\left(\gamma_{i}+\beta_{i}\right)-P_{C_{i}}\left(\beta_{i}\right) & =\int_{0}^{1} \frac{d}{d t} P_{C_{i}}\left(t \gamma_{i}+\beta_{i}\right) d t \\
& =\int_{0}^{1} \gamma_{i} \cdot \frac{\partial P_{C_{i}}}{\partial y}\left(t \gamma_{i}+\beta_{i}\right)+\nabla_{i} \gamma_{i} \cdot \frac{\partial P_{C_{i}}}{\partial z}\left(t \gamma_{i}+\beta_{i}\right) d t,
\end{aligned}
$$

recalling that $P_{C_{i}}$ is a function of three variables $x, y$ and $z$ and that $\nabla_{i}$ is the Levi-Civita connection of the cone metric $g_{i}$ on $C_{i}$. Using Taylor's Theorem,

$$
\begin{aligned}
& P_{C_{i}}\left(\gamma_{i}+\beta_{i}\right) \\
& \quad=P_{C_{i}}\left(\beta_{i}\right)+\gamma_{i} \cdot \frac{\partial P_{C_{i}}}{\partial y}\left(\beta_{i}\right)+\nabla_{i} \gamma_{i} \cdot \frac{\partial P_{C_{i}}}{\partial z}\left(\beta_{i}\right)+O\left(r_{i}^{-2}\left|\gamma_{i}\right|^{2}+\left|\nabla_{i} \gamma_{i}\right|^{2}\right)
\end{aligned}
$$

when $\left|\gamma_{i}\right|$ and $\left|\nabla_{i} \gamma_{i}\right|$ are small.

Since $\left.d F_{1}\right|_{0}\left(\alpha_{i}\right)=d \alpha_{i},\left.d F_{C_{i}}\right|_{\beta_{i}}\left(\gamma_{i}+\beta_{i}\right)=d \gamma_{i}$ and hence $\left.d P_{C_{i}}\right|_{\beta_{i}}=0$. Thus, the first derivatives of $P_{C_{i}}$ with respect to $y$ and $z$ must vanish at 
$\beta_{i}$ by (6.12). Therefore, given small $\nu>0$, there exists a constant $A_{0}>0$ such that

$$
\begin{aligned}
& \left|\frac{\partial P_{C_{i}}}{\partial y}\left(t \gamma_{i}+\beta_{i}\right)\right| \leq A_{0}\left(r_{i}^{-2}\left|\gamma_{i}\right|+r_{i}^{-1}\left|\nabla_{i} \gamma_{i}\right|\right), \text { and } \\
& \left|\frac{\partial P_{C_{i}}}{\partial z}\left(t \gamma_{i}+\beta_{i}\right)\right| \leq A_{0}\left(r_{i}^{-1}\left|\gamma_{i}\right|+\left|\nabla_{i} \gamma_{i}\right|\right)
\end{aligned}
$$

for $t \in[0,1]$ whenever

$$
r_{i}^{-1}\left|\gamma_{i}\right|, \quad r_{i}^{-1}\left|\beta_{i}\right|, \quad\left|\nabla_{i} \gamma_{i}\right| \quad \text { and } \quad\left|\nabla_{i} \beta_{i}\right| \leq \nu
$$

We determine the appropriate factors of $r_{i}$ by considering the scaling properties of $P_{C_{i}}$ and $\gamma_{i}$ under changes in $r_{i}$.

By (6.4), $r_{i}^{-1}\left|\beta_{i}\right|$ and $\left|\nabla_{i} \beta_{i}\right|$ tend to zero as $r_{i} \rightarrow 0$. We can thus ensure that (6.14) is satisfied by the $\beta_{i}$ components by making $\epsilon$ smaller. Hence, (6.14) holds if $\left\|\gamma_{i}\right\|_{C_{1}^{1}} \leq \nu$. Therefore, putting estimates (6.13) in (6.11) and using (6.10),

$$
\left|P_{F_{1}}\left(\alpha_{i}\right)\right|=\left|P_{C_{i}}\left(\gamma_{i}+\beta_{i}\right)-P_{C_{i}}\left(\beta_{i}\right)\right| \leq A_{0}\left(r_{i}^{-1}\left|\gamma_{i}\right|+\left|\nabla_{i} \gamma_{i}\right|\right)^{2}
$$

whenever $\left\|\gamma_{i}\right\|_{C_{1}^{1}} \leq \nu$. As $r_{i} \rightarrow 0$ the terms in the bracket on the righthand side of $(6.15)$ are of order $O\left(r_{i}^{\lambda-1}\right)$ by (6.4). Thus, $\left|P_{F_{1}}\left(\alpha_{i}\right)\right|$ is of order $O\left(r_{i}^{2 \lambda-2}\right)$ as $r_{i} \rightarrow 0$ for $i=1, \ldots, s$. We deduce that $\left|P_{F_{1}}(\alpha)\right|$ is of order $O\left(\rho^{2 \lambda-2}\right)$ as $\rho \rightarrow 0$ if $\|\alpha\|_{C_{1}^{1}}$ sufficiently small. Moreover, there exists a constant $c_{0}$ such that

$$
\sup _{\hat{N}}\left|\rho^{2-2 \lambda} P_{F_{1}}(\alpha)\right| \leq c_{0}\left(\sum_{j=0}^{1} \sup _{\hat{N}}\left|\rho^{j-\lambda} \nabla^{j} \alpha\right|\right)^{2}
$$

that is, (6.6) holds for $k=0$.

We now consider the first derivative of $P_{F_{1}}$ and, in doing so, provide the method to tackle higher derivatives as well. Suppose $\alpha \in C_{\lambda}^{2}(\hat{U})$ and $\|\alpha\|_{C_{1}^{1}}$ is sufficiently small that $\left\|\gamma_{i}\right\|_{C_{1}^{1}} \leq \nu$ for all $i$. From (6.11) we calculate

$$
\begin{aligned}
& \nabla_{i}\left(P_{C_{i}}\left(\gamma_{i}+\beta_{i}\right)-P_{C_{i}}\left(\beta_{i}\right)\right) \\
& \quad=\int_{0}^{1} \nabla_{i}\left(\gamma_{i} \cdot \frac{\partial P_{C_{i}}}{\partial y}\left(t \gamma_{i}+\beta_{i}\right)+\nabla_{i} \gamma_{i} \cdot \frac{\partial P_{C_{i}}}{\partial z}\left(t \gamma_{i}+\beta_{i}\right)\right) d t
\end{aligned}
$$




$$
\begin{aligned}
= & \int_{0}^{1} \nabla_{i} \gamma_{i} \cdot \frac{\partial P_{C_{i}}}{\partial y}+\gamma_{i} \cdot\left(\nabla_{i}\left(t \gamma_{i}+\beta_{i}\right) \cdot \frac{\partial^{2} P_{C_{i}}}{\partial y^{2}}+\nabla_{i}^{2}\left(t \gamma_{i}+\beta_{i}\right) \cdot \frac{\partial^{2} P_{C_{i}}}{\partial y \partial z}\right) \\
& +\nabla_{i}^{2} \gamma_{i} \cdot \frac{\partial P_{C_{i}}}{\partial z}+\nabla_{i} \gamma_{i} \cdot\left(\nabla_{i}\left(t \gamma_{i}+\beta_{i}\right) \cdot \frac{\partial^{2} P_{C_{i}}}{\partial z \partial y}+\nabla_{i}^{2}\left(t \gamma_{i}+\beta_{i}\right) \cdot \frac{\partial^{2} P_{C_{i}}}{\partial z^{2}}\right) d t .
\end{aligned}
$$

There exists a constant $A_{1}>0$ such that (6.13) holds with $A_{0}$ replaced by $A_{1}$ and, for $t \in[0,1]$,

$$
\left|\frac{\partial^{2} P_{C_{i}}}{\partial y^{2}}\left(t \gamma_{i}+\beta_{i}\right)\right|, \quad\left|\frac{\partial^{2} P_{C_{i}}}{\partial y \partial z}\left(t \gamma_{i}+\beta_{i}\right)\right| \text { and } \quad\left|\frac{\partial^{2} P_{C_{i}}}{\partial z^{2}}\left(t \gamma_{i}+\beta_{i}\right)\right| \leq A_{1},
$$

since the second derivatives of $P_{C_{i}}$ are continuous functions defined on the closed bounded set given by $\left\|\gamma_{i}\right\|_{C_{1}^{1}} \leq \nu$. We deduce that

$$
\left|\nabla\left(P_{F_{1}}\left(\alpha_{i}\right)\right)\right|=\left|\nabla_{i}\left(P_{C_{i}}\left(\gamma_{i}+\beta_{i}\right)-P_{C_{i}}\left(\beta_{i}\right)\right)\right| \leq A_{1} r_{i}\left(\sum_{j=0}^{2} r_{i}^{j-2}\left|\nabla_{i}^{j} \gamma_{i}\right|\right)^{2} .
$$

Therefore, $\left|\nabla\left(P_{F_{1}}\left(\alpha_{i}\right)\right)\right|$ is of order $O\left(r_{i}^{2 \lambda-3}\right)$ as $r_{i} \rightarrow 0$, and (6.6) holds for $k=1$.

In general, if $\alpha \in C_{\lambda}^{k+1}(\hat{U})$ with $\|\alpha\|_{C_{1}^{1}}$ sufficiently small, we have

$$
\left|\nabla^{k}\left(P_{F_{1}}\left(\alpha_{i}\right)\right)\right| \leq A_{k} r_{i}^{k}\left(\sum_{j=0}^{k+1} r_{i}^{j-(k+1)}\left|\nabla_{i}^{j} \gamma_{i}\right|\right)^{2}
$$

for some $A_{k}>0$. The result follows.

We now consider the regularity of solutions to the nonlinear elliptic equation $G_{1}(\alpha, \beta)=0$ near $(0,0)$. Our result is proved using a standard "bootstrap" argument.

Proposition 6.10. Let $(\alpha, \beta) \in L_{k+1, \lambda}^{p}(\hat{U}) \times L_{k+1, \lambda}^{p}\left(\Lambda^{4} T^{*} \hat{N}\right)$ for some $p>$ 4 and $k \geq 2$. If $G_{1}(\alpha, \beta)=0$ and $\|\alpha\|_{C_{1}^{1}}$ is sufficiently small, where $G_{1}$ is given by Definition 6.7 , then $(\alpha, \beta) \in C_{\lambda}^{\infty}(\hat{U}) \times C_{\lambda}^{\infty}\left(\Lambda^{4} T^{*} \hat{N}\right)$.

\section{Notes.}

(a) The conditions $p>4$ and $k \geq 2$ ensure, by Theorem 4.4 , that $L_{k+1, \lambda}^{p} \hookrightarrow$ $C_{\lambda}^{2} \hookrightarrow C_{1}^{1}$. We need to control at least the first two derivatives of forms in the kernel of $G_{1}$ since, although $G_{1}$ is a first-order operator, the proof uses a related second-order elliptic operator. 
(b) Since $\hat{U}$ is an open subset of $C_{1}^{1}$ and we are free to make $\hat{U}$ smaller if necessary, we can always ensure that $\alpha \in L_{k+1, \lambda}^{p}(\hat{U})$ has small enough $C_{1}^{1}$ norm for Proposition 6.10 to apply.

Proof. Since $F_{1}$ smoothly depends on $\alpha$ and $\nabla \alpha, G_{1}$ is a smooth function of $\alpha, \beta, \nabla \alpha$ and $\nabla \beta$. We apply [20, Theorem 6.8.1], which is a general regularity result for $C_{\text {loc }}^{1}$ solutions of nonlinear elliptic equations, to conclude that $\alpha$ and $\beta$ are smooth. However, we want more than this: the derivatives of $\alpha$ and $\beta$ must decay at the required rates.

Recall the note after Definition 6.7, which shows that $G_{1}(\alpha, \beta)=0$ implies that $\beta=0$. Thus $\beta \in C_{\lambda}^{\infty}\left(\Lambda^{4} T^{*} \hat{N}\right)$ trivially.

For the following argument, we find it useful to work with weighted Hölder spaces. By Theorem 4.4, $\alpha \in C_{\lambda}^{k, a}(\hat{U})$ with $a=1-4 / p \in(0,1)$ since $p>4$. We also know that $d^{*}\left(G_{1}(\alpha, \beta)\right)=d^{*}\left(F_{1}(\alpha)\right)=0$. Hence, if $\pi_{\Lambda_{+}^{2}}$ is the projection from 2-forms to their self-dual part,

$$
\tilde{F}_{1}(\alpha)=\pi_{\Lambda_{+}^{2}}\left(d^{*}\left(F_{1}(\alpha)\right)\right)=0
$$

is a nonlinear elliptic equation at 0 , since $\left.d F_{1}\right|_{0}(\alpha)=d \alpha$.

As $\tilde{F}_{1}(\alpha)$ is linear in the second derivative of $\alpha$, we see that

$$
\tilde{F}_{1}(\alpha)(x)=R(x, \alpha(x), \nabla \alpha(x)) \nabla^{2} \alpha(x)+E(x, \alpha(x), \nabla \alpha(x)),
$$

where $R(x, \alpha(x), \nabla \alpha(x))$ and $E(x, \alpha(x), \nabla \alpha(x))$ are smooth functions of their arguments. Define

$$
S_{\alpha}(\gamma)(x)=R(x, \alpha(x), \nabla \alpha(x)) \nabla^{2} \gamma(x)
$$

for $\gamma \in C_{\text {loc }}^{2}\left(\Lambda_{+}^{2} T^{*} \hat{N}\right)$. Then $S_{\alpha}$ is a smooth, linear, elliptic, second-order operator, whose coefficients depend on $x, \alpha(x)$ and $\nabla \alpha(x)$. These coefficients therefore lie in $C_{\mathrm{loc}}^{k-1, a}$. We also notice that

$$
S_{\alpha}(\alpha)(x)=-E(x, \alpha(x), \nabla \alpha(x)) \in C_{2 \lambda-3}^{k-2, a}\left(\Lambda_{+}^{2} T^{*} \hat{N}\right) \subseteq C_{\lambda-2}^{k-2, a}\left(\Lambda_{+}^{2} T^{*} \hat{N}\right),
$$

using the fact that $d^{*}\left(P_{F}(\alpha)\right) \in C_{2 \lambda-3}^{k-2, a}$ by Proposition 6.9 and $\lambda>1$. However, $E(x, \alpha(x), \nabla \alpha(x))$ only depends on $\alpha$ and $\nabla \alpha$ and is at worst quadratic in these quantities by Proposition 6.9, so it must in fact lie in $C_{\lambda-2}^{k-1, a}$ since we are given control on the decay of the first $k$ derivatives of $\alpha$ near the singularities of $N$.

In [19], a regularity result is given for linear elliptic operators acting between weighted Hölder spaces, whose coefficients locally lie in a suitable 
Hölder space. This result can also be found in [16, Theorem 4.12]. We deduce that, if $\gamma \in C_{\lambda}^{2}$ and $S_{\alpha}(\gamma) \in C_{\lambda-2}^{k-1, a}$, then $\gamma \in C_{\lambda}^{k+1, a}$. Since $k \geq 2, \alpha$ and $S_{\alpha}(\alpha)$ satisfy these conditions by the discussion above. Thus $\alpha \in C_{\lambda}^{k+1, a}$ only knowing a priori that $\alpha \in C_{\lambda}^{k, a}$. We proceed by induction to show that $\alpha \in C_{\lambda}^{l, a}$ for all $l \geq k$.

\subsection{Problem 2: moving singularities and fixed $G_{2}$ structure}

For this problem, we again consider deformations of $N$ in $(M, \varphi, g)$ which are CS coassociative 4 -folds at $s$ points with the same rate and cones at the singularities, but now we allow the singular points and tangent cones at those points to differ from those of $N$. However, we still assume that the $\mathrm{G}_{2}$ structure on $M$ is fixed. We again define the moduli space formally and recall the notation introduced at the start of the section.

Definition 6.11. The moduli space of deformations $\mathcal{M}_{2}(N, \lambda)$ for Problem 2 is the set of $N^{\prime}$ in $(M, \varphi, g)$ which are CS coassociative 4-folds at $z_{1}^{\prime}, \ldots, z_{s}^{\prime}$ with rate $\lambda$, having cone $C_{i}$ and tangent cone $\hat{C}_{i}^{\prime}$ at $z_{i}^{\prime}$ for all $i$, such that there exists a diffeomorphism $h: M \rightarrow M$, isotopic to the identity, such that $h\left(z_{i}\right)=z_{i}^{\prime}$ for $i=1, \ldots, s,\left.h\right|_{N}: N \rightarrow N^{\prime}$ is a homeomorphism and $\left.h\right|_{\hat{N}}: \hat{N} \rightarrow N^{\prime} \backslash\left\{z_{1}^{\prime}, \ldots, z_{s}^{\prime}\right\}$ is a diffeomorphism.

Here it is more difficult to create a local description of the moduli space which is compatible with our analytic framework. What one would consider "intuitive" approaches do not, as far as the author is aware, bear fruit. We therefore follow what is, at first sight, a slightly indirect route.

Let $\left\{B_{i}: i=1, \ldots, s\right\}$ be a collection of pairwise disjoint open sets in $M$ such that $z_{i} \in B_{i}$. Let $B=\prod_{i=1}^{s} B_{i}$. For each $\mathbf{z}^{\prime}=\left(z_{1}^{\prime}, \ldots, z_{s}^{\prime}\right) \in B$, we have a family $I\left(\mathbf{z}^{\prime}\right)$ of choices of $s$-tuples $\zeta^{\prime}=\left(\zeta_{1}^{\prime}, \ldots, \zeta_{s}^{\prime}\right)$ of isomorphisms $\zeta_{i}^{\prime}: \mathbb{R}^{7} \rightarrow T_{z_{i}^{\prime}} M$ identifying $\left(\varphi_{0}, g_{0}\right)$ with $\left(\left.\varphi\right|_{T_{z_{i}^{\prime}} M},\left.g\right|_{T_{z_{i}^{\prime}} M}\right)$. Clearly, for each $\mathbf{z}^{\prime} \in B, I\left(\mathbf{z}^{\prime}\right) \cong \mathrm{G}_{2}^{s}$. We thus make the following definition.

Definition 6.12. The translation space is

$$
\mathcal{T}=\left\{\left(\mathbf{z}^{\prime}, \boldsymbol{\zeta}^{\prime}\right): \mathbf{z}^{\prime} \in B, \boldsymbol{\zeta}^{\prime} \in I\left(\mathbf{z}^{\prime}\right)\right\}
$$

It is a principal $\mathrm{G}_{2}^{s}$ bundle over $B$ and hence is a smooth manifold.

We now deal with the issue that the cone $\iota_{i}\left(C_{i}\right)$ in $\mathbb{R}^{7}$ may have $\mathrm{G}_{2}$ symmetries, which means that different choices of $\zeta^{\prime}$ could give the same set of corresponding tangent cones. Let $\mathrm{H}_{i}$ denote the Lie subgroup of $\mathrm{G}_{2}$ 
preserving $\iota_{i}\left(C_{i}\right)$ in $\mathbb{R}^{7}$ and let $\mathrm{H}=\prod_{i=1}^{s} \mathrm{H}_{i} \subseteq \mathrm{G}_{2}^{s}$. Then $\mathrm{H}$ acts freely on $\mathcal{T}$ by

$$
\left(\mathbf{z}^{\prime}, \zeta^{\prime}\right) \longmapsto\left(\mathbf{z}^{\prime},\left(\zeta_{1}^{\prime} \circ A_{1}^{-1}, \ldots, \zeta_{s}^{\prime} \circ A_{s}^{-1}\right)\right),
$$

where $\left(A_{1}, \ldots, A_{s}\right) \in \mathrm{H}$. Thus there exists an H-orbit through $(\mathbf{z}, \boldsymbol{\zeta})$ in $\mathcal{T}$, where

$$
\mathbf{z}=\left(z_{1}, \ldots, z_{s}\right) \text { and } \boldsymbol{\zeta}=\left(\zeta_{1}, \ldots, \zeta_{s}\right)
$$

Define $\hat{\mathcal{T}}$ to be a small open ball in $\mathbb{R}^{n}$ containing 0 , where $n=\operatorname{dim} \mathcal{T}-$ $\operatorname{dim} \mathrm{H}$, and let $h_{\hat{\mathcal{T}}}: \hat{\mathcal{T}} \rightarrow \mathcal{T}$ be an embedding with $h_{\hat{\mathcal{T}}}(0)=(\mathbf{z}, \boldsymbol{\zeta})$ such that $h_{\hat{\mathcal{T}}}(\hat{\mathcal{T}})$ is transverse to the H-orbit through $(\mathbf{z}, \boldsymbol{\zeta})$. Write $h_{\hat{\mathcal{T}}}(t)=(\mathbf{z}(t), \boldsymbol{\zeta}(t))$ for $t \in \hat{\mathcal{T}}$, with $\mathbf{z}(0)=\mathbf{z}$ and $\boldsymbol{\zeta}(0)=\boldsymbol{\zeta}$.

We shall use the notation from this definition throughout the rest of the article.

\section{Notes.}

(a) If $t, t^{\prime} \in \hat{\mathcal{T}}$, with $t \neq t^{\prime}$, are such that $\mathbf{z}(t)=\mathbf{z}\left(t^{\prime}\right)$, the $s$-tuples of tangent cones, $\left\{\hat{C}_{1}(t), \ldots, \hat{C}_{s}(t)\right\}$ and $\left\{\hat{C}_{1}\left(t^{\prime}\right), \ldots, \hat{C}_{s}\left(t^{\prime}\right)\right\}$, are distinct.

(b) $\hat{\mathcal{T}}$ is an open ball in $\mathbb{R}^{n} \cong T_{0} \hat{\mathcal{T}}$ and hence can be considered as an open subset of $T_{0} \hat{\mathcal{T}}$.

We use $\hat{\mathcal{T}}$ to extend $N$ to a family of nearby CS 4-folds and provide an analogue to Proposition 6.4 for Problem 2. In defining $N$, we chose a $\mathrm{G}_{2}$ coordinate system $\left\{\chi_{i}: B(0 ; \eta) \rightarrow V_{i}: i=1, \ldots, s\right\}$ with $\left.d \chi_{i}\right|_{0}=\zeta_{i}$ for $i=1, \ldots, s$. Extend this to a smooth family of $\mathrm{G}_{2}$ coordinate systems

$$
\left\{\left\{\chi_{i}(t): B(0 ; \eta) \rightarrow V_{i}(t): i=1, \ldots, s\right\}: t \in \hat{\mathcal{T}}\right\}
$$

where $V_{i}(t)$ is an open set in $M$ containing $z_{i}(t), \chi_{i}(t)(0)=z_{i}(t),\left.d \chi_{i}(t)\right|_{0}=$ $\zeta_{i}(t), \chi_{i}(0)=\chi_{i}$ and $V_{i}(0)=V_{i}$ for $i=1, \ldots, s$.

Proposition 6.13. Use the notation of Proposition 6.4 and Definition 6.12.

(a) There exists a family $\mathcal{N}=\{N(t): t \in \hat{\mathcal{T}}\}$ of $C S$ 4-folds in $M$, with $N(0)=N$, such that $N(t)$ has a singularity at $z_{i}(t)$, for $i=1, \ldots, s$, with rate $\lambda$, cone $C_{i}$ and tangent cone $\hat{C}_{i}(t)=\left(\zeta_{i}(t) \circ \iota_{i}\right)\left(C_{i}\right)$.

(b) Let $\hat{N}(t)=N(t) \backslash\left\{z_{1}(t), \ldots, z_{s}(t)\right\}$ and write $N(t)=K(t) \sqcup \bigsqcup_{i=1}^{s}$ $U_{i}(t)$, where $K(t)$ is compact and $U_{i}(t) \backslash\left\{z_{i}(t)\right\} \cong(0, \epsilon) \times \Sigma_{i}$ for all $i$, in the obvious way, ensuring that $K(0)=K$ and $U_{i}(0)=U_{i}$. 
For $t \in \hat{\mathcal{T}}$, there exist open sets $\hat{T}(t) \subseteq M$ containing $\hat{N}(t)$ and diffeomorphisms $\delta(t): \hat{U} \rightarrow \hat{T}(t)$ taking the zero section to $\hat{N}(t)$, varying smoothly in $t$, with $\hat{T}(0)=\hat{T}$ and $\delta(0)=\delta$. Moreover, $\delta(t)$ is compatible with the identifications $U_{i}(t) \backslash\left\{z_{i}(t)\right\} \cong(0, \epsilon) \times \Sigma_{i}$ for all $i$.

Remark. $\mathcal{N}$ does not necessarily consist of CS coassociative 4-folds and $\delta(t)$ is not required to be compatible with the isomorphism $\nu(\hat{N}) \cong \Lambda_{+}^{2} T^{*} \hat{N}$ for $t \neq 0$.

Proof. Use the notation from Corollary 6.3 and the proof of Proposition 6.4. For $t \in \hat{\mathcal{T}}$, define $\hat{T}_{i}(t)=\chi_{i}(t)\left(\hat{S}_{i}\right)$ and

$$
U_{i}(t)=\left(\chi_{i}(t) \circ \Phi_{i}\left((0, \epsilon) \times \Sigma_{i}\right)\right) \cup\left\{z_{i}(t)\right\}
$$

for $i=1, \ldots, s$. Then $\hat{T}_{i}(t)$ contains $U_{i}(t) \backslash\left\{z_{i}(t)\right\}$. Define a diffeomorphism $\delta_{i}(t)$ such that the following diagram commutes:

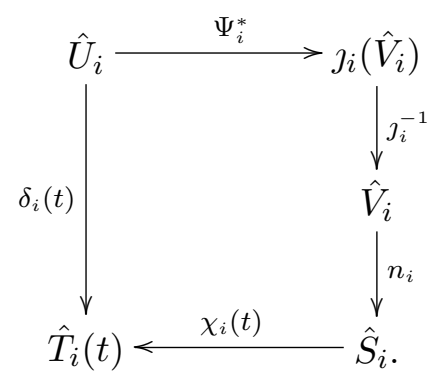

Interpolating smoothly over $K$, we extend $\bigcup_{i=1}^{s} \hat{T}_{i}(t)$ to $\hat{T}(t)$ and $\delta_{i}(t)$ to $\delta(t)$.

Let $e(t)=\left.\delta(t)\right|_{\hat{N}}$ and define $\hat{N}(t)=e(t)(\hat{N})$. Then $e(t): \hat{N} \rightarrow \hat{N}(t)$ is a diffeomorphism for all $t \in \hat{\mathcal{T}}$ and $e(0)$ is the identity. Let $N(t)=\hat{N}(t) \cup$ $\left\{z_{1}(t), \ldots, z_{s}(t)\right\}$. We then have a family $\mathcal{N}=\{N(t): t \in \hat{\mathcal{T}}\}$ as claimed. Note that $K(t)=e(t)(K)$.

By the construction of $\delta(t)$ and the family $\mathcal{N}$, it is clear that the proposition is proved, where the compatibility conditions on $\delta(t)$ are given by (6.16).

We can now define the deformation map for Problem 2.

Definition 6.14. Use the notation of Definition 6.5 and Proposition 6.13. For $t \in \hat{\mathcal{T}}$, let $f_{\alpha}(t)=\delta(t) \circ \pi_{\alpha}, \hat{N}_{\alpha}(t)=f_{\alpha}(t)(\hat{N})$ and $N_{\alpha}(t)=\hat{N}_{\alpha}(t) \cup$ 
$\left\{z_{1}(t), \ldots, z_{s}(t)\right\}$. Define $F_{2}$ from $C_{\text {loc }}^{1}(\hat{U}) \times \hat{\mathcal{T}}$ to $C_{\text {loc }}^{0}\left(\Lambda^{3} T^{*} \hat{N}\right)$ by:

$$
F_{2}(\alpha, t)=f_{\alpha}(t)^{*}\left(\left.\varphi\right|_{\hat{N}_{\alpha}(t)}\right)
$$

The linearization of $F_{2}$ at $(0,0)$ acts as

$$
\left.d F_{2}\right|_{(0,0)}:(\alpha, t) \longmapsto d \alpha+L_{2}(t)
$$

where $\alpha \in C_{\text {loc }}^{1}\left(\Lambda_{+}^{2} T^{*} \hat{N}\right), t \in T_{0} \hat{\mathcal{T}}$ and $L_{2}$ is a linear map into the space of smooth exact 3 -forms on $\hat{N}$ since $\varphi$ is exact near $\hat{N}$.

Remark. By construction $F_{2}(\alpha, 0)=F_{1}(\alpha)$, where $F_{1}$ is given in Definition 6.5.

Clearly, $\operatorname{Ker} F_{2}$ is the set of $\alpha \in C_{\text {loc }}^{1}(\hat{U})$ and $t \in \hat{\mathcal{T}}$ such that $\hat{N}_{\alpha}(t)$ is coassociative. However, we have not yet encoded the information that $N_{\alpha}(t)$ is CS with rate $\lambda$. This is the subject of the next proposition.

Proposition 6.15. In the notation of Definitions 6.11 and $6.14, \mathcal{M}_{2}(N, \lambda)$ is locally homeomorphic to $\operatorname{Ker} F_{2}=\left\{(\alpha, t) \in C_{\lambda}^{\infty}(\hat{U}) \times \hat{\mathcal{T}}: F_{2}(\alpha, t)=0\right\}$.

Proof. For each $t \in \hat{\mathcal{T}}$, we are in the situation of Problem 1 in the sense that we want coassociative deformations $\hat{N}_{\alpha}(t)$ of $\hat{N}(t)$, defined by a self-dual 2 -form $\alpha$, which have the same singular points, cones and tangent cones as $\hat{N}(t)$. It is thus clear that $\alpha \in C_{\lambda}^{\infty}(\hat{U})$ by Proposition 6.6.

We now introduce an associated map $G_{2}$ to $F_{2}$.

Definition 6.16. Use the notation of Definition 6.14. Define $G_{2}: C_{\text {loc }}^{1}(\hat{U}) \times$ $C_{\text {loc }}^{1}\left(\Lambda^{4} T^{*} \hat{N}\right) \times \hat{\mathcal{T}} \rightarrow C_{\text {loc }}^{0}\left(\Lambda^{3} T^{*} \hat{N}\right)$ by

$$
G_{2}(\alpha, \beta, t)=F_{2}(\alpha, t)+d^{*} \beta
$$

so that $\left.d G_{2}\right|_{(0,0,0)}:(\alpha, \beta, t) \longmapsto d \alpha+d^{*} \beta+L_{2}(t)$.

We then have an analogous result to Proposition 6.8, which follows in exactly the same fashion because $F_{2}(\alpha, t)$ is exact.

\section{Proposition 6.17.}

$\operatorname{Ker} F_{2} \cong\left\{(\alpha, \beta, t) \in C_{\lambda}^{\infty}(\hat{U}) \times C_{\lambda}^{\infty}\left(\Lambda^{4} T^{*} \hat{N}\right) \times \hat{\mathcal{T}}: G_{2}(\alpha, \beta, t)=0\right\}$. 
The next result studies the regularity of the kernel of $G_{2}$ near $(0,0,0)$ and is the analogue of Proposition 6.10.

Proposition 6.18. Let $(\alpha, \beta, t) \in L_{k+1, \lambda}^{p}(\hat{U}) \times L_{k+1, \lambda}^{p}\left(\Lambda^{4} T^{*} \hat{N}\right) \times \hat{\mathcal{T}}$, where $p>4$ and $k \geq 2$. If $G_{2}(\alpha, \beta, t)=0$, where $G_{2}$ is given in Definition 6.16, and $\|\alpha\|_{C_{1}^{1}}$ and $t$ are sufficiently small, then $(\alpha, \beta) \in C_{\lambda}^{\infty}(\hat{U}) \times C_{\lambda}^{\infty}$ $\left(\Lambda^{4} T^{*} \hat{N}\right)$.

Note. We can always ensure that $t$ is sufficiently small by shrinking $\hat{\mathcal{T}}$.

Proof. Recall the proof of Proposition 6.10 and Definition 6.16.

First, $d\left(G_{2}(\alpha, \beta, t)\right)=\Delta \beta=0$ since $F_{2}(\alpha, t)$ is exact. Thus, $\beta=0$ by the maximum principle, so it is trivially smooth.

Note that $d^{*}\left(G_{2}(\alpha, \beta, t)\right)=d^{*}\left(F_{2}(\alpha, t)\right)=0$. Hence,

$$
\tilde{F}_{2}(\alpha, t)=\pi_{\Lambda_{+}^{2}}\left(d^{*}\left(F_{2}(\alpha, t)\right)\right)=0
$$

is a nonlinear equation on $\alpha$ which is linear in $\nabla^{2} \alpha$. We can thus write

$$
\tilde{F}_{2}(\alpha, t)(x)=R_{t}(x, \alpha(x), \nabla \alpha(x)) \nabla^{2} \alpha(x)+E_{t}(x, \alpha(x), \nabla \alpha(x)),
$$

where $R_{t}$ and $E_{t}$ are smooth functions of their arguments. If we define

$$
S_{(\alpha, t)}(\gamma)(x)=R_{t}(x, \alpha(x), \nabla \alpha(x)) \nabla^{2} \gamma(x)
$$

then $S_{(\alpha, t)}$ is a linear differential operator acting on $\gamma \in C_{\text {loc }}^{2}\left(\Lambda_{+}^{2} T^{*} \hat{N}\right)$, with coefficients which lie in $C_{\mathrm{loc}}^{k-1, a}$. The ellipticity of $S_{\alpha}=S_{(\alpha, 0)}$ results from the coassociativity of $\hat{N}$. Ellipticity is an open condition so, although $\hat{N}(t)$ is not necessarily coassociative, the fact that it is "close" to being coassociative means that $S_{(\alpha, t)}$ is elliptic, as long as $t$ is sufficiently small.

Since $F_{2}(\alpha, t)$ depends smoothly on $t$ and $\hat{N}(t)$ is asymptotically coassociative near the singular points, we can apply the elliptic regularity theory from [19] to $S_{(\alpha, t)}$ as in the proof of Proposition 6.10. Moreover, as $E_{0}=E$ maps into $C_{\lambda-2}^{k-1, a}$ and $F_{2}$ varies smoothly with $t, E_{t}$ maps into $C_{\lambda-2}^{k-1, a}$ for $t$ sufficiently small. We can thus follow the proof of Proposition 6.10 to give the result. 


\subsection{Problem 3: moving singularities and varying $\mathbf{G}_{2}$ structure}

For our final problem, we consider CS deformations $N^{\prime}$ of $N$ as in Problem 2 , except now we allow $N^{\prime}$ to be coassociative under a deformation of the $\mathrm{G}_{2}$ structure on the ambient 7-manifold $M$.

Before we can define the moduli space, we need to choose a suitable family of nearby $\mathrm{G}_{2}$ structures to $(\varphi, g)$ on $M$. To achieve this end, we discuss the third cohomology of a tubular neighbourhood of $N$. Recall the notation from the start of this section and denote by $H_{\mathrm{cs}}^{m}(\hat{N})$ the $m$ th compactly supported cohomology of $\hat{N}$; that is, the quotient of closed compactly supported $m$-forms by the derivatives of compactly supported $(m-1)$-forms.

Proposition 6.19. Use the notation of Proposition 6.4. Let

$$
T=\hat{T} \cup \bigcup_{i=1}^{s} V_{i} \supseteq N .
$$

By making $\hat{T}$ and $V_{i}$, for $i=1, \ldots, s$, smaller if necessary, $T$ retracts onto $N$. There exists an isomorphism $\Xi: H_{\mathrm{dR}}^{3}(T) \rightarrow H_{\mathrm{cs}}^{3}(\hat{N})$.

Proof. Let $[\xi] \in H_{\mathrm{dR}}^{3}(T)$ and use the notation from the start of this section. Since $V_{i}$ retracts onto $\left\{z_{i}\right\}$ for $i=1, \ldots, s, \xi$ can be chosen such that $\left.\xi\right|_{V_{i}}=0$. Therefore, $\left.\xi\right|_{U_{i} \backslash\left\{z_{i}\right\}}=0$ which implies that the support of $\left.\xi\right|_{\hat{N}}$ is contained in $K$, which is compact. Hence $\left[\left.\xi\right|_{\hat{N}}\right]$ is a well-defined element of $H_{\mathrm{cs}}^{3}(\hat{N})$.

Define $\Xi$ by $[\xi] \mapsto\left[\left.\xi\right|_{\hat{N}}\right]$. We show that $\Xi$ is well defined. Suppose that $\xi^{\prime}=\xi+d v$, for $v \in C^{\infty}\left(\Lambda^{2} T^{*} T\right)$, such that $\left.\xi^{\prime}\right|_{V_{i}}=0$ for all $i$. Then $\left.d v\right|_{V_{i}}=0$ for all $i$. Since $V_{i}$ retracts onto $\left\{z_{i}\right\}$, we can choose $v$ such that $\left.v\right|_{V_{i}}=$ 0 without affecting $d v$ by smoothly interpolating over $\hat{T}$. Thus, $\left.v\right|_{\hat{N}}$ is compactly supported and $\left.\xi\right|_{\hat{N}}+d\left(\left.v\right|_{\hat{N}}\right)=\left.\xi^{\prime}\right|_{\hat{N}}$. Hence $\Xi$ is well-defined and injective.

Any closed form on $\hat{N}$ with support in $K$ can be extended smoothly to a closed form on $T$ which vanishes on $V_{i}$ for all $i$. Thus, any cohomology class in $H_{\mathrm{cs}}^{3}(\hat{N})$ has a representative $\gamma$ that can be lifted to a form $\xi$ on $T$ such that $\Xi([\xi])=[\gamma]$, which implies that $\Xi$ is surjective.

Notes. The reason for this result is 2 -fold.

(a) The condition $\Xi\left(\left[\left.\varphi\right|_{T}\right]\right)=0$ in $H_{\mathrm{cs}}^{3}(\hat{N})$ is implied by the coassociativity of $\hat{N}$ and it forces $\left[\left.\varphi\right|_{\hat{N}}\right]=0$ in $H_{\mathrm{cs}}^{3}(\hat{N})$. This is stronger than the seemingly more natural condition of $\left[\left.\varphi\right|_{\hat{N}}\right]=0$ in $H_{\mathrm{dR}}^{3}(\hat{N})$, which would be the correct requirement if $\hat{N}$ were compact by the work of McLean [17]. 
(b) If a $\mathrm{G}_{2}$ structure $\left(\varphi^{\prime}, g^{\prime}\right)$ on $M$ is such that $\Xi\left(\left[\left.\varphi^{\prime}\right|_{T}\right]\right) \neq 0$, then $\left.\varphi^{\prime}\right|_{\hat{N}^{\prime}} \neq 0$ for any deformation $\hat{N}^{\prime}$ of $\hat{N}$ in $T$, so there are no coassociative deformations.

From these observations, it is clear that Proposition 6.19 allows us to define a suitable distinguished family of "nearby" $\mathrm{G}_{2}$ structures to $(\varphi, g)$.

Definition 6.20. Let $\hat{\mathcal{F}}$ be a small open ball about 0 in $\mathbb{R}^{m}$ for some $m$ and let $\Xi$ be given by Proposition 6.19. Let

$$
\mathcal{F}=\left\{\left(\varphi^{f}, g^{f}\right): f \in \hat{\mathcal{F}}\right\}
$$

be a smooth family of torsion-free $\mathrm{G}_{2}$ structures, with $\left(\varphi^{0}, g^{0}\right)=(\varphi, g)$, such that $\Xi\left(\left[\left.\varphi^{f}\right|_{T}\right]\right)=0$ in $H_{\text {cs }}^{3}(\hat{N})$ and the map $h_{\hat{\mathcal{F}}}: \hat{\mathcal{F}} \rightarrow \mathcal{F}$ given by $h_{\hat{\mathcal{F}}}(f)=$ $\left(\varphi^{f}, g^{f}\right)$ is an embedding.

This is again notation which we shall use for the rest of the paper.

Note. $\hat{\mathcal{F}}$ can be considered as an open subset of $T_{0} \hat{\mathcal{F}}$.

We are now able to define the moduli space for Problem 3.

Definition 6.21. The moduli space of deformations $\mathcal{M}_{3}(N, \lambda)$ for Problem 3 is the set of pairs $\left(N^{\prime}, f\right)$ of $f \in \hat{\mathcal{F}}$ and $N^{\prime}$ in $\left(M, \varphi^{f}, g^{f}\right)$ which are CS coassociative 4 -folds at $z_{1}^{\prime}, \ldots, z_{s}^{\prime}$ with rate $\lambda$, having cone $C_{i}$ and tangent cone $\hat{C}_{i}^{\prime}$ at $z_{i}^{\prime}$ for all $i$, such that there exists a diffeomorphism $h: M \rightarrow M$, isotopic to the identity, such that $h\left(z_{i}\right)=z_{i}^{\prime}$ for $i=1, \ldots, s,\left.h\right|_{N}: N \rightarrow N^{\prime}$ is a homeomorphism and $\left.h\right|_{\hat{N}}: \hat{N} \rightarrow N^{\prime} \backslash\left\{z_{1}^{\prime}, \ldots, z_{s}^{\prime}\right\}$ is a diffeomorphism.

We have a projection map $\pi_{\hat{\mathcal{F}}}: \mathcal{M}_{3}(N, \lambda) \rightarrow \hat{\mathcal{F}}$, with $\pi_{\hat{\mathcal{F}}}\left(N^{\prime}, f\right)=f$, whose fibres $\pi_{\hat{\mathcal{F}}}^{-1}(f)$ are equal to the moduli space for Problem 2 defined using the $\mathrm{G}_{2}$ structure $\left(\varphi^{f}, g^{f}\right)$.

To incorporate the variation of the singular points and tangent ones, one might naively take the product of the translation space $\mathcal{T}$ with $\mathcal{F}$, then embed $\hat{\mathcal{T}} \times \hat{\mathcal{F}}$ into it in the obvious way. Unfortunately, the changing $\mathrm{G}_{2}$ structure means we must adapt $\mathcal{T}$ before we proceed. This is tackled in the next definition.

Definition 6.22. Use the notation of Definitions 6.12 and 6.20. For $f \in \hat{\mathcal{F}}$ and $\mathbf{z}^{\prime} \in B$, let $I^{f}\left(\mathbf{z}^{\prime}\right)$ denote the family of choices of $s$-tuples $\boldsymbol{\zeta}^{\prime}=\left(\zeta_{1}^{\prime}, \ldots, \zeta_{s}^{\prime}\right)$ of isomorphisms $\zeta_{i}^{\prime}: \mathbb{R}^{7} \rightarrow T_{z_{i}^{\prime}} M$ identifying $\left(\varphi_{0}, g_{0}\right)$ with $\left(\left.\varphi^{f}\right|_{T_{z_{i}^{\prime}} M},\left.g^{f}\right|_{T_{z_{i}^{\prime}} M}\right)$. 
The translation space corresponding to $\hat{\mathcal{F}}$ is

$$
\mathcal{T}^{\hat{\mathcal{F}}}=\left\{\left(\mathbf{z}^{\prime}, \boldsymbol{\zeta}^{\prime}, f\right): \mathbf{z}^{\prime} \in B, f \in \hat{\mathcal{F}}, \boldsymbol{\zeta}^{\prime} \in I^{f}\left(\mathbf{z}^{\prime}\right)\right\}
$$

It is a principal $\mathrm{G}_{2}^{s}$ bundle over $B \times \hat{\mathcal{F}}$.

There is a natural free action of $\mathrm{H}$ on $\mathcal{T}^{\hat{\mathcal{F}}}$ and hence an H-orbit through $(\mathbf{z}, \boldsymbol{\zeta}, 0)$. Therefore, we may embed $\hat{\mathcal{T}} \times \hat{\mathcal{F}}$ into $\mathcal{T}^{\hat{\mathcal{F}}}$ by $h_{\hat{\mathcal{T}} \times \hat{\mathcal{F}}}:(t, f) \mapsto$ $(\mathbf{z}(t, f), \boldsymbol{\zeta}(t, f), f)$ such that $h_{\hat{\mathcal{T}} \times \hat{\mathcal{F}}}(\hat{\mathcal{T}} \times \hat{\mathcal{F}})$ is transverse to this H-orbit, $h_{\hat{\mathcal{T}} \times \hat{\mathcal{F}}}(t, 0)=h_{\hat{\mathcal{T}}}(t)$ for all $t$ and $\mathbf{z}(0, f)=\mathbf{z}$ for all $f$.

Use the notation introduced before Proposition 6.13. Extend the $\mathrm{G}_{2}$ coordinate system used to define $N$ to a smooth family of $\mathrm{G}_{2}$ coordinate systems

$$
\left\{\left\{\chi_{i}(t, f): B(0 ; \eta) \longrightarrow V_{i}(t, f): i=1, \ldots, s\right\}:(t, f) \in \hat{\mathcal{T}} \times \hat{\mathcal{F}}\right\}
$$

such that $V_{i}(t, f)$ is an open set in $M$ containing $z_{i}(t, f), \chi_{i}(t, f)(0)=$ $z_{i}(t, f),\left.d \chi_{i}(t, f)\right|_{0}=\zeta_{i}(t, f), \chi_{i}(t, 0)=\chi_{i}(t), V_{i}(0, f)=V_{i}$ and $V_{i}(t, 0)=V_{i}(t)$ for $i=1, \ldots, s$. This extends the family of $\mathrm{G}_{2}$ coordinate systems introduced for Problem 2. Furthermore, the conditions $z_{i}(0, f)=z_{i}$ and $V_{i}(0, f)=V_{i}$ are natural because $(0, f)$ corresponds to the moduli space for Problem 1 in $\left(M, \varphi^{f}, g^{f}\right)$. We cannot expect $\zeta_{i}(0, f)$ to equal $\zeta_{i}$ because they identify different $G_{2}$ structures with the standard $G_{2}$ structure on $\mathbb{R}^{7}$. This trend continues in the statement of the next result, which is the analogue of Proposition 6.13.

Proposition 6.23. Use the notation from the start of this section, from Propositions 6.4 and 6.13, and from Definition 6.22 .

(a) There exists a family $\mathcal{N}^{\hat{\mathcal{F}}}=\{N(t, f):(t, f) \in \hat{\mathcal{T}} \times \hat{\mathcal{F}}\}$ of $C S$ 4-folds in $M$, with $N(0, f)=N$ and $N(t, 0)=N(t)$, such that $N(t, f)$ has a singularity at $z_{i}(t, f)$, for $i=1, \ldots, s$, with rate $\lambda$, cone $C_{i}$ and tangent cone $\hat{C}_{i}(t, f)=\left(\zeta_{i}(t, f) \circ \iota_{i}\right)\left(C_{i}\right)$.

(b) Let $\hat{N}(t, f)=N(t, f) \backslash\left\{z_{1}(t, f), \ldots, z_{s}(t, f)\right\}$ and write $N(t, f)=$ $K(t, f) \sqcup \bigsqcup_{i=1}^{s} U_{i}(t, f)$, where $K(t, f)$ is compact and $U_{i}(t, f) \backslash$ $\left\{z_{i}(t, f)\right\} \cong(0, \epsilon) \times \Sigma_{i}$ for all $i$, in the obvious way, ensuring that $K(0, f)=K, K(t, 0)=K(t), U_{i}(0, f)=U_{i}$ and $U_{i}(t, 0)=U_{i}(t)$.

For $(t, f) \in \hat{\mathcal{T}} \times \hat{\mathcal{F}}$, there exist open sets $\hat{T}(t, f) \subseteq M$ containing $\hat{N}(t, f)$ and diffeomorphisms $\delta(t, f): \hat{U} \rightarrow \hat{T}(t, f)$ taking the zero section to $\hat{N}(t, f)$, varying smoothly in $t$ and $f$, with $\hat{T}(0, f)=\hat{T}$, 
$\hat{T}(t, 0)=\hat{T}(t)$ and $\delta(t, 0)=\delta(t)$. Moreover, $\delta(t, f)$ is compatible with the identifications $U_{i}(t, f) \backslash\left\{z_{i}(t, f)\right\} \cong(0, \epsilon) \times \Sigma_{i}$ for $i=1, \ldots, s$.

The proof is almost identical to that of Proposition 6.13 so we omit it. The compatibility conditions on $\delta(t, f)$ are given by commutative diagrams like (6.16).

Remark. $\delta(t, f)$ is not required to be compatible with the isomorphism $\nu(\hat{N}) \cong \Lambda_{+}^{2} T^{*} \hat{N}$ for $(t, f) \neq(0,0)$.

We proceed by defining our final deformation map.

Definition 6.24. Use the notation of Definition 6.5 and Proposition 6.23. For $(t, f) \in \hat{\mathcal{T}} \times \hat{\mathcal{F}}$, let $f_{\alpha}(t, f)=\delta(t, f) \circ \pi_{\alpha}, \hat{N}_{\alpha}(t, f)=f_{\alpha}(t, f)(\hat{N})$ and $N_{\alpha}(t, f)=\hat{N}_{\alpha}(t, f) \cup\left\{z_{1}(t, f), \ldots, z_{s}(t, f)\right\}$. Define $F_{3}$ on $C_{\text {loc }}^{1}(\hat{U}) \times \hat{\mathcal{T}} \times$ $\hat{\mathcal{F}}$ by:

$$
F_{3}(\alpha, t, f)=f_{\alpha}(t, f)^{*}\left(\left.\varphi^{f}\right|_{\hat{N}_{\alpha}(t, f)}\right) \in C_{\mathrm{loc}}^{0}\left(\Lambda^{3} T^{*} \hat{N}\right) .
$$

The linearization of $F_{3}$ at $(0,0,0)$ acts as

$$
\left.d F_{3}\right|_{(0,0,0)}:(\alpha, t, f) \longmapsto d \alpha+L_{2}(t)+L_{3}(f),
$$

where $\alpha \in C_{\text {loc }}^{1}\left(\Lambda_{+}^{2} T^{*} \hat{N}\right),(t, f) \in T_{0} \hat{\mathcal{T}} \oplus T_{0} \hat{\mathcal{F}}, L_{2}$ is given in Definition 6.14 and $L_{3}$ is a linear map into the space of smooth exact 3-forms on $\hat{N}$ by the condition imposed on $\varphi^{f}$ in Definition 6.20.

Note. $F_{3}(\alpha, t, 0)=F_{2}(\alpha, t)$ as given in Definition 6.14.

Clearly, Ker $F_{3}$ corresponds to $\hat{N}_{\alpha}(t, f)$ on which $\varphi^{f}$ vanishes, and we have the analogue of Proposition 6.15 by considering its proof.

Proposition 6.25. In the notation of Definitions 6.20 and $6.24, \mathcal{M}_{3}(N, \lambda)$ is locally homeomorphic to $\operatorname{Ker} F_{3}=\left\{(\alpha, t, f) \in C_{\lambda}^{\infty}(\hat{U}) \times \hat{\mathcal{T}} \times \hat{\mathcal{F}}\right.$ : $\left.F_{3}(\alpha, t, f)=0\right\}$.

We again associate a map to our deformation map.

Definition 6.26. Use the notation of Definition 6.24. Define $G_{3}: C_{\text {loc }}^{1}(\hat{U}) \times$ $C_{\text {loc }}^{1}\left(\Lambda^{4} T^{*} \hat{N}\right) \times \hat{\mathcal{T}} \times \hat{\mathcal{F}} \rightarrow C_{\text {loc }}^{0}\left(\Lambda^{3} T^{*} \hat{N}\right)$ by

$$
G_{3}(\alpha, \beta, t, f)=F_{3}(\alpha, t, f)+d^{*} \beta,
$$

so that $\left.d G_{3}\right|_{(0,0,0,0)}:(\alpha, \beta, t, f) \longmapsto d \alpha+d^{*} \beta+L_{2}(t)+L_{3}(f)$. 
The next result is analogous to Propositions 6.8 and 6.17 and may be immediately deduced from the exactness of $F_{3}(\alpha, t, f)$, which follows from the choice of $\varphi^{f}$ in Definition 6.20.

\section{Proposition 6.27.}

$\operatorname{Ker} F_{3} \cong\left\{(\alpha, \beta, t, f) \in C_{\lambda}^{\infty}(\hat{U}) \times C_{\lambda}^{\infty}\left(\Lambda^{4} T^{*} \hat{N}\right) \times \hat{\mathcal{T}} \times \hat{\mathcal{F}}: G_{3}(\alpha, \beta, t, f)=0\right\}$

The argument used to prove the regularity result Proposition 6.18 is easily generalized to the map $G_{3}$, so we end the section with the following.

Proposition 6.28. Let $(\alpha, \beta, t, f) \in L_{k+1, \lambda}^{p}(\hat{U}) \times L_{k+1, \lambda}^{p}\left(\Lambda^{4} T^{*} \hat{N}\right) \times \hat{\mathcal{T}} \times \hat{\mathcal{F}}$, where $p>4$ and $k \geq 2$. If $G_{3}(\alpha, \beta, t, f)=0$, where $G_{3}$ is given in Definition 6.26 , and $\|\alpha\|_{C_{1}^{1}}$, $t$ and $f$ are sufficiently small, $(\alpha, \beta) \in C_{\lambda}^{\infty}(\hat{U}) \times$ $C_{\lambda}^{\infty}\left(\Lambda^{4} T^{*} \hat{N}\right)$.

Note. We are free to make $\hat{\mathcal{F}}$ smaller, so we can make $f$ as small as necessary.

\section{The deformation and obstruction spaces}

The scheme we use in this section, for each of our deformation problems in turn, is the following. First we get an analytic description of the infinitesimal deformation and obstruction spaces: the deformation space is the kernel of the linearization of the deformation map at zero, and the obstruction space is a distinguished subspace of the cokernel of the linearization, both in suitable weighted Banach spaces. We then use the implicit function theorem to show that the set of self-dual 2-forms whose image under the deformation map lies in the obstruction space is locally diffeomorphic to the infinitesimal deformation space. Finally, since the moduli space is locally characterized as the kernel of the deformation map, the projection from this set of self-dual 2 -forms to the obstruction space is a smooth map between smooth manifolds whose fibre at zero is locally homeomorphic to the moduli space.

We recollect the common notation introduced in $\S 6$, particularly at the start. In addition, fix some $p>4$ and integer $k \geq 2$ so that we can use the regularity results in $\S 6$ for the kernels of the deformation maps in $L_{k+1, \lambda}^{p}$.

\subsection{Problem 1}

Recall the maps $F_{1}$ and $G_{1}$ given in Definitions 6.5 and 6.7, respectively. Their kernels, which are isomorphic, give a local description for the moduli 
space $\mathcal{M}_{1}(N, \lambda)$ by Propositions 6.6 and 6.8. Therefore the kernels of $\left.d F_{1}\right|_{0}$ and $\left.d G_{1}\right|_{(0,0)}$ describe the infinitesimal deformations.

Definition 7.1. The infinitesimal deformation space for Problem 1 is

$$
\mathcal{I}_{1}(N, \lambda)=\left\{(\alpha, \beta) \in L_{k+1, \lambda}^{p}\left(\Lambda_{+}^{2} T^{*} \hat{N} \oplus \Lambda^{4} T^{*} \hat{N}\right): d \alpha+d^{*} \beta=0\right\} .
$$

Therefore, $\mathcal{I}_{1}(N, \lambda)$ is finite-dimensional.

To see that this is a reasonable choice, Proposition 6.10 gives that

$$
\begin{aligned}
\mathcal{I}_{1}(N, \lambda) & =\left\{(\alpha, \beta) \in C_{\lambda}^{\infty}\left(\Lambda_{+}^{2} T^{*} \hat{N} \oplus \Lambda^{4} T^{*} \hat{N}\right): d \alpha+d^{*} \beta=0\right\} \\
& \cong\left\{\alpha \in C_{\lambda}^{\infty}\left(\Lambda_{+}^{2} T^{*} \hat{N}\right): d \alpha=0\right\}
\end{aligned}
$$

where the equivalence follows from the maximum principle.

We turn to possible obstructions and start by describing the image of $F_{1}$. The proof is long but the basic idea is that $\varphi$ is exact near $N$, so $F_{1}(\alpha)$ is the derivative of a 2 -form. The technical issue is that the 2 -form is not guaranteed to lie in the correct weighted Sobolev space. This is resolved by specifying its behaviour near the singularities and then considering the cokernel of $d+d^{*}$.

Proposition 7.2. In the notation of Proposition 6.4 and Definition 6.5, the map $F_{1}$ takes $L_{k+1, \lambda}^{p}(\hat{U})$ into $\overline{d\left(L_{k+1, \lambda}^{p}\left(\Lambda^{2} T^{*} \hat{N}\right)\right)} \subseteq L_{k, \lambda}^{p}\left(\Lambda^{3} T^{*} \hat{N}\right)$.

Remark. The closure is a technicality forced upon us because a certain map is not guaranteed to be Fredholm and so may not have closed image.

Proof. Let $\alpha \in L_{k+1, \lambda}^{p}(\hat{U})$, recall the notation from the start of $\S 6$ and let $T$ be as in Proposition 6.19. As noted after that proposition, $\left[\left.\varphi\right|_{T}\right]=0$ in $H_{\mathrm{dR}}^{3}(T)$ and hence $\left.\varphi\right|_{T}$ is exact. Thus, $\left.\varphi\right|_{T}=d \psi$ for some $\psi \in C^{\infty}\left(\Lambda^{2} T^{*} T\right)$. However, we want to select $\psi$ in a particular way near the singularities. On $B(0 ; \eta) \subseteq \mathbb{R}^{7}$

$$
\chi_{i}^{*}(\varphi)=\varphi_{0}+O\left(r_{i}\right)
$$

If $v$ is the dilation vector field on $\mathbb{R}^{7}$, given in coordinates $\left(x_{1}, \ldots, x_{7}\right)$ by

$$
v=x_{1} \frac{\partial}{\partial x_{1}}+\cdots+x_{7} \frac{\partial}{\partial x_{7}},
$$

then calculation shows that $d\left(v \cdot \varphi_{0}\right)=3 \varphi_{0}$. Thus, we can choose $\psi$ to satisfy

$$
\chi_{i}^{*}(\psi)=\frac{1}{3}\left(v \cdot \varphi_{0}\right)+O\left(r_{i}^{2}\right)
$$


on $V_{i}$, then extend $\psi$ smoothly to a form on $T$ such that $d \psi=\left.\varphi\right|_{T}$. Note that

$$
\left.\left(v \cdot \varphi_{0}\right)\right|_{\iota_{i}\left(C_{i}\right)}=v \cdot\left(\left.\varphi_{0}\right|_{\iota_{i}\left(C_{i}\right)}\right)=0
$$

as $v \in T\left(\iota_{i}\left(C_{i}\right)\right)$. Hence $\chi_{i}^{*}(\psi)=O\left(r_{i}^{2}\right)$ on $\iota_{i}\left(C_{i}\right)$, for all $i$, and similar results hold for the derivatives of $\psi$. In the notation of Definition 6.5, define

$$
H_{1}(\alpha)=f_{\alpha}^{*}\left(\left.\psi\right|_{\hat{N}_{\alpha}}\right)
$$

so that $F_{1}(\alpha)=d\left(H_{1}(\alpha)\right)$. Note that $\left.\chi_{i}^{*}(\psi)\right|_{\iota_{i}\left(C_{i}\right)}=O\left(r_{i}^{2}\right)$ is dominated by $O\left(r_{i}^{\lambda}\right)$ terms as $r_{i} \rightarrow 0$ since $\lambda<2$. Further, $H_{1}(\alpha)$ has the same growth as $\left.\chi_{i}^{*}(\psi)\right|_{\left(\Phi_{\alpha}\right)_{i}\left((0, \epsilon) \times \Sigma_{i}\right)}$ as $r_{i} \rightarrow 0$, using the notation preceding Proposition 6.6. Continuing to use the aforementioned notation, we see that

$$
\left.\chi_{i}^{*}(\psi)\right|_{\left(\Phi_{\alpha}\right)_{i}\left((0, \epsilon) \times \Sigma_{i}\right)}=\left.\chi_{i}^{*}(\psi)\right|_{\left(\left(\Phi_{\alpha}\right)_{i}-\iota_{i}\right)\left((0, \epsilon) \times \Sigma_{i}\right)}+\left.\chi_{i}^{*}(\psi)\right|_{\iota_{i}\left((0, \epsilon) \times \Sigma_{i}\right)} .
$$

The first term on the right-hand side depends on $\left|\left(\Phi_{\alpha}\right)_{i}-\iota_{i}\right|$ and hence is $O\left(r_{i}^{\lambda}\right)$ as $r_{i} \rightarrow 0$. This dominates the second term by our observation above. Hence, $H_{1}(\alpha) \in L_{k, \lambda}^{p}$ because $H_{1}$ depends on $\alpha$ and $\nabla \alpha$. Notice that $H_{1}(\alpha)$ has one degree of differentiability less than expected.

We deduce that $F_{1}(\alpha) \in d\left(L_{k, \lambda}^{p}\left(\Lambda^{2} T^{*} \hat{N}\right)\right)$ and so lies in the image of $d+d^{*}: L_{k, \lambda}^{p}\left(\Lambda^{2} T^{*} \hat{N} \oplus \Lambda^{4} T^{*} \hat{N}\right) \rightarrow L_{k-1, \lambda-1}^{p}\left(\Lambda^{3} T^{*} \hat{N}\right)$, where we include a projection to 3 -forms. Therefore, as the dual of a Sobolev space on $\hat{N}$ with weight $\mu$ has weight $-4-\mu, F_{1}(\alpha)$ is $L^{2}$-orthogonal to the kernel $\mathcal{K}^{3}$ of the adjoint map

$$
d+d^{*}: L_{l+1,-3-\lambda}^{q}\left(\Lambda^{3} T^{*} \hat{N}\right) \longrightarrow L_{l,-4-\lambda}^{q}\left(\Lambda^{2} T^{*} \hat{N} \oplus \Lambda^{4} T^{*} \hat{N}\right)
$$

where $q>1$ such that $1 / p+1 / q=1$ and $l \in \mathbb{N}$. (By elliptic regularity, we are free to choose any $l \in \mathbb{N}$ as $\mathcal{K}^{3}$ is independent of $l$.) We show below that

$$
\overline{d\left(L_{k, \lambda}^{p}\left(\Lambda^{2} T^{*} \hat{N}\right)\right)} \oplus d^{*}\left(L_{k, \lambda}^{p}\left(\Lambda^{4} T^{*} \hat{N}\right)\right) \subseteq L_{k-1, \lambda-1}^{p}\left(\Lambda^{3} T^{*} \hat{N}\right)
$$

is characterized as the subspace which is $L^{2}$-orthogonal to $\mathcal{K}^{3}$. Given this, as $\mathcal{K}^{3}$ is independent of $k$, the same holds with $k$ replaced by $k+1$. Since $F_{1}(\alpha) \in L_{k, \lambda-1}^{p}$, we deduce that $F_{1}(\alpha)$ is the limit of a sequence $d \alpha_{n}+d^{*} \beta$ for some $\left(\alpha_{n}, \beta\right) \in L_{k+1, \lambda}^{p}\left(\Lambda^{2} T^{*} \hat{N} \oplus \Lambda^{4} T^{*} \hat{N}\right)$. As $F_{1}(\alpha)$ is exact, the usual maximum principle argument applied to the harmonic 4 -form $\beta$ forces it to be zero and the result is proved.

Recall that $\lambda \notin \mathcal{D}$, so that the map (5.1), for $\mu=\lambda$, discussed in $\S 5$ is Fredholm. Therefore, its image, which is $d\left(L_{k, \lambda}^{p}\left(\Lambda_{+}^{2} T^{*} \hat{N}\right)\right) \oplus d^{*}\left(L_{k, \lambda}^{p}\right.$ 
$\left.\left(\Lambda^{4} T^{*} \hat{N}\right)\right)$ by the now familiar maximum principle argument, is closed, so each constituent part is closed as it is a direct sum. Consider

$$
d+d^{*}: L_{k, \lambda}^{p}\left(\Lambda^{\text {even }} T^{*} \hat{N}\right) \rightarrow L_{k-1, \lambda-1}^{p}\left(\Lambda^{\text {odd }} T^{*} \hat{N}\right) .
$$

This elliptic map has image whose closure comprises precisely of those elements of $L_{k-1, \lambda-1}^{p}\left(\Lambda^{\text {odd }} T^{*} \hat{N}\right)$ which are $L^{2}$-orthogonal to the kernel $\mathcal{K}$ of

$$
d+d^{*}: L_{l+1,-3-\lambda}^{q}\left(\Lambda^{\text {odd }} T^{*} \hat{N}\right) \rightarrow L_{l,-4-\lambda}^{q}\left(\Lambda^{\text {even }} T^{*} \hat{N}\right) .
$$

The space $\mathcal{K}$ can be written as the direct $\operatorname{sum} \mathcal{K}=\mathcal{K}^{1} \oplus \mathcal{K}^{3} \oplus \mathcal{K}^{m}$, where

$$
\mathcal{K}^{j}=\mathcal{K} \cap L_{l+1,-3-\lambda}^{q}\left(\Lambda^{j} T^{*} \hat{N}\right)
$$

for $j=1$ and 3 and $\mathcal{K}^{m}$ is some transverse subspace. Recall that, in $L_{k-1, \lambda-1}^{p}$, the image of $d^{*}$ on 4 -forms is closed, and exact and coexact forms meet only at zero by the maximum principle. Thus, the projection of the image of (7.1) to 3 -forms has closure

$\overline{d\left(L_{k, \lambda}^{p}\left(\Lambda^{2} T^{*} \hat{N}\right)\right)} \oplus d^{*}\left(L_{k, \lambda}^{p}\left(\Lambda^{4} T^{*} \hat{N}\right)\right)=\left\{\alpha_{3}: \exists \alpha_{1}\right.$ such that $\left.\left(\alpha_{1}, \alpha_{3}\right) \in \mathcal{K}^{\perp}\right\}$.

Note that the projection $\pi_{1}\left(\mathcal{K}^{m}\right)$ of $\mathcal{K}^{m}$ onto the space of 1 -forms must meet $\mathcal{K}^{1}$ in the zero form since, if $\left(\alpha_{1}, \alpha_{3}\right) \in \mathcal{K}^{m}$ and $\alpha_{1} \in \mathcal{K}^{1}$, then $\alpha_{3} \in \mathcal{K}^{3}$, which contradicts the direct sum decomposition of $\mathcal{K}$. Therefore, $\pi_{1}\left(\mathcal{K}^{m}\right)$ and $\mathcal{K}^{1}$ are transverse finite-dimensional subspaces of $L_{l+1,-3-\lambda}^{q}\left(\Lambda^{1} T^{*} \hat{N}\right)$. Hence, there exists a space $\mathcal{A}$ of smooth compactly supported 1 -forms on $\hat{N}$ which is $L^{2}$-orthogonal to $\mathcal{K}^{1}$ and such that $\mathcal{A} \times \mathcal{K}^{m} \rightarrow \mathbb{R}$ given by $(\gamma, \xi) \mapsto(\gamma, 0) \cdot \xi$ is a dual pairing. If $\alpha_{3} \in L_{k-1, \lambda-1}^{p}\left(\Lambda^{3} T^{*} \hat{N}\right)$ such that $\alpha_{3} \in\left(\mathcal{K}^{3}\right)^{\perp}$, there exists a unique $\alpha_{1} \in \mathcal{A}$ such that $\left(\alpha_{1}, 0\right) \cdot \xi=-\left(0, \alpha_{3}\right) \cdot \xi$ for all $\xi \in \mathcal{K}^{m}$, which implies that $\left(\alpha_{1}, \alpha_{3}\right) \in\left(\mathcal{K}^{m}\right)^{\perp}$. We conclude that

$$
\begin{aligned}
\left(\mathcal{K}^{3}\right)^{\perp} & =\left\{\alpha_{3} \in\left(\mathcal{K}^{3}\right)^{\perp}: \exists \alpha_{1} \in\left(\mathcal{K}^{1}\right)^{\perp} \text { such that }\left(\alpha_{1}, \alpha_{3}\right) \in\left(\mathcal{K}^{m}\right)^{\perp}\right\} \\
& =\left\{\alpha_{3}: \exists \alpha_{1} \text { such that }\left(\alpha_{1}, \alpha_{3}\right) \in \mathcal{K}^{\perp}\right\} \\
& =\overline{d\left(L_{k, \lambda}^{p}\left(\Lambda^{2} T^{*} \hat{N}\right)\right)} \oplus d^{*}\left(L_{k, \lambda}^{p}\left(\Lambda^{4} T^{*} \hat{N}\right)\right) \subseteq L_{k-1, \lambda-1}^{p}\left(\Lambda^{3} T^{*} \hat{N}\right)
\end{aligned}
$$

as required. 
We deduce from Propositions $6.6,6.8,6.10$ and 7.2 that $\mathcal{M}_{1}(N, \lambda)$ is locally homeomorphic to the kernel of

$$
\begin{aligned}
G_{1}: L_{k+1, \lambda}^{p}(\hat{U}) \times \frac{L_{k+1, \lambda}^{p}\left(\Lambda^{4} T^{*} \hat{N}\right)}{d\left(L_{k+1, \lambda}^{p}\left(\Lambda^{2} T^{*} \hat{N}\right)\right)} \oplus d^{*}\left(L_{k+1, \lambda}^{p}\left(\Lambda^{4} T^{*} \hat{N}\right)\right) \subseteq L_{k, \lambda-1}^{p}\left(\Lambda^{3} T^{*} \hat{N}\right) & .
\end{aligned}
$$

By the implicit function theorem (Theorem 4.5), the regularity result Proposition 6.10 and Definition 7.1, if the target space of $G_{1}$ equals the image of

$$
\begin{aligned}
\left.d G_{1}\right|_{(0,0)}: L_{k+1, \lambda}^{p}\left(\Lambda_{+}^{2} T^{*} \hat{N} \oplus \Lambda^{4} T^{*} \hat{N}\right) & \longrightarrow L_{k, \lambda-1}^{p}\left(\Lambda^{3} T^{*} \hat{N}\right) \\
(\alpha, \beta) & \longmapsto d \alpha+d^{*} \beta,
\end{aligned}
$$

then $\mathcal{M}_{1}(N, \lambda)$ is a smooth manifold of dimension $\operatorname{dim} \mathcal{I}(N, \lambda)$. Therefore, our deformation theory will be obstructed if and only if the map

$$
d: L_{k+1, \lambda}^{p}\left(\Lambda_{+}^{2} T^{*} \hat{N}\right) \longrightarrow \overline{d\left(L_{k+1, \lambda}^{p}\left(\Lambda^{2} T^{*} \hat{N}\right)\right)}
$$

is not surjective. This leads us to the next result and definition.

Proposition 7.3. There exists a finite-dimensional subspace $\mathcal{O}_{1}(N, \lambda)$ of $L_{k, \lambda-1}^{p}\left(\Lambda^{3} T^{*} \hat{N}\right)$ such that

$$
\overline{d\left(L_{k+1, \lambda}^{p}\left(\Lambda^{2} T^{*} \hat{N}\right)\right)}=d\left(L_{k+1, \lambda}^{p}\left(\Lambda_{+}^{2} T^{*} \hat{N}\right)\right) \oplus \mathcal{O}_{1}(N, \lambda)
$$

Proof. As noted in the proof of Proposition 7.2, the Fredholmness of (5.1) for $\mu=\lambda \notin \mathcal{D}$ implies that each part of $d\left(L_{k+1, \lambda}^{p}\left(\Lambda_{+}^{2} T^{*} \hat{N}\right)\right) \oplus d^{*}\left(L_{k+1, \lambda}^{p}\right.$ $\left.\left(\Lambda^{4} T^{*} \hat{N}\right)\right)$ is closed. Moreover, the sum has finite codimension in $L_{k, \lambda-1}^{p}$ $\left(\Lambda^{3} T^{*} \hat{N}\right)$. Since

$$
\begin{aligned}
d\left(L_{k+1, \lambda}^{p}\left(\Lambda_{+}^{2} T^{*} \hat{N}\right)\right) & \oplus d^{*}\left(L_{k+1, \lambda}^{p}\left(\Lambda^{4} T^{*} \hat{N}\right)\right) \\
& \subseteq \overline{d\left(L_{k+1, \lambda}^{p}\left(\Lambda^{2} T^{*} \hat{N}\right)\right)} \oplus d^{*}\left(L_{k+1, \lambda}^{p}\left(\Lambda^{4} T^{*} \hat{N}\right)\right),
\end{aligned}
$$

the latter has finite codimension in $L_{k, \lambda-1}^{p}\left(\Lambda^{3} T^{*} \hat{N}\right)$, and the former has finite codimension in the latter. Thus, $\mathcal{O}_{1}(N, \lambda)$ can be chosen as stated.

Definition 7.4. The obstruction space for Problem 1 is

$$
\mathcal{O}_{1}(N, \lambda) \cong \frac{\overline{d\left(L_{k+1, \lambda}^{p}\left(\Lambda^{2} T^{*} \hat{N}\right)\right)}}{d\left(L_{k+1, \lambda}^{p}\left(\Lambda_{+}^{2} T^{*} \hat{N}\right)\right)}
$$


We now have all the ingredients necessary to apply the implicit function theorem. Use the notation introduced in $\S 6$ and this section. Define

$$
\begin{aligned}
& U_{1}=L_{k+1, \lambda}^{p}(\hat{U}) \times L_{k+1, \lambda}^{p}\left(\Lambda^{4} T^{*} \hat{N}\right), \\
& X_{1}=L_{k+1, \lambda}^{p}\left(\Lambda_{+}^{2} T^{*} \hat{N} \oplus \Lambda^{4} T^{*} \hat{N}\right), \\
& Y_{1}=\mathcal{O}_{1}(N, \lambda) \subseteq L_{k, \lambda-1}^{p}\left(\Lambda^{3} T^{*} \hat{N}\right) \text { and } \\
& Z_{1}=\overline{d\left(L_{k+1, \lambda}^{p}\left(\Lambda^{2} T^{*} \hat{N}\right)\right)} \oplus d^{*}\left(L_{k+1, \lambda}^{p}\left(\Lambda^{4} T^{*} \hat{N}\right)\right) \subseteq L_{k, \lambda-1}^{p}\left(\Lambda^{3} T^{*} \hat{N}\right) .
\end{aligned}
$$

Then $X_{1}, Y_{1}$ and $Z_{1}$ are Banach spaces: $X_{1}$ by definition, $Y_{1}$ because it is a finite-dimensional subspace of a Banach space and $Z_{1}$ because it is a closed subspace of a Banach space as noted in the proof of Proposition 7.2. Further, $U_{1}$ is an open neighbourhood of $(0,0)$ in $X_{1}$ because $L_{k+1, \lambda}^{p} \hookrightarrow C_{1}^{1}$ by Theorem 4.4 and $\hat{U}$ is an open subset of $C_{1}^{1}$ by Proposition 6.4. Thus, $W_{1}=U_{1} \times Y_{1}$ is an open neighbourhood of $((0,0), 0)$ in $X_{1} \times Y_{1}$. Define $\mathcal{G}_{1}: W_{1} \rightarrow Z_{1}$ by:

$$
\mathcal{G}_{1}((\alpha, \beta), \gamma)=G_{1}(\alpha, \beta)+\gamma
$$

Then $\mathcal{G}_{1}$ is well-defined by Propositions 7.2 and 7.3 and its derivative at $((0,0), 0)$ acts surjectively from $X_{1} \times Y_{1}$ to $Z_{1}$ as

$$
\left.d \mathcal{G}_{1}\right|_{((0,0), 0)}:((\alpha, \beta), \gamma) \longmapsto d \alpha+d^{*} \beta+\gamma
$$

Using Definition 7.1 and the fact that $\left(d+d^{*}\right)\left(X_{1}\right) \cap Y_{1}=\{0\}$, we see that

$$
\begin{aligned}
\left.\operatorname{Ker} d \mathcal{G}_{1}\right|_{((0,0), 0)} & =\left\{((\alpha, \beta), \gamma) \in X_{1} \times Y_{1}: d \alpha+d^{*} \beta+\gamma=0\right\} \\
& \cong\left\{(\alpha, \beta) \in X_{1}: d \alpha+d^{*} \beta=0\right\}=\mathcal{I}_{1}(N, \lambda)
\end{aligned}
$$

The conclusion, by implementing the implicit function theorem (Theorem 4.5 ), is that $\operatorname{Ker} \mathcal{G}_{1}$ is a smooth manifold near zero locally diffeomorphic to an open neighbourhood $\hat{\mathcal{M}}_{1}(N, \lambda)$ of $(0,0)$ in $\mathcal{I}_{1}(N, \lambda)$. Formally, if we write $X_{1}=\mathcal{I}_{1}(N, \lambda) \oplus A$ for some closed subspace $A$ of $X_{1}$, there exist open sets $\hat{\mathcal{M}}_{1}(N, \lambda) \subseteq \mathcal{I}_{1}(N, \lambda), V_{A} \subseteq A, V_{Y} \subseteq Y_{1}$, all containing zero, with $\hat{\mathcal{M}}_{1}(N, \lambda) \times V_{A} \subseteq U_{1}$, and smooth maps $\mathcal{V}_{A}: \hat{\mathcal{M}}_{1}(N, \lambda) \rightarrow V_{A}$ and $\mathcal{V}_{Y}$ : $\hat{\mathcal{M}}_{1}(N, \lambda) \rightarrow V_{Y}$ such that

$\operatorname{Ker} \mathcal{G}_{1} \cap\left(\left(\hat{\mathcal{M}}_{1}(N, \lambda) \times V_{A}\right) \times V_{Y}\right)=\left\{\left(\left(x, \mathcal{V}_{A}(x)\right), \mathcal{V}_{Y}(x)\right): x \in \hat{\mathcal{M}}_{1}(N, \lambda)\right\}$

Define a smooth map $\pi_{1}: \hat{\mathcal{M}}_{1}(N, \lambda) \rightarrow \mathcal{O}_{1}(N, \lambda)$ by $\pi_{1}(x)=\mathcal{V}_{Y}(x)$. Its kernel is locally homeomorphic to the kernel of $G_{1}$ and it can be considered as 
a projection from a subset of the infinitesimal deformation space onto the obstruction space. Hence, the moduli space $\mathcal{M}_{1}(N, \lambda)$ near $N$ is homeomorphic to the kernel of $\pi_{1}$ near 0 . We write this result as a theorem.

Theorem 7.5. Use the notation of Definitions 6.1, 7.1 and 7.4. There exist a smooth manifold $\hat{\mathcal{M}}_{1}(N, \lambda)$, which is an open neighbourhood of $(0,0)$ in $\mathcal{I}_{1}(N, \lambda)$, and a smooth map $\pi_{1}: \hat{\mathcal{M}}_{1}(N, \lambda) \rightarrow \mathcal{O}_{1}(N, \lambda)$, with $\pi_{1}(0,0)=0$, such that an open neighbourhood of 0 in $\operatorname{Ker} \pi_{1}$ is homeomorphic to an open neighbourhood of $N$ in $\mathcal{M}_{1}(N, \lambda)$.

We deduce that, if the obstruction space is zero, the moduli space for Problem 1 is a smooth manifold near $N$ of dimension equal to that of the infinitesimal deformation space. We expect the obstruction space to be zero for generic choices of $N$ and the $\mathrm{G}_{2}$ structure on $M$.

\subsection{Problem 2}

Recall the notation introduced in Definitions 6.12, 6.14 and 6.16. We begin by defining the infinitesimal deformation space for this problem.

Definition 7.6. The infinitesimal deformation space for Problem 2 is

$$
\begin{aligned}
& \mathcal{I}_{2}(N, \lambda) \\
& \quad=\left\{(\alpha, \beta, t) \in L_{k+1, \lambda}^{p}\left(\Lambda_{+}^{2} T^{*} \hat{N} \oplus \Lambda^{4} T^{*} \hat{N}\right) \oplus T_{0} \hat{\mathcal{T}}: d \alpha+d^{*} \beta+L_{2}(t)=0\right\},
\end{aligned}
$$

which is finite-dimensional.

Using Proposition 6.18 and the maximum principle,

$$
\begin{aligned}
\mathcal{I}_{2}(N, \lambda)= & \left\{(\alpha, \beta, t) \in C_{\lambda}^{\infty}\left(\Lambda_{+}^{2} T^{*} \hat{N} \oplus \Lambda^{4} T^{*} \hat{N}\right) \oplus T_{0} \hat{\mathcal{T}}: d \alpha\right. \\
& \left.+d^{*} \beta+L_{2}(t)=0\right\} \\
\cong & \left\{(\alpha, t) \in C_{\lambda}^{\infty}\left(\Lambda_{+}^{2} T^{*} \hat{N}\right) \oplus T_{0} \hat{\mathcal{T}}: d \alpha+L_{2}(t)=0\right\}
\end{aligned}
$$

since $d \alpha+L_{2}(t)$ is exact by Definition 6.14 .

Note. There is a subspace of $\mathcal{I}_{2}(N, \lambda)$ which is isomorphic to $\mathcal{I}_{1}(N, \lambda)$.

To start our consideration of obstructions, we have the easy generalization of Proposition 7.2.

Proposition 7.7. The map $F_{2}$ given in Definition 6.14 takes $L_{k+1, \lambda}^{p}(\hat{U}) \times$ $\hat{\mathcal{T}}$ into $\overline{d\left(L_{k+1, \lambda}^{p}\left(\Lambda^{2} T^{*} \hat{N}\right)\right)} \subseteq L_{k, \lambda-1}^{p}\left(\Lambda^{3} T^{*} \hat{N}\right)$. 
Proof. Use the notation from Proposition 6.13 and its proof and from the proof of Proposition 7.2. Recall that we have an open set $T \supseteq \hat{T}$ in $M$ containing $N$, which retracts onto $N$, and $\psi \in C^{\infty}\left(\Lambda^{2} T^{*} T\right)$ such that $d \psi=\left.\varphi\right|_{T}$.

Let $T(t)=\hat{T}(t) \cup \bigcup_{i=1}^{s} V_{i}(t)$. By making $\hat{T}(t)$ and $V_{i}(t)$ smaller if necessary, $T(t)$ is an open set in $M$, varying smoothly in $t$, which retracts onto $N$. We may choose $\psi(t)$ to vary smoothly with $t$ such that

$$
\chi_{i}(t)^{*}(\psi(t))=\frac{1}{3}\left(v \cdot \varphi_{0}\right)+O\left(r_{i}^{2}\right)
$$

on $V_{i}(t)$ and then extend smoothly to a form $\psi(t) \in C^{\infty}\left(\Lambda^{2} T^{*} T(t)\right)$ such that $d \psi(t)=\left.\varphi\right|_{T(t)}$. Notice that $\psi(0)=\psi$ and $T(0)=T$. Define

$$
H_{2}(\alpha, t)=f_{\alpha}(t)^{*}\left(\left.\psi(t)\right|_{\hat{N}_{\alpha}(t)}\right) .
$$

Then $d\left(H_{2}(\alpha, t)\right)=F_{2}(\alpha, t)$. Moreover, by the same reasoning that showed $H_{1}(\alpha) \in L_{k, \lambda}^{p}$ in the proof of Proposition $7.2, H_{2}(\alpha, t)$ lies in $L_{k, \lambda}^{p}$. Therefore, $F_{2}(\alpha, t)$ lies in $d\left(L_{k, \lambda}^{p}\left(\Lambda^{2} T^{*} \hat{N}\right)\right)$. However, because $F_{2}(\alpha, t) \in L_{k, \lambda-1}^{p}$ $\left(\Lambda^{3} T^{*} \hat{N}\right)$, the proof of Proposition 7.2 implies the result.

We now define the obstruction space.

Definition 7.8. From Propositions 7.3 and 7.7, since $L_{2}$ is a linear map on a finite-dimensional vector space, there exists a finite-dimensional subspace $\mathcal{O}_{2}(N, \lambda)$ of $L_{k, \lambda-1}^{p}\left(\Lambda^{3} T^{*} \hat{N}\right)$, contained in $\mathcal{O}_{1}(N, \lambda)$, such that

$$
\overline{d\left(L_{k+1, \lambda}^{p}\left(\Lambda^{2} T^{*} \hat{N}\right)\right)}=\left(d\left(L_{k+1, \lambda}^{p}\left(\Lambda_{+}^{2} T^{*} \hat{N}\right)\right)+L_{2}\left(T_{0} \hat{\mathcal{T}}\right)\right) \oplus \mathcal{O}_{2}(N, \lambda) .
$$

We define $\mathcal{O}_{2}(N, \lambda)$ to be the obstruction space for Problem 2.

Following the scheme for Problem 1, we let

$$
\begin{aligned}
U_{2} & =L_{k+1, \lambda}^{p}(\hat{U}) \times L_{k+1, \lambda}^{p}\left(\Lambda^{4} T^{*} \hat{N}\right) \times \hat{\mathcal{T}}, \\
X_{2} & =L_{k+1, \lambda}^{p}\left(\Lambda_{+}^{2} T^{*} \hat{N} \oplus \Lambda^{4} T^{*} \hat{N}\right) \oplus T_{0} \hat{\mathcal{T}} \\
Y_{2} & =\frac{\mathcal{O}_{2}(N, \lambda) \subseteq L_{k, \lambda-1}^{p}\left(\Lambda^{3} T^{*} \hat{N}\right) \text { and }}{d\left(L_{k+1, \lambda}^{p}\left(\Lambda^{2} T^{*} \hat{N}\right)\right)} \oplus d^{*}\left(L_{k+1, \lambda}^{p}\left(\Lambda^{4} T^{*} \hat{N}\right)\right) \subseteq L_{k, \lambda-1}^{p}\left(\Lambda^{3} T^{*} \hat{N}\right) .
\end{aligned}
$$

Recall that $\hat{\mathcal{T}} \subseteq \mathbb{R}^{n} \cong T_{0} \hat{\mathcal{T}}$ is open. As for Problem $1, X_{2}, Y_{2}$ and $Z_{2}$ are Banach spaces, $U_{2}$ is an open neighbourhood of $(0,0,0)$ in $X_{2}$ and hence 
$W_{2}=U_{2} \times Y_{2}$ is an open neighbourhood of $((0,0,0), 0)$ in $X_{2} \times Y_{2}$. Define $\mathcal{G}_{2}: W_{2} \rightarrow Z_{2}$ by:

$$
\mathcal{G}_{2}((\alpha, \beta, t), \gamma)=G_{2}(\alpha, \beta, t)+\gamma
$$

From Definition $7.8,\left.d \mathcal{G}_{2}\right|_{((0,0,0), 0)}: X_{2} \times Y_{2} \rightarrow Z_{2}$ acts surjectively as

$$
((\alpha, \beta, t), \gamma) \longmapsto d \alpha+d^{*} \beta+L_{2}(t)+\gamma
$$

Using the fact that the image of $\left.d G_{2}\right|_{(0,0,0)}$ meets $Y_{2}$ at 0 only,

$$
\begin{aligned}
\left.\operatorname{Ker} d \mathcal{G}_{2}\right|_{((0,0,0), 0)} & =\left\{((\alpha, \beta, t), \gamma) \in X_{2} \times Y_{2}: d \alpha+d^{*} \beta+L_{2}(t)+\gamma=0\right\} \\
& \cong\left\{(\alpha, \beta, t) \in X_{2}: d \alpha+d^{*} \beta+L_{2}(t)=0\right\}=\mathcal{I}_{2}(N, \lambda)
\end{aligned}
$$

As for Problem 1, Theorem 4.5 then gives us the following theorem.

Theorem 7.9. Use the notation of Definitions 6.11, 7.6 and 7.8. There exist a smooth manifold $\hat{\mathcal{M}}_{2}(N, \lambda)$, which is an open neighbourhood of $(0,0,0)$ in $\mathcal{I}_{2}(N, \lambda)$, and a smooth map $\pi_{2}: \hat{\mathcal{M}}_{2}(N, \lambda) \rightarrow \mathcal{O}_{2}(N, \lambda)$, with $\pi_{2}(0,0,0)=0$, such that an open neighbourhood of zero in Ker $\pi_{2}$ is homeomorphic to an open neighbourhood of $N$ in $\mathcal{M}_{2}(N, \lambda)$.

We deduce that, if $\mathcal{O}_{2}(N, \lambda)=\{0\}$, the moduli space for Problem 2 is a smooth manifold near $N$ of dimension $\operatorname{dim} \mathcal{I}_{2}(N, \lambda)=\operatorname{dim} \mathcal{I}_{1}(N, \lambda)+\operatorname{dim} \hat{\mathcal{T}}$, which we expect to occur for generic choices of $N$ and the torsion-free $\mathrm{G}_{2}$ structure on $M$. In $\S 9$, we see that if we choose a generic closed $\mathrm{G}_{2}$ structure on $M$, within a certain family, we may drop the genericity assumption on $N$ and still obtain a smooth moduli space.

\subsection{Problem 3}

We presume in this section that the reader is sufficiently familiar with the schemata we have used in $\S 7.1$ and 7.2 to be able to generalize them to Problem 3. This allows us to present a tidier treatment of the problem.

Recall the notation of Definitions 6.20, 6.24 and 6.26. 
Definition 7.10. The infinitesimal deformation space $\mathcal{I}_{3}(N, \lambda)$ for Problem 3 is

$$
\begin{aligned}
\mathcal{I}_{3}(N, \lambda)= & \left\{(\alpha, \beta, t, f) \in L_{k+1, \lambda}^{p}\left(\Lambda_{+}^{2} T^{*} \hat{N} \oplus \Lambda^{4} T^{*} \hat{N}\right) \oplus T_{0} \hat{\mathcal{T}} \oplus T_{0} \hat{\mathcal{F}}: d \alpha\right. \\
& \left.+d^{*} \beta+L_{2}(t)+L_{3}(f)=0\right\} .
\end{aligned}
$$

By Proposition 6.28 and the maximum principle,

$$
\begin{aligned}
\mathcal{I}_{3}(N, \lambda)= & \left\{(\alpha, \beta, t, f) \in C_{\lambda}^{\infty}\left(\Lambda_{+}^{2} T^{*} \hat{N} \oplus \Lambda^{4} T^{*} \hat{N}\right) \oplus T_{0} \hat{\mathcal{T}} \oplus T_{0} \hat{\mathcal{F}}: d \alpha\right. \\
& \left.+d^{*} \beta+L_{2}(t)+L_{3}(f)=0\right\} \\
\cong & \left\{(\alpha, t, f) \in C_{\lambda}^{\infty}\left(\Lambda_{+}^{2} T^{*} \hat{N}\right) \oplus T_{0} \hat{\mathcal{T}} \oplus T_{0} \hat{\mathcal{F}}: d \alpha\right. \\
& \left.+L_{2}(t)+L_{3}(f)=0\right\} .
\end{aligned}
$$

We then have the generalization of Propositions 7.2 and 7.7, which is proved in a similar manner.

Proposition 7.11. In the notation of Definition 6.24,

$$
F_{3}\left(L_{k+1, \lambda}^{p}(\hat{U}) \times \hat{\mathcal{T}} \times \hat{\mathcal{F}}\right) \subseteq \overline{d\left(L_{k+1, \lambda}^{p}\left(\Lambda^{2} T^{*} \hat{N}\right)\right)} \subseteq L_{k, \lambda-1}^{p}\left(\Lambda^{3} T^{*} \hat{N}\right)
$$

This result leads us to define our final obstruction space.

Definition 7.12. Use the notation of Definitions 6.26 and 7.8. From Propositions 7.3 and 7.11 , there exists a finite-dimensional subspace $\mathcal{O}_{3}(N, \lambda)$ of $L_{k, \lambda-1}^{p}\left(\Lambda^{3} T^{*} \hat{N}\right)$, contained in $\mathcal{O}_{2}(N, \lambda)$, such that

$$
\begin{aligned}
& \overline{d(}\left(L_{k+1, \lambda}^{p}\left(\Lambda^{2} T^{*} \hat{N}\right)\right) \\
& \quad=\left(d\left(L_{k+1, \lambda}^{p}\left(\Lambda_{+}^{2} T^{*} \hat{N}\right)\right)+L_{2}\left(T_{0} \hat{\mathcal{T}}\right)+L_{3}\left(T_{0} \hat{\mathcal{F}}\right)\right) \oplus \mathcal{O}_{3}(N, \lambda) .
\end{aligned}
$$

We define $\mathcal{O}_{3}(N, \lambda)$ to be the obstruction space for Problem 3.

The use of the implicit function theorem (Theorem 4.5) in the derivation of Theorems 7.5 and 7.9 can be easily generalized to give the following.

Theorem 7.13. Use the notation of Definitions 6.21, 7.10 and 7.12. There exist a smooth manifold $\hat{\mathcal{M}}_{3}(N, \lambda)$, which is an open neighbourhood of $(0,0$, $0,0)$ in $\mathcal{I}_{3}(N, \lambda)$, and a smooth map $\pi_{3}: \hat{\mathcal{M}}_{3}(N, \lambda) \rightarrow \mathcal{O}_{3}(N, \lambda)$, with $\pi_{3}(0,0$, $0,0)=0$, such that an open neighbourhood of zero in Ker $\pi_{3}$ is homeomorphic to an open neighbourhood of $(N, 0)$ in $\mathcal{M}_{3}(N, \lambda)$. 
Hence, if $\mathcal{O}_{3}(N, \lambda)=\{0\}, \mathcal{M}_{3}(N, \lambda)$ is a smooth manifold near $(N, 0)$ of dimension $\operatorname{dim} \mathcal{I}_{3}(N, \lambda)=\operatorname{dim} \mathcal{I}_{2}(N, \lambda)+\operatorname{dim} \hat{\mathcal{F}}$. Moreover, the projection map $\pi_{\hat{\mathcal{F}}}: \mathcal{M}_{3}(N, \lambda) \rightarrow \hat{\mathcal{F}}$ is smooth near $(N, 0)$. We expect this to occur for generic choices of $N$ and the torsion-free $\mathrm{G}_{2}$ structure on $M$. We show in $\S 9$ that if we work with closed $\mathrm{G}_{2}$ structures, then we may drop our genericity assumptions on $N$ and $(\varphi, g)$ and get a smooth moduli space.

\section{Dimension calculations}

By Theorems 7.5, 7.9 and 7.13, the expected dimension of the moduli space for each problem is the difference between the dimension of the infinitesimal deformation space and the obstruction space. In this section, we first relate the expected dimension of the moduli space for Problem 1 to the index of (5.1) as discussed in $\S 5.2$. We can then deduce an explicit lower bound for the dimension of the moduli space for each of our deformation problems.

Recall the notation introduced at the start of $\S 6$ and $\S 7$. In particular, we fix $\lambda \in(1,2) \backslash \mathcal{D}$, where $\mathcal{D}$ is given by Proposition 5.1, $p>4$ and $k \geq 2$, and let $\rho$ be a radius function on $\hat{N}$ as in Definition 3.2.

Definition 8.1. Define

$$
\mathcal{H}^{m}=\left\{\xi \in L^{2}\left(\Lambda^{m} T^{*} \hat{N}\right): d \xi=d^{*} \xi=0\right\} .
$$

The Hodge star maps $\mathcal{H}^{2}$ into itself, so there is a splitting $\mathcal{H}^{2}=\mathcal{H}_{+}^{2} \oplus \mathcal{H}_{-}^{2}$, where $\mathcal{H}_{ \pm}^{2}=\mathcal{H}^{2} \cap C^{\infty}\left(\Lambda_{ \pm}^{2} T^{*} \hat{N}\right)$.

Let $\mathcal{J}=\jmath\left(H_{\mathrm{cs}}^{2}(\hat{N})\right)$, where $\jmath: H_{\mathrm{cs}}^{2}(\hat{N}) \rightarrow H_{\mathrm{dR}}^{2}(\hat{N})$ is the inclusion map. If $[\alpha],[\beta] \in \mathcal{J}$, there exist compactly supported closed 2 -forms $\xi$ and $\eta$ such that $[\alpha]=\jmath([\xi])$ and $[\beta]=\jmath([\eta])$. We define a product on $\mathcal{J} \times \mathcal{J}$ by

$$
[\alpha] \cup[\beta]=\int_{\hat{N}} \xi \wedge \eta .
$$

Suppose that $\xi^{\prime}$ and $\eta^{\prime}$ are also compactly supported with $[\alpha]=\jmath\left(\left[\xi^{\prime}\right]\right)$ and $[\beta]=\jmath\left(\left[\eta^{\prime}\right]\right)$. Then there exist 1-forms $\chi$ and $\zeta$ such that $\xi-\xi^{\prime}=d \chi$ and $\eta-\eta^{\prime}=d \zeta$. Therefore,

$$
\begin{aligned}
\int_{\hat{N}} \xi^{\prime} \wedge \eta^{\prime} & =\int_{\hat{N}}(\xi-d \chi) \wedge(\eta-d \zeta)=\int_{\hat{N}} \xi \wedge \eta-d \chi \wedge \eta-\xi^{\prime} \wedge d \zeta \\
& =\int_{\hat{N}} \xi \wedge \eta-d(\chi \wedge \eta)-d\left(\xi^{\prime} \wedge \zeta\right)=\int_{\hat{N}} \xi \wedge \eta,
\end{aligned}
$$


as both $\chi \wedge \eta$ and $\xi^{\prime} \wedge \zeta$ have compact support. The product (8.1) on $\mathcal{J} \times \mathcal{J}$ is thus well-defined and is a symmetric topological product with a signature $(a, b)$. By [14, Example 0.16], $\mathcal{H}^{2} \cong \mathcal{J}$ and the isomorphism is given by $\xi \mapsto[\xi]$. Thus, $\operatorname{dim} \mathcal{H}_{+}^{2}=a$ and hence is a topological number.

We use the notation of the next two definitions for the rest of this section.

Definition 8.2. For convenience, we introduce the notation

$$
\left(d_{+}+d^{*}\right)_{\lambda}=d+d^{*}: L_{k+1, \lambda}^{p}\left(\Lambda_{+}^{2} T^{*} \hat{N} \oplus \Lambda^{4} T^{*} \hat{N}\right) \longrightarrow L_{k, \lambda-1}^{p}\left(\Lambda^{3} T^{*} \hat{N}\right) .
$$

By Definition $7.1, \mathcal{I}_{1}(N, \lambda)=\operatorname{Ker}\left(d_{+}+d^{*}\right)_{\lambda}$. Define the adjoint map by

$$
\begin{aligned}
\left(d_{+}^{*}+d\right)_{-3-\lambda}=d_{+}^{*}+d: & L_{l+1,-3-\lambda}^{q}\left(\Lambda^{3} T^{*} \hat{N}\right) \\
& \longrightarrow L_{l,-4-\lambda}^{q}\left(\Lambda_{+}^{2} T^{*} \hat{N} \oplus \Lambda^{4} T^{*} \hat{N}\right)
\end{aligned}
$$

where $q>1$ such that $1 / p+1 / q=1$ and $l \geq k$. (Since we are only concerned with the kernel of this map, which is independent of $l \in \mathbb{N}$ by elliptic regularity, we are free to select $l$.) Then $\operatorname{Coker}\left(d_{+}+d^{*}\right)_{\lambda} \cong \operatorname{Ker}\left(d_{+}^{*}+d\right)_{-3-\lambda}$.

We now study the dimension of the kernel and cokernel of $\left(d_{+}+d^{*}\right)_{\mu}$ to calculate its index. We begin with the kernel.

Proposition 8.3. The kernel of $\left(d_{+}+d^{*}\right)_{-2}$ is isomorphic to $\mathcal{H}_{+}^{2}$. Furthermore, if $\mu>-2$ is such that $(-2, \mu] \cap \mathcal{D}=\emptyset$, $\operatorname{dim} \operatorname{Ker}\left(d_{+}+d^{*}\right)_{\mu}=\operatorname{dim} \mathcal{H}_{+}^{2}$.

Proof. Using (4.1) and the maximum principle,

$$
\begin{aligned}
\mathcal{H}_{+}^{2}= & \left\{\alpha \in L^{2}\left(\Lambda^{2} T^{*} \hat{N}\right) \cap C^{\infty}\left(\Lambda_{+}^{2} T^{*} \hat{N}\right): d \alpha=d^{*} \alpha=0\right\} \\
= & \left\{\alpha \in L_{0,-2}^{2}\left(\Lambda_{+}^{2} T^{*} \hat{N}\right) \cap C^{\infty}\left(\Lambda_{+}^{2} T^{*} \hat{N}\right): d \alpha=0\right\} \\
\cong & \left\{(\alpha, \beta) \in L_{0,-2}^{2}\left(\Lambda_{+}^{2} T^{*} \hat{N} \oplus \Lambda^{4} T^{*} \hat{N}\right): \alpha \in C^{\infty}\left(\Lambda_{+}^{2} T^{*} \hat{N}\right),\right. \\
& \left.d \alpha+d^{*} \beta=0\right\} .
\end{aligned}
$$

This gives the first part of the proposition by elliptic regularity.

If $-2 \notin \mathcal{D},[-2, \mu] \cap \mathcal{D}=\emptyset$ and thus, by Proposition 5.6, $\operatorname{dim} \operatorname{Ker}\left(d_{+}+\right.$ $\left.d^{*}\right)_{\mu}=\operatorname{dim} \operatorname{Ker}\left(d_{+}+d^{*}\right)_{-2}$.

Suppose now that $-2 \in \mathcal{D}$, so that the kernel of $\left(d_{+}+d^{*}\right)_{\nu}$ decreases, or its cokernel increases, as $\nu$ crosses -2 from below by Proposition 5.5. Recall the notation from the start of $\S 6$. Suppose further that $(\alpha, \beta)$ corresponds to a self-dual 2-form and 4 -form on $\hat{N}$ which are subtracted from the kernel. By 
the work in $[15, \S 3$ and $\S 4]$ this occurs if and only if $(\alpha, \beta)$ is asymptotic to an $O\left(r^{-2}\right)$ form $\xi$ on $(0, \epsilon) \times \Sigma_{i} \cong U_{i} \backslash\left\{z_{i}\right\}$, for some $i$, satisfying $\left(d+d^{*}\right) \xi=0$. (The form $\xi$ is determined by an element of $D(-2, i)$, using the notation of Proposition 5.2.) Therefore, $(\alpha, \beta)$ is of order $O\left(\rho^{-2}\right)$ as $\rho \rightarrow 0$ and thus lies in $L^{2}$. We deduce that $(\alpha, \beta) \in \operatorname{Ker}\left(d_{+}+d^{*}\right)_{-2}$, implying that the function $k(\nu)=\operatorname{Ker}\left(d_{+}+d^{*}\right)_{\nu}$ is upper semi-continuous at -2 by Proposition 5.6. The second part of the proposition is thus proved.

Proposition 8.4. If $\mu<-1$ is such that $[\mu,-1) \cap \mathcal{D}=\emptyset$, the cokernel of $\left(d_{+}+d^{*}\right)_{\mu}$ is isomorphic to $H_{\mathrm{dR}}^{1}(\hat{N})$.

Proof. Recall the final part of the proof of Proposition 7.2 where we discussed the map $d+d^{*}$ acting on even forms, and its adjoint map which has kernel $\mathcal{K}$. Since $\mu<-1,-3-\mu>\mu-1$ so $\mathcal{K} \subseteq L_{k, \mu-1}^{p}\left(\Lambda^{\text {odd }} T^{*} \hat{N}\right)$ since $L_{l+1,-3-\mu}^{q} \hookrightarrow$ $L_{k+1, \mu-1}^{p}$ by Theorem 4.4 (using $l \geq k$ ). Moreover, $L_{l+1,-3-\mu}^{q} \hookrightarrow L_{0,-2}^{2}=$ $L^{2}$, which implies that $\mathcal{K}$ is graded and closed under the Hodge star.

Therefore,

$$
L_{k, \mu-1}^{p}\left(\Lambda^{\text {odd }} T^{*} \hat{N}\right)=\overline{\left(d+d^{*}\right)\left(L_{k+1, \mu}^{p}\left(\Lambda^{\text {even }} T^{*} \hat{N}\right)\right)} \oplus \mathcal{K}
$$

If $\gamma \in L_{k, \mu-1}^{p}\left(\Lambda^{3} T^{*} \hat{N}\right)$ then $(-* \gamma, \gamma) \in L_{k, \mu-1}^{p}\left(\Lambda^{\text {odd }} T^{*} \hat{N}\right)$ and hence there exist sequences $\left(\gamma_{n}^{m}\right)$ in $L_{k+1, \mu}^{p}\left(\Lambda^{m} T^{*} \hat{N}\right)$, for $m=0,2,4$, and $\eta \in \mathcal{K}$ such that

$$
(-* \gamma, \gamma)=\eta+\lim _{n \rightarrow \infty}\left(d+d^{*}\right)\left(\gamma_{n}^{0}, \gamma_{n}^{2}, \gamma_{n}^{4}\right)
$$

By applying the Hodge star,

$$
(* \gamma, \gamma)=* \eta+\lim _{n \rightarrow \infty}\left(d-d^{*}\right)\left(* \gamma_{n}^{4}, * \gamma_{n}^{2}, * \gamma_{n}^{0}\right)
$$

Adding the above formulae and averaging gives:

$$
\gamma=\tilde{\eta}+\lim _{n \rightarrow \infty} d\left(\frac{\gamma_{n}^{2}+* \gamma_{n}^{2}}{2}\right)+\lim _{n \rightarrow \infty} d^{*}\left(\frac{\gamma_{n}^{4}-* \gamma_{n}^{0}}{2}\right)
$$

where $\tilde{\eta} \in \mathcal{K}^{3}$ by the properties of $\mathcal{K}$. We deduce that

$$
\begin{aligned}
d\left(L_{k+1, \mu}^{p}\left(\Lambda_{+}^{2} T^{*} \hat{N}\right)\right)+ & d^{*}\left(L_{k+1, \mu}^{p}\left(\Lambda^{4} T^{*} \hat{N}\right)\right) \\
& =\frac{d\left(L_{k+1, \mu}^{p}\left(\Lambda^{2} T^{*} \hat{N}\right)\right)+d^{*}\left(L_{k+1, \mu}^{p}\left(\Lambda^{4} T^{*} \hat{N}\right)\right)}{}
\end{aligned}
$$


because $\mu \notin \mathcal{D}$ implies that the left-hand side is closed. In other words, the image of $\left(d_{+}+d^{*}\right)_{\mu}$ is equal to the closure of the image of

$$
\left(d+d^{*}\right)_{\mu}=d+d^{*}: L_{k+1, \mu}^{p}\left(\Lambda^{2} T^{*} \hat{N} \oplus \Lambda^{4} T^{*} \hat{N}\right) \rightarrow L_{k, \mu-1}^{p}\left(\Lambda^{3} T^{*} \hat{N}\right) .
$$

Thus, Coker $\left(d_{+}+d^{*}\right)_{\mu}$ is isomorphic to the kernel of the adjoint map of (8.2):

$$
\left(d^{*}+d\right)_{-3-\mu}=d^{*}+d: L_{l+1,-3-\mu}^{q}\left(\Lambda^{3} T^{*} \hat{N}\right) \longrightarrow L_{l,-4-\mu}^{q}\left(\Lambda^{2} T^{*} \hat{N} \oplus \Lambda^{4} T^{*} \hat{N}\right) .
$$

Using (4.1) as in the proof of Proposition 8.3, the kernel of $\left(d^{*}+d\right)_{-3-(-1)}$ $=\left(d^{*}+d\right)_{-2}$ is isomorphic to $\mathcal{H}^{3}$. By [14, Example 0.16], $\mathcal{H}^{3} \cong H_{\mathrm{dR}}^{1}(\hat{N})$ and the isomorphism is given by $\gamma \mapsto[* \gamma]$. Since $[\mu,-1) \cap \mathcal{D}=\emptyset$, there are no changes in the cokernel in $[\mu,-1)$ by Proposition 5.6. Moreover, the dimension of the cokernel is lower semi-continuous in $\mu$ at -1 ; this fact can be demonstrated using similar methods to those employed in the proof of Proposition 8.3.

By Proposition 5.3, $(-2,-1] \cap \mathcal{D}=\emptyset$. Therefore, for any $\mu \in(-2,-1]$,

$$
\operatorname{dim} \operatorname{Ker}\left(d_{+}+d^{*}\right)_{\mu}=\operatorname{dim} \mathcal{H}_{+}^{2} \quad \text { and } \operatorname{dim} \operatorname{Coker}\left(d_{+}+d^{*}\right)_{\mu}=b^{1}(\hat{N}),
$$

using Propositions 8.3 and 8.4. Knowing the index of $\left(d_{+}+d^{*}\right)_{\mu}$ for $\mu \in(-2,-1]$, we can calculate it for all growth rates using Theorem 5.5 and hence derive our first estimate of the expected dimension of the moduli space. For the rest of this section, we recall the quantity $d(\mu)$ for $\mu \in \mathcal{D}$, introduced in Definition 5.4, which appears in the statement of Theorem 5.5.

\section{Proposition 8.5.}

$$
\operatorname{ind}\left(d_{+}+d^{*}\right)_{\lambda}=\operatorname{dim} \mathcal{H}_{+}^{2}-b^{1}(\hat{N})-\sum_{\mu \in(-1, \lambda) \cap \mathcal{D}} d(\mu) .
$$

Since the obstruction space $\mathcal{O}_{1}(N, \lambda)$ is a subspace of Coker $\left(d_{+}+d^{*}\right)_{\lambda}$, by relating their dimensions we can improve our estimate.

Proposition 8.6. In the notation of Definition 7.4,

$$
\operatorname{dim} \mathcal{O}_{1}(N, \lambda) \leq \operatorname{dim} \operatorname{Coker}\left(d_{+}+d^{*}\right)_{\lambda}-b^{1}(\hat{N}) .
$$


Proof. From the proof of Proposition 7.2, the closure of the image of (8.2), for $\mu=\lambda$, is characterized as the subspace of $L_{k, \lambda-1}^{p}\left(\Lambda^{3} T^{*} \hat{N}\right)$ which is $L^{2}$ orthogonal to the kernel $\mathcal{L}$ of $\left(d^{*}+d\right)_{-3-\lambda}$ defined by (8.3). Furthermore, as noticed in the proof of Proposition 7.3, Image $\left(d+d^{*}\right)_{\lambda}$ has finite codimension in $L_{k, \lambda-1}^{p}\left(\Lambda^{3} T^{*} \hat{N}\right)$. Therefore, we may choose a finite-dimensional space $\mathcal{C}$ of smooth compactly supported 3 -forms on $\hat{N}$ such that

$$
L_{k, \lambda-1}^{p}\left(\Lambda^{3} T^{*} \hat{N}\right)=\overline{\operatorname{Image}\left(d+d^{*}\right)_{\lambda}} \oplus \mathcal{C}
$$

and so that the product $\mathcal{C} \times \mathcal{L} \rightarrow \mathbb{R}$ given by $(\gamma, \eta) \mapsto\langle\gamma, \eta\rangle_{L^{2}}$ is nondegenerate.

As $\left(d_{+}+d^{*}\right)_{\lambda}$ is Fredholm, its image is the closed subspace of $L_{k, \lambda-1}^{p}$ $\left(\Lambda^{3} T^{*} \hat{N}\right)$ which is $L^{2}$-orthogonal to the kernel $\mathcal{L}^{\prime}$ of $\left(d_{+}^{*}+d\right)_{-3-\lambda}$. Then $\mathcal{L}^{\prime} \supseteq \mathcal{L}$ and $\mathcal{L}$ consists of closed and coclosed 3 -forms, whereas $\mathcal{L}^{\prime}$ consists of 3 -forms $\eta$ such that $d \eta=d_{+}^{*} \eta=0$. Hence, we may choose a subspace $\mathcal{L}^{\prime \prime}$ of $\mathcal{L}^{\prime}$, transverse to $\mathcal{L}$, comprising 3 -forms which are not coclosed and such that $\mathcal{L}^{\prime}=\mathcal{L} \oplus \mathcal{L}^{\prime \prime}$.

The next stage is to extend $\mathcal{C}$ to a space $\mathcal{C}^{\prime}=\mathcal{C} \oplus \mathcal{C}^{\prime \prime}$, where $\mathcal{C}^{\prime \prime}$ consists of smooth exact compactly supported 3 -forms on $\hat{N}$, such that

$$
L_{k, \lambda-1}^{p}\left(\Lambda^{3} T^{*} \hat{N}\right)=\text { Image }\left(d_{+}+d^{*}\right)_{\lambda} \oplus \mathcal{C}^{\prime}
$$

and the product $\mathcal{C}^{\prime \prime} \times \mathcal{L}^{\prime \prime} \rightarrow \mathbb{R}$ given by $(\gamma, \eta) \mapsto\langle\gamma, \eta\rangle_{L^{2}}$ is nondegenerate, which is possible as $\mathcal{L}^{\prime \prime}$ comprises forms which are not coclosed. By construction, $\mathcal{C}^{\prime \prime}$ is a valid choice for $\mathcal{O}_{1}(N, \lambda)$ by the proofs of Propositions 7.2 and 7.3. Therefore,

$$
\operatorname{dim} \mathcal{O}_{1}(N, \lambda)=\operatorname{dim} \mathcal{C}^{\prime}-\operatorname{dim} \mathcal{C}=\operatorname{dim} \operatorname{Coker}\left(d_{+}+d^{*}\right)_{\lambda}-\operatorname{dim} \mathcal{L}
$$

If $\gamma$ lies in the kernel of (8.3) for rate $\mu=-1$, then it also lies in the kernel for $\mu=\lambda$ by Theorem 4.4. Thus, the map from $\mathcal{L}$ to $H_{\mathrm{dR}}^{1}(\hat{N})$ given by $\gamma \mapsto[* \gamma]$ is surjective. This gives the result.

We may now calculate a lower bound for the expected dimension of $\mathcal{M}_{1}(N, \lambda)$ using Propositions 8.5 and 8.6.

Proposition 8.7. Using the notation of Definitions 7.1 and 7.4,

$$
\operatorname{dim} \mathcal{I}_{1}(N, \lambda)-\operatorname{dim} \mathcal{O}_{1}(N, \lambda) \geq \operatorname{dim} \mathcal{H}_{+}^{2}-\sum_{\mu \in(-1, \lambda) \cap \mathcal{D}} d(\mu)
$$


Recalling that the dimension of $\mathcal{T}$ given in Definition 6.12 is $21 s$, we derive analogous results for our other problems.

Proposition 8.8. In the notation of Definitions 6.12, 6.20, 7.6, 7.8, 7.10 and 7.12,

$$
\operatorname{dim} \mathcal{I}_{2}(N, \lambda)-\operatorname{dim} \mathcal{O}_{2}(N, \lambda) \geq \operatorname{dim} \mathcal{H}_{+}^{2}+21 s-\operatorname{dim} \mathrm{H}-\sum_{\mu \in(-1, \lambda) \cap \mathcal{D}} d(\mu) .
$$

and

$$
\begin{aligned}
\operatorname{dim} \mathcal{I}_{3}(N, \lambda)-\operatorname{dim} \mathcal{O}_{3}(N, \lambda) \geq & \operatorname{dim} \mathcal{H}_{+}^{2}+21 s-\operatorname{dim} \mathrm{H} \\
& +\operatorname{dim} \hat{\mathcal{F}}-\sum_{\mu \in(-1, \lambda) \cap \mathcal{D}} d(\mu) .
\end{aligned}
$$

We note that Propositions 5.3, 8.4, 8.6 and 8.7 imply the following bound on $\operatorname{dim} \mathcal{O}_{1}(N, \lambda)$.

Proposition 8.9. Using the notation of Definition 7.4,

$$
\operatorname{dim} \mathcal{O}_{1}(N, \lambda) \leq \sum_{\mu \in(-1, \lambda) \cap \mathcal{D}} d(\mu)
$$

In Problem 2, we remove the obstructions corresponding to translations of the singularities and $\mathrm{G}_{2}$ transformations of the tangent cones. These obstructions occur, respectively, at rates 0 and 1 . Hence, $d(0) \geq 7 s, d(1) \geq$ $14 s-\operatorname{dim} \mathrm{H}$ and we have the following stronger bound on the dimension of $\mathcal{O}_{2}(N, \lambda)$.

Proposition 8.10. In the notation of Definitions 6.12 and 7.8,

$$
\operatorname{dim} \mathcal{O}_{2}(N, \lambda) \leq-21 s+\operatorname{dim} \mathrm{H}+\sum_{\mu \in(-1, \lambda) \cap \mathcal{D}} d(\mu)
$$

\section{9. $\varphi$-Closed 7-manifolds}

For our deformation problems, we have assumed that $N$ is a CS coassociative 4-fold in a $\mathrm{G}_{2}$ manifold $(M, \varphi, g)$; that is, $\varphi$ satisfies $d \varphi=d^{*} \varphi=0$. However, the results of McLean [17] we have used, which are based upon the linearization of the map we denoted $F_{1}$ in Definition 6.5 , still hold if this condition on $\varphi$ is relaxed to just $d \varphi=0$. Thus, our deformation theory results hold if $(M, \varphi, g)$ is a $\varphi$-closed 7-manifold in the sense of Definition 2.5. 
Remark. The effect of $* \varphi$ not being closed on $M$ means that coassociative 4 -folds in $M$ are no longer necessarily volume minimizing in their homology class.

The use of $\varphi$-closed 7 -manifolds $M$ is that closed $\mathrm{G}_{2}$ structures occur in infinite-dimensional families, since the set of closed definite 3-forms on $M$, given in Definition 2.3, is open. Thus, if $m=\operatorname{dim} \mathcal{O}_{1}(N, \lambda)$, we show that we can always choose a family $\mathcal{F}$ of closed $\mathrm{G}_{2}$ structures on $M$, parameterized by $\hat{\mathcal{F}} \subseteq \mathbb{R}^{m}$, such that $\mathcal{O}_{3}(N, \lambda)=\{0\}$. Hence, by Theorem $7.13, \mathcal{M}_{3}(N, \lambda)$ is a smooth manifold and the projection $\pi_{\hat{\mathcal{F}}}: \mathcal{M}_{3}(N, \lambda) \rightarrow \hat{\mathcal{F}}$ is a smooth map near $(N, 0)$.

Sard's theorem [12, p. 173] states that, if $h: X \rightarrow Y$ is a smooth map between finite-dimensional manifolds, the set of $y \in Y$ with some $x \in h^{-1}(y)$ such that $\left.d h\right|_{x}: T_{x} X \rightarrow T_{y} Y$ is not surjective is of measure zero in $Y$. Therefore, $h^{-1}(y)$ is a submanifold of $X$ for almost all $y \in Y$.

Thus, by Sard's theorem, $\pi_{\hat{\mathcal{F}}}^{-1}(f)$ is a smooth manifold near $(N, f)$ for almost all $f \in \hat{\mathcal{F}}$. As observed in Definition $6.21, \pi_{\hat{\mathcal{F}}}^{-1}(f)$ corresponds to the moduli space of deformations for Problem 2 defined using the $\mathrm{G}_{2}$ structure $\left(\varphi^{f}, g^{f}\right)$. We deduce that, for any given $N$, a generic perturbation of the closed $\mathrm{G}_{2}$ structure within $\mathcal{F}$ ensures that $\mathcal{M}_{2}(N, \lambda)$ is smooth near $N$.

We thus conclude with a generic smoothness result for the CS coassociative deformation theory in $\varphi$-closed 7 -manifolds. This is similar to [7, Theorem 9.1] concerning CS special Lagrangian deformations in almost Calabi-Yau manifolds.

Theorem 9.1. Let $(M, \varphi, g)$ be a $\varphi$-closed 7-manifold and let $N$ be a $C S$ coassociative 4-fold in $M$ at $s$ points with rate $\lambda \in(1,2) \backslash \mathcal{D}$, where $\mathcal{D}$ is given in Proposition 5.2. Use the notation of Definitions 5.4, 6.12, 7.4, 7.12 and 8.1. Let $m=\operatorname{dim} \mathcal{O}_{1}(N, \lambda)$ and let $\hat{\mathcal{F}}$ be a small open ball about 0 in $\mathbb{R}^{m}$.

There exists a smooth family $\mathcal{F}=\left\{\left(\varphi^{f}, g^{f}\right): f \in \hat{\mathcal{F}}\right\}$ of closed $\mathrm{G}_{2}$ structures on $M$ such that $\mathcal{O}_{3}(N, \lambda)=\{0\}$. Hence, the moduli space of deformations for Problem 3 is a smooth manifold near $(N, 0)$ with dimension

$$
\operatorname{dim} \mathcal{M}_{3}(N, \lambda) \geq \operatorname{dim} \mathcal{H}_{+}^{2}+21 s-\operatorname{dim} \mathrm{H}+\operatorname{dim} \mathcal{O}_{1}(N, \lambda)-\sum_{\mu \in(-1, \lambda) \cap \mathcal{D}} d(\mu) .
$$

Moreover, for generic $f \in \hat{\mathcal{F}}$, the moduli space of deformations in $\left(M, \varphi^{f}, g^{f}\right)$ for Problem 2 is a smooth manifold near $N$ with dimension

$$
\operatorname{dim} \mathcal{M}_{2}(N, \lambda) \geq \operatorname{dim} \mathcal{H}_{+}^{2}+21 s-\operatorname{dim} \mathrm{H}-\sum_{\mu \in(-1, \lambda) \cap \mathcal{D}} d(\mu)
$$


Proof. Use the notation in the proof of Proposition 8.6. Recall that we have a subspace $\mathcal{L}^{\prime \prime}$ of $L_{l+1,-3-\lambda}^{q}\left(\Lambda^{3} T^{*} \hat{N}\right)$ consisting of forms $\eta$ such that $d \eta=d_{+}^{*} \eta=0$ but $d^{*} \eta \neq 0$. Moreover, $\mathcal{O}_{1}(N, \lambda)$ can be chosen to be a space of smooth compactly supported exact 3-forms $\gamma$ such that if $\langle\gamma, \eta\rangle_{L^{2}}=0$ for all $\eta \in \mathcal{L}^{\prime \prime} \backslash\{0\}$, then $\gamma=0$. Therefore, $\mathcal{L}^{\prime \prime} \cong\left(\mathcal{O}_{1}(N, \lambda)\right)^{*}$ and hence has dimension $m$.

Let $\left\{\eta_{1}, \ldots, \eta_{m}\right\}$ be a basis for $\mathcal{L}^{\prime \prime}$ and choose a basis $\left\{d v_{1}, \ldots, d v_{m}\right\}$ for $\mathcal{O}_{1}(N, \lambda)$, where $v_{j}$ is a smooth compactly supported 2 -form for all $j$, such that $\left\langle d v_{i}, \eta_{j}\right\rangle_{L^{2}}=\delta_{i j}$. This is possible because the $L^{2}$ product on $\mathcal{O}_{1}(N, \lambda) \times$ $\mathcal{L}^{\prime \prime}$ is nondegenerate. For $f=\left(f_{1}, \ldots, f_{m}\right) \in \mathbb{R}^{m}$ define $v_{f}=\sum_{j=1}^{m} f_{j} v_{j}$.

Using the notation of Proposition 6.19, define $\left(\varphi^{f}, g^{f}\right)$, for $f \in \hat{\mathcal{F}}$, to be a closed $\mathrm{G}_{2}$ structure on $M$ such that $\Xi\left(\left[\left.\varphi^{f}\right|_{T}\right]\right)=0$ in $H_{\mathrm{cs}}^{3}(\hat{N})$ and $\left.\varphi^{f}\right|_{\hat{N}}=d v_{f}$.

Recall from Definition 6.24 that we have a linear map $L_{3}: T_{0} \hat{\mathcal{F}} \cong \mathbb{R}^{m} \rightarrow$ $L_{k, \lambda-1}^{p}\left(\Lambda^{3} T^{*} \hat{N}\right)$ arising from $\left.d F_{3}\right|_{(0,0,0)}$. By construction, $L_{3}(f)=d v_{f}$ for $f \in \mathbb{R}^{m}$ and hence $L_{3}$ maps onto $\mathcal{O}_{1}(N, \lambda)$. Proposition 7.3 and Definition 7.12 imply that $\mathcal{O}_{3}(N, \lambda)=\{0\}$ as required.

The latter parts of the theorem now follow directly from the discussion preceding it and Proposition 8.8.

\section{Acknowledgments}

Many thanks go to Dominic Joyce for his enormous help with this project. I would also like to thank Alexei Kovalev for useful comments and suggestions and EPSRC for providing the funding for this research.

\section{References}

[1] R. Bartnik, The mass of an asymptotically flat manifold, Comm. Pure Appl. Math. 39, (1986), 661-693.

[2] R.L. Bryant, Some remarks on $\mathrm{G}_{2}$-structures, in Proceedings of Gökova Geometry - Topology Conference 2005, eds. S. Akbulut, T. Önder and R.J. Stern, International Press, Boston, 2006.

[3] R. Harvey and H.B. Lawson, Calibrated geometries, Acta Math. 148 (1982), 47-152.

[4] H. Federer, Geometric measure theory, Grundlehren Math. Wiss., 153, Springer-Verlag, Berlin, 1969. 
[5] D.D. Joyce, Compact manifolds with special holonomy, OUP, Oxford, 2000.

[6] D.D. Joyce, Special Lagrangian submanifolds with isolated conical singularities. I. Regularity, Ann. Global Ann. Geom. 25 (2004), 201-251.

[7] D.D. Joyce, Special Lagrangian submanifolds with isolated conical singularities. II. Moduli spaces, Ann. Global Ann. Geom. 25 (2004), 301-352.

[8] D.D. Joyce, Special Lagrangian submanifolds with isolated conical singularities. III. Desingularization, the unobstructed case, Ann. Global Ann. Geom. 26 (2004), 1-58.

[9] D.D. Joyce, Special Lagrangian submanifolds with isolated conical singularities. IV. Desingularization, obstructions and families, Ann. Global Ann. Geom. 26 (2004), 117-174.

[10] D.D. Joyce, Special Lagrangian submanifolds with isolated conical singularities. V. Survey and applications, J. Differential Geom. 63 (2003), 299-347.

[11] A. Kovalev, Coassociative K3 fibrations of compact $\mathrm{G}_{2}$-manifolds, Preprint, 2005, http://www.arxiv.org/math.DG/0511150.

[12] S. Lang, Differential manifolds, Addison-Wesley, Reading, Massachusetts, 1972.

[13] S. Lang, Real analysis, 2nd edn, Addison-Wesley, Reading, Massachusetts, 1983.

[14] R.B. Lockhart, Fredholm, Hodge and Liouville theorems on noncompact manifolds, Trans. Amer. Math. Soc. 301 (1987), 1-35.

[15] R.B. Lockhart and R.C. McOwen, Elliptic differential operators on noncompact manifolds, Ann. Sci. Norm. Super. Pisa Cl. Sci. 12 (1985), 409-447.

[16] J. Lotay, Deformation theory of asymptotically conical coassociative 4-folds, Preprint, 2004, http://www.arxiv.org/math.DG/0411116.

[17] R.C. McLean, Deformations of calibrated submanifolds, Comm. Anal. Geom. 6 (1998), 705-747.

[18] S.P. Marshall, Deformations of special Lagrangian submanifolds, DPhil thesis, Oxford University, 2002. 
[19] V.G. Maz'ya and B. Plamenevskij, Elliptic boundary value problems, Amer. Math. Soc. Transl. 123 (1984), 1-56.

[20] C.B. Morrey, Multiple integrals in the calculus of variations, Grundlehren Series, 130, Springer-Verlag, Berlin, 1966.

[21] S. Salamon, Riemannian geometry and holonomy groups, Pitman Research Notes in Mathematics, 201, Longman, Harlow, 1989.

University College

High Street

OXFORD, OX1 4BH

$\mathrm{UK}$

E-mail address: lotayj@maths.ox.ac.uk 